\section{SANDIA REPORT}

SAND98-0270 • UC-523

Unlimited Release

Printed February 1998
RECPIVED

FCD 231998

\title{
Development of Explosive Event Scale Model Testing Capability at Sandia's Large Scale Centrifuge Facility
}

T. K. Blanchat, N. T. Davie, J. J. Calderone, T. C. Togami, D. S. Preece, J. R. Weatherby, R. A. Benham

Sandia National Laboratories

Albuquerque, New Mexico 87185 and Livermore, California 94550

Sandia is a multiprogram laboratory operated by Sandia Corporation, a Lockheed Martin Company, for the Unilied States Department of

Energy under Contract DE-AC04-94AL85000.

Approved for public release; further dissemination unlimited.

\section{Sandia National Laboratories}

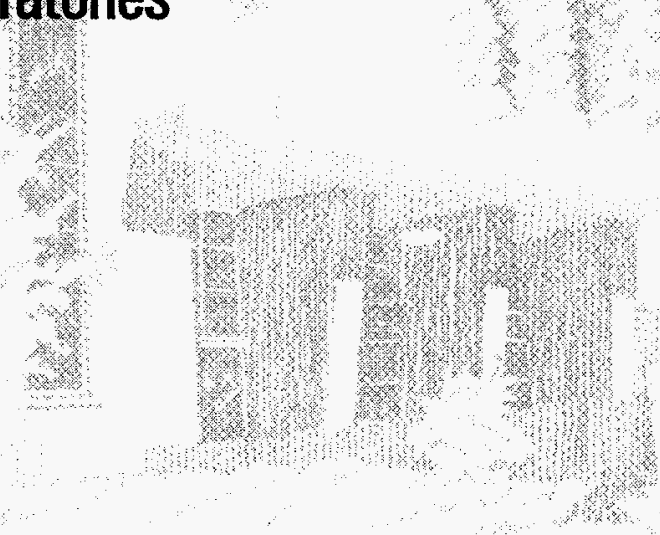


Issued by Sandia National Laboratories, operated for the United States Department of Energy by Sandia Corporation.

NOTICE: This report was prepared as an account of work sponsored by an agency of the United States Government. Neither the United States Government nor any agency thereof, nor any of their employees, nor any of their contractors, subcontractors, or their employees, makes any warranty, express or implied, or assumes any legal liability or responsibility for the accuracy, completeness, or usefulness of any information, apparatus, product, or process disclosed, or represents that its use would not infringe privately owned rights. Reference herein to any specific commercial product, process, or service by trade name, trademark, manufacturer, or otherwise, does not necessarily constitute or imply its endorsement, recommendation, or favoring by the United States Government, any agency thereof, or any of their contractors or subcontractors. The views and opinions expressed herein do not necessarily state or reflect those of the United States Government, any agency thereof, or any of their contractors.

Printed in the United States of America. This report has been reproduced directly from the best available copy.

Available to DOE and DOE contractors from

Office of Scientific and Technical Information

P.O. Box 62

Oak Ridge, TN 37831

Prices available from (615) 576-8401, FTS 626-8401

Available to the public from

National Technical Information Service

U.S. Department of Commerce

5285 Port Royal Rd

Springfield, VA 22161

NTIS price codes

Printed copy: A04

Microfiche copy: A01

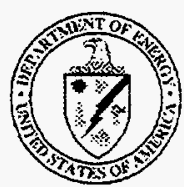




\section{DISCLAIMER}

Portions of this document may be illegible electronic image products. Images are produced from the best available original document. 
SAND98-0270

Distribution

Unlimited Release

Category UC-523

Printed February 1998

\title{
Development of Explosive Event Scale Model Testing Capability at Sandia's Large Scale Centrifuge Facility
}

T. K. Blanchat, N. T. Davie*, J. J. Calderone*, T. C. Togami*

D. S. Preece**, J. R. Weatherby***, R. A. Benham****

\author{
Reactor Safety Experiments Department \\ Albuquerque Full-Scale Experimental Complex Department* \\ Geomechanics Department** \\ Computational Physics R\&D Department*** \\ Explosive Components Department**** \\ Sandia National Laboratories \\ P. O. Box 5800 \\ Albuquerque, NM 87185-1139
}

\begin{abstract}
Geotechnical structures such as underground bunkers, tunnels, and building foundations are subjected to stress fields produced by the gravity load on the structure and/or any overlying strata. These stress fields may be reproduced on a scaled model of the structure by proportionally increasing the gravity field through the use of a centrifuge. This technology can then be used to assess the vulnerability of various geotechnical structures to explosive loading. Applications of this technology include assessing the effectiveness of earth penetrating weapons, evaluating the vulnerability of various structures, counter-terrorism, and model validation. This document describes the development of expertise in scale model explosive testing on geotechnical structures using Sandia's large scale centrifuge facility. This study focused on buried structures such as hardened storage bunkers or tunnels. Data from this study was used to evaluate the predictive capabilities of existing hydrocodes and structural dynamics codes developed at Sandia National Laboratories (such as Pronto/SPH, Pronto/CTH, and ALEGRA).
\end{abstract}




\section{Acknowledgments}

The authors would like to thank J. R. Garcia for his oversight and guidance of the program. The authors express their gratitude to Glen Perdue (centrifuge operational procedures and test support), Dennis Floyd (centrifuge operational procedures, test support, and console operator), Jerry Stoffleth (fire sets and other associated explosive hardware), Dave Wackerbarth (fabrication of the explosive system), Mike Baca (explosive handling, arming and firing), and Jim Dotts (Sandia safety consultant), Ben Duggins (instrumentation consultation and data reduction), and Western Diversified Inc. (skilled labor support for soil collection and experiment assembly).

This work was supported by the United States Department of Energy under Contract No. DE-AC04-94AL85000. Sandia is a multiprogram laboratory operated by Sandia Corporation, a Lockheed Martin Company, for the United States Department of Energy. The support of DOE/Sandia Laboratory-Directed Research and Development (LDRD) funds is gratefully acknowledged. 


\section{Contents}

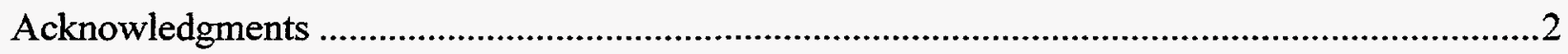

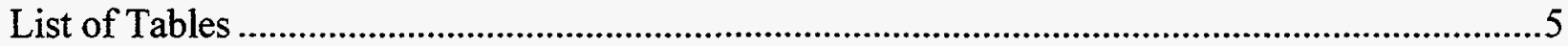

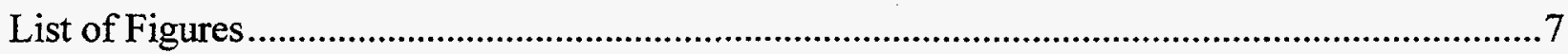

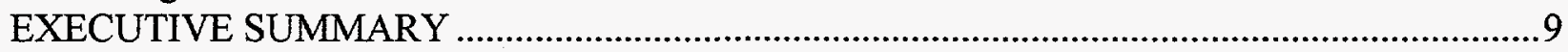

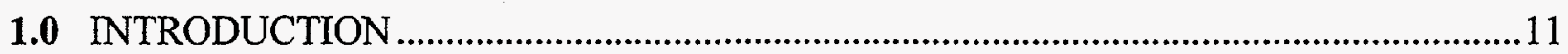

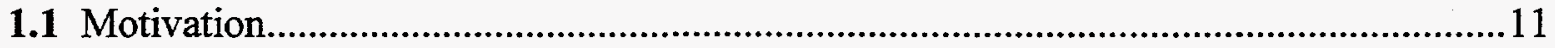

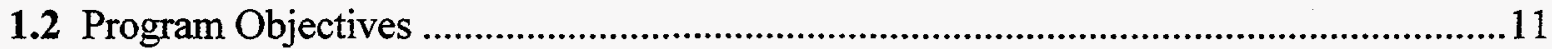

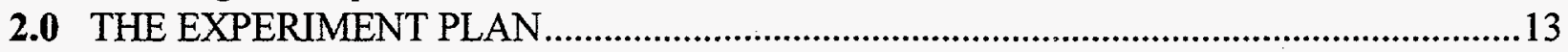

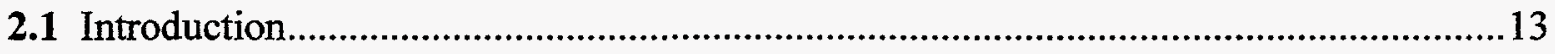

2.1 Description of the Large Scale Centrifuge Facility ....................................................15

2.1 Centrifuge Scaling Relations .................................................................................16

3.0 EXPERIMENT DESIGN USING COMPUTER SIMULATIONS .................................19

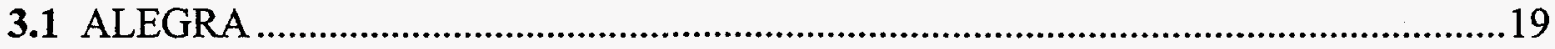

3.2 Preliminary Calculations...................................................................................19

3.3 Detailed Two-Dimensional Simulations ..................................................................21

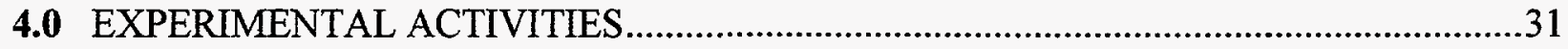

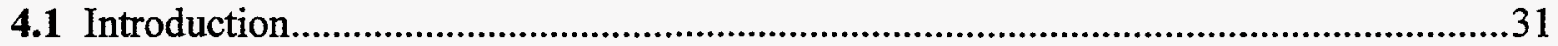

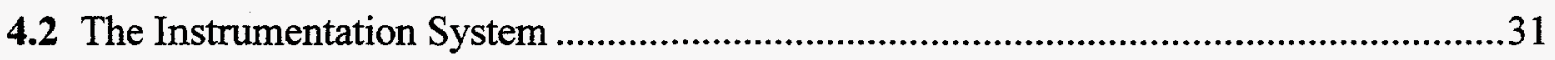

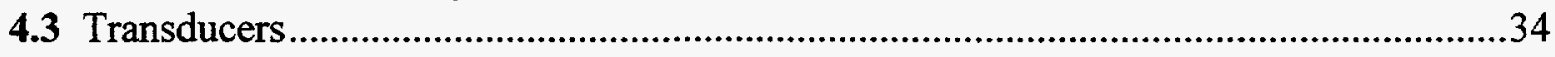

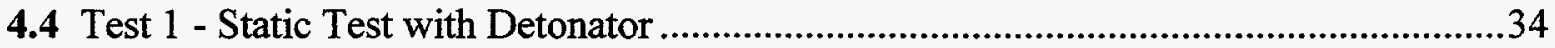

4.5 Test 2 - Static Test in Load Frame with 3 Gram Charge ...........................................36

4.6 Test 3 - $100 \mathrm{G}$ Centrifuge Test with 3 Gram Charge...............................................38

4.7 Experimental Results and Analytical Model Predictions ...........................................40

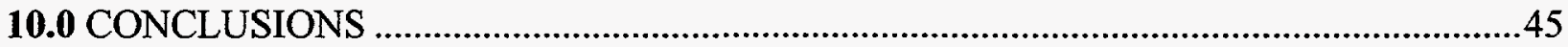

Appendix A THE SCALED TUNNEL MODEL HARDWARE .............................................47

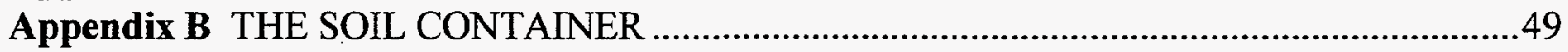

Appendix C MANUFACTURED SOIL AND EXPERIMENT ASSEMBLY .......................53

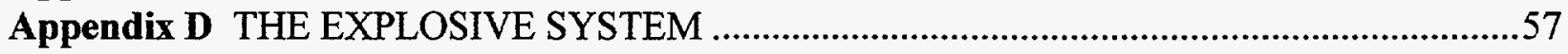

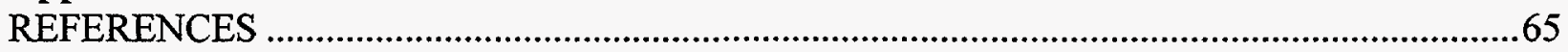

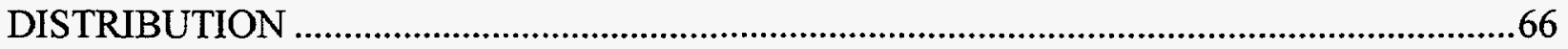


This page intentionally left blank. 


\section{Contents (continued)}

\section{List of Tables}

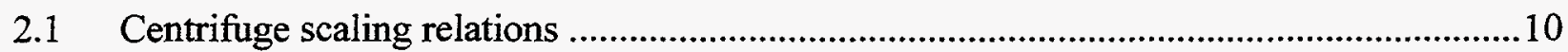

3.1 JWL equation-of-state parameters for PBX-9407 ...........................................................13

3.2 Soil and crushable foam constitutive model parameters for engineered soil.......................13

3.3 Pressure versus volumetric strain for engineered soil.........................................................13

3.4 Elastic-perfectly-plastic constitutive parameters for aluminum tube .....................................14

3.5 Ideal gas parameters for air ...................................................................................... 14

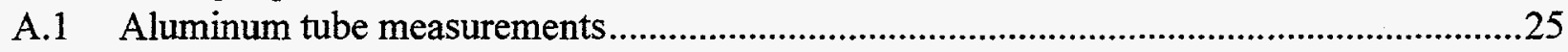

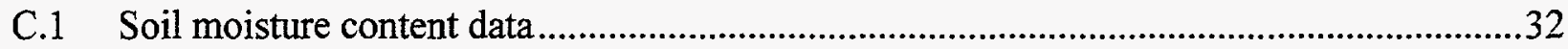





\section{Contents (continued)}

\section{List of Figures}

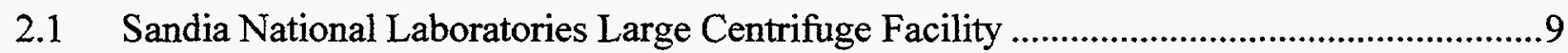

2.2 The geotechnical swing-arm assembly .....................................................................

3.1 Pseudo one-dimensional ALE model used to design the decoupled explosive tunnel

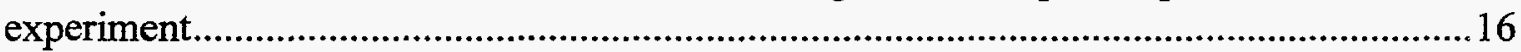

3.2 Two-dimensional ALE model used to predict the response of the model to a decoupled explosive in a tunnel ............................................................................................16

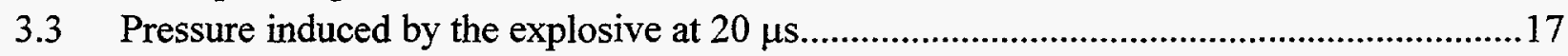

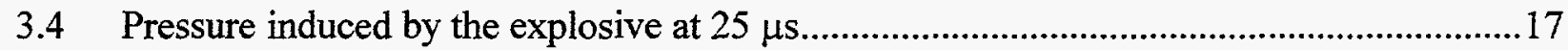

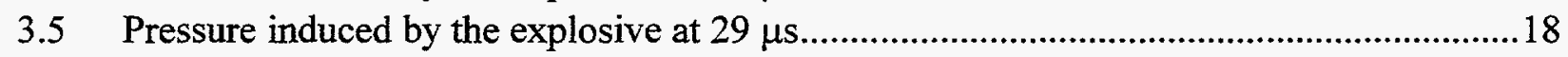

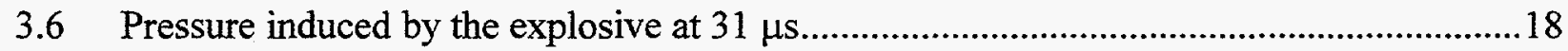

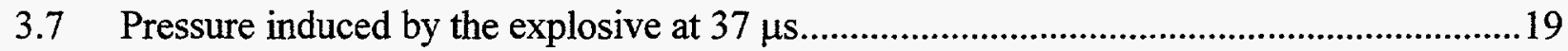

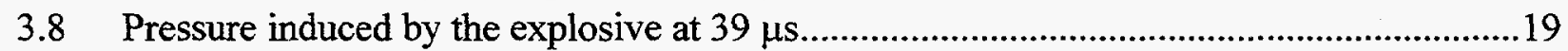

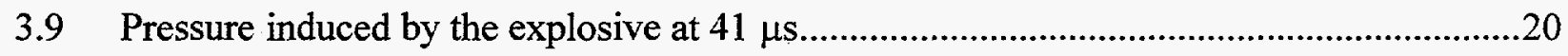

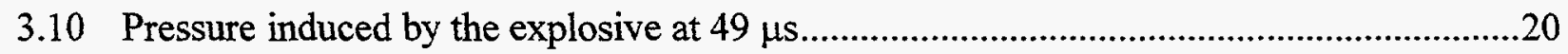

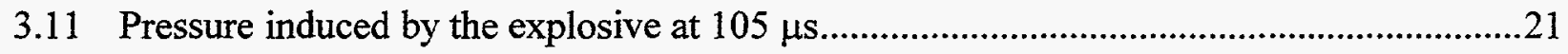

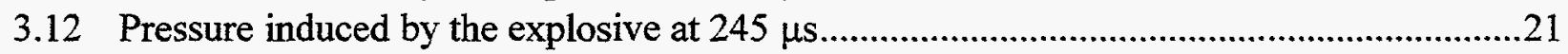

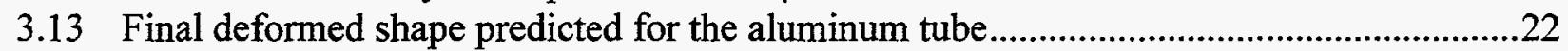

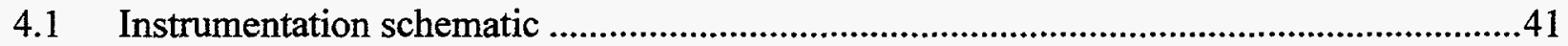

4.2 The instrumentation rack installed on the center of the centrifuge ........................................42

4.3 The technique used to accommodate large tube movement................................................44

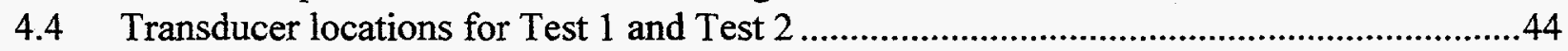

4.5 The instrumented assembly placed in a load frame ...........................................................45

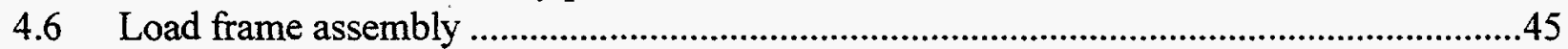

4.7 The center of the tube expanded by the action of the explosive charge..............................46

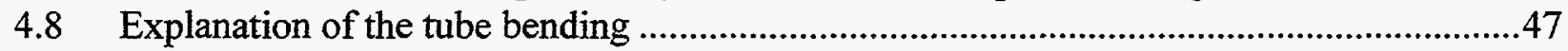

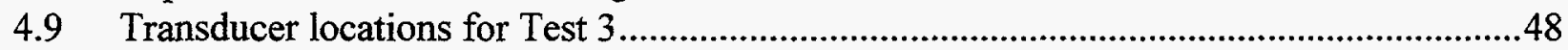

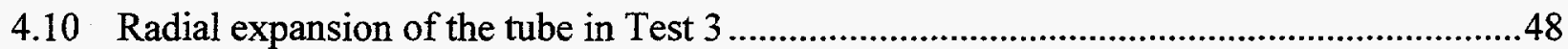

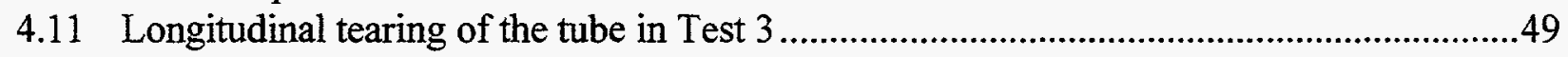

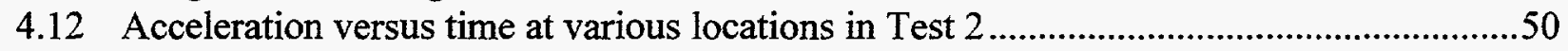

4.13 Hoop strain versus time at various locations in Test 2 ........................................................51

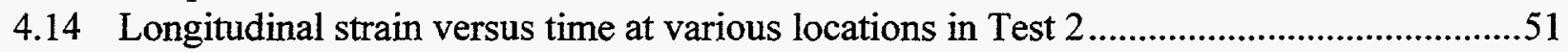

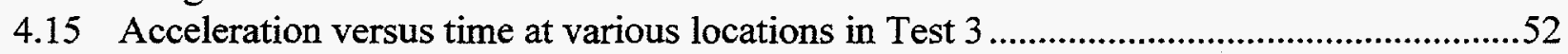

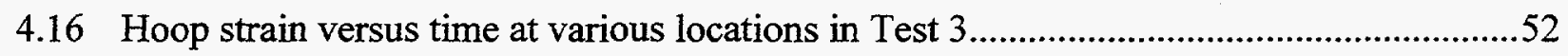

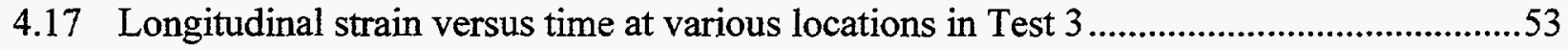

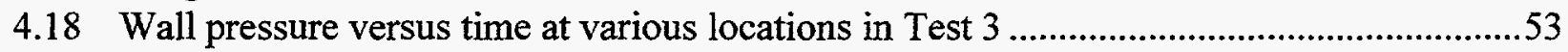

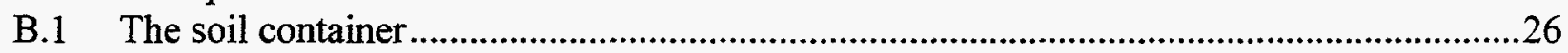

B.2 Inserting an explosive charge into an assembled test fixture ..........................................28

C.1 The Albuquerque sand/clay soil mixture ……................................................................29

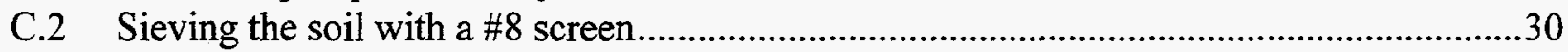

C.3 Partial filling the drums with the sieved soil................................................................30 


\section{Contents (concluded)}

\section{List of Figures}

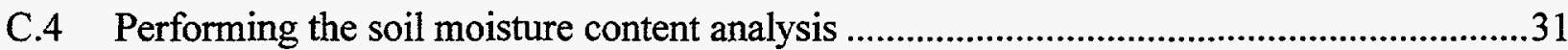

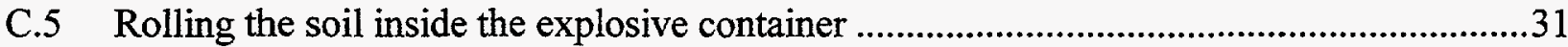

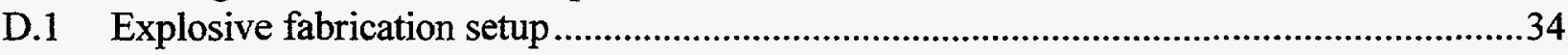

D.2 Sketch of the explosive system .......................................................................................3

D.3 Explosive system minus the detonator, mounted on the support pedestal ...........................35

D.4 Tube with the thin film carbon gage in place (electrical tape placed over the gage) ...........35

D.5 Explosive charge on the pedestal with the rubber covered tube ............................................36

D.6 Test setup in test chamber, pre-test ..................................................................................37

D.7 Test setup in test chamber, post-test ................................................................................38

D.8 Tube, showing the bulge after the test ............................................................................3

D.9 Data from pretest heating superimposed on the explosive test data .....................................39

D.10 Corrected data from the explosive test along with the integrated data ................................39 


\section{Executive Summary}

This report summarizes the work performed under the Sandia Laboratory-Directed Research and Development (LDRD) project "Development of Explosive Event Scale Model Testing Capability at Sandia."

This effort has demonstrated a state-of-the-art test capability using both Sandia's Large Centrifuge Facility and analytical expertise to conduct scale model experiments that assess the explosive vulnerability of various structures. The scaled model approach of the centrifuge provided an accurate simulation to validate the models and codes without the expense and risks incurred in full-scale explosive testing. Expertise in high-speed data acquisition of an explosive event in steady-state high-G fields, scaling of structures and explosives, and soil preparation was acquired. This study also provided data to evaluate the predictive capability of Sandia's phenomenological models and computer codes. The successful completion of this study has given Sandia a unique capability that can be applied to critical, emerging threat needs in the national interests. 



\subsection{INTRODUCTION}

\subsection{Motivation}

Centrifuges have a 60 year history for the testing of scaled geotechnical structures. Sandia National Laboratories operates two centrifuges, one of which is among the largest dynamic load capacity in the world. Sandia's Large Centrifuge Facility has been used to study topics such as soil permeability, slope stability, and more. This study provided an opportunity to develop expertise in the testing of explosive loading on scaled geotechnical structures at Sandia, thus enhancing the unique capabilities of the Large Centrifuge Facility.

The hypothesis for this study was to verify that gravity scaling laws are valid for explosive events in buried structures and to establish the capability to assess the vulnerability of various geotechnical structures to explosive loading. In particular, methods of combining explosive loading to the steady-state centrifuge acceleration field were investigated. The data gathered from this study were used to evaluate the predictive capabilities of Sandia's phenomenological models and computer codes. The scaled model approach of the centrifuge provided an accurate simulation to validate the models and codes without the expense and risks incurred in full-scale explosive testing.

\subsection{Program Objectives}

This study was a marriage of the large scale test facilities and the predictive capabilities of computer simulation techniques at Sandia. A Sandia team was assembled to develop a suitable scaled test configuration that could be used to validate existing computer codes and constitutive models that predict the response of full-size earth structures to explosive events. This team included expertise in explosive testing and analysis, geomechanics, material and structural mechanics, and full scale experiments. For test development, emphasis was placed on problems which complement Sandia's mission. Based on predictions generated by the hydrocodes and structural dynamics codes (Pronto/SPH, Pronto/CTH and ALEGRA), an appropriate class of geotechnical structures was identified for modeling and testing. Focus was placed on ground shock effects on underground structures such as tunnels or covered bunkers. A scaled underground tunnel model was built using existing scaling laws. In order to help develop this testing capability, several tests on the model were performed. These tests included explosive tests with the model not subjected to the centrifuge g-field. The final test on the scaled geotechnical structure included both centrifugal loading and explosive loading. Measured data was compared with the predicted results of existing hydrocodes and structural dynamics codes. 



\subsection{THE EXPERIMENT PLAN}

\subsection{Introduction}

The LDRD project proposed gravity scaling of an explosive event in a steady-state acceleration environment available through centrifuge testing. Many different types of explosive events amenable to gravity scaling were available including: 1) cratering, 2) a scale model building with explosively induced, gravity driven progressive collapse, and 3) tunnels in rock or soil subjected to decoupled explosive loading (Mosaic 1979). After weighing time, budget, and complexity of effort constraints, it was decided to focus on a decoupled explosion in a tunnel residing in an engineered soil. This configuration was chosen because length scales directly with gravity on underground structures in regards to stress, deformation, and the physical dimension of the structure.

Based on extensive computer simulation (Section 3), a 1/100 scale model representing approximately a 13-foot diameter tunnel buried about 100 feet in soil was chosen to demonstrate the technique. A 3 gram explosive charge representing about 6600 pounds of TNT (scaled due to the $100 \mathrm{G}$ centrifuge loading) was fired in the center of the tunnel. The experiment configuration consisted of a $2 \mathrm{ft} \times 2 \mathrm{ft} \times 2 \mathrm{ft}$ aluminum container containing an engineered soil. A small aluminum pipe (1.5-inch OD) was placed horizontally in the center of the container. The pipe ends protruded through slots in the soil container walls.

\section{Scaled Centrifuge Model at 100 G's}

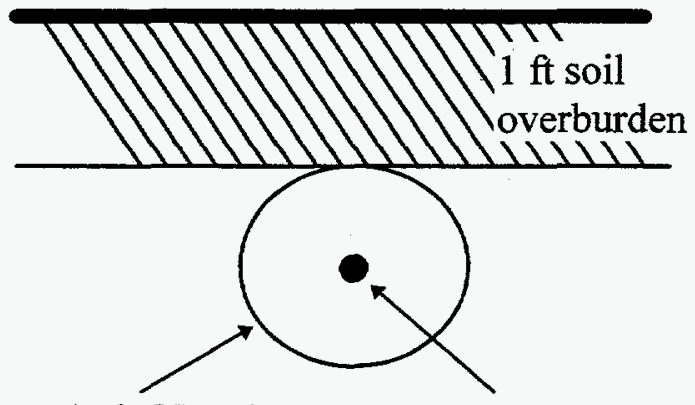

$1 / 8 \mathrm{ft} \mathrm{OD}$ tube (1.5 in)
$6.1 \times 10^{-3} \mathrm{lb}$ explosive $(2.75 \mathrm{~g})$
Full Size Representation at 1 G

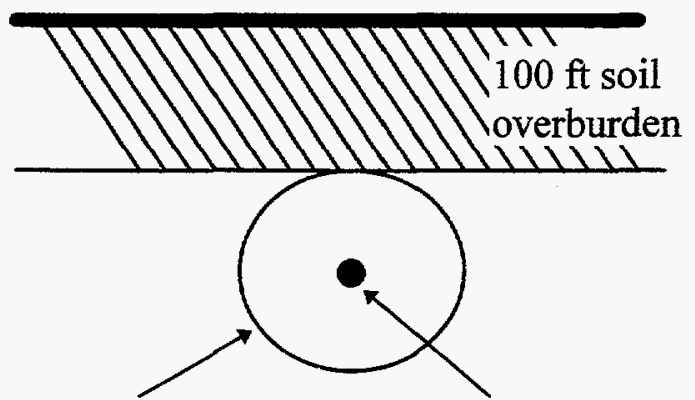

$12.5 \mathrm{ft} \mathrm{OD}$ tunnel

(150 in)
$6.1 \times 10^{3} \mathrm{lb}$ explosive

(3 tons of TNT)

The 3 gram explosive charge underwent a proof-of-design test inside a sample section of the aluminum tube (Appendix A). Three explosive tests were performed at the Large Centrifuge Facility (LCF). The first test was a static fire test of the experiment configuration (loaded with engineered soil and partially instrumented) using only a RP-2 detonator. The second test was a static fire test in a load frame of the same experiment configuration using the 3 gram charge. The last test was a dynamic test (on the centrifuge at $100 \mathrm{G}$ ) using a newly configured experiment loaded with a new aluminum pipe, engineered soil, complete instrumentation, and a 3 gram charge. 
A geotechnical fixture (Appendix B) was designed to withstand the maximum expected centrifuge loading with $\mathrm{a}>2$ safety factor. This fixture underwent a proof pressure test in a static load frame prior to being subjected to the centrifuge loading. A well-characterized engineered soil was fabricated (Appendix C). The 3 gram explosive charge was designed and fabricated (Appendix D) based on extensive code modeling. Based on the code results, accelerometers, strain gages, and pressure gages were installed at specific locations identified to provide code comparison and validation. The primary data acquisition system (DAQ) consisted of Tektronix digitizing oscilloscopes along with appropriate signal conditioning. The following lists the key tasks in order of performance:

1) Computer modeling and simulation to provide experiment design parameters.

2) A robust test fixture was designed to withstand the hydraulic load of the soil in a $100 \mathrm{G}$ acceleration field with a superimposed impulsive load of a 3 gram explosive charge, with a safety factor $>2$.

3) A load frame with hydraulic ram capable of compressing the soil to 60 psi (the maximum pressure produced in the soil at the depth of the tube due to the $100 \mathrm{G}$ loading) was designed and fabricated. The intent was to statically verify that the soil container and instrumentation would operate at pressures anticipated from $100 \mathrm{G}$ loading on the centrifuge.

4) The explosive was designed and fabricated based on specifications determined from computer simulations. The explosive charge consisted of PBX9407 pellets glued together to form a column four inches long and attached to a plastic cased EBW detonator (total weight about 2.8 gram).

5) The charge design proof test was performed inside a sample aluminum tube.

6) The soil was prepared and the moisture adjusted to contain about $6 \%$ water.

7) In each experiment, the soil was placed into the container using about 1 inch lifts. Each lift was hand-rolled between layers. When the container was about half full, the aluminum tube (with strain gages and the pressure gages already mounted) was placed horizontally on the soil. Soil was placed and rolled to the tube midline. Accelerometers and instrumentation leads were placed in the soil. The soil addition continued (in lifts) until the container was full.

8) For Test 2, the soil container was stressed using the load frame to that pressure expected from a $100 \mathrm{G}$ load and the 3 gram charge was fired.

9) For Test 3 (a 3 gram charge at $100 \mathrm{G}$ ), the soil container was mounted on the centrifuge. A $100 \mathrm{G}$ run of the centrifuge was conducted with the 3 gram charge loaded in the tube. The 3 gram charge was examined for possible structural damage to the explosive holder or explosive pellets. No damage was seen. The $100 \mathrm{G}$ run was repeated and the 3 gram charge was fired. 


\subsection{Description Of The Large Scale Centrifuge Facility}

Sandia National Laboratories Large Centrifuge Facility (LCF), Figure 2.1, is a state-of-the-art facility in Albuquerque. This facility was developed to simulate loads on weapons components and systems that are produced by missiles and jet aircraft acceleration. Its capabilities also include adaptation for geotechnical experiments. The 29-foot-radius indoor centrifuge has a dynamic load capacity of 1.6 million G-pounds. It can accelerate 16000 pounds to nearly 100 G's, and lighter loads may be accelerated to nearly $300 \mathrm{G}$ 's. The centrifuge maximum rotational velocity is $175 \mathrm{rpm}$ due to aerodynamic limits. The indoor centrifuge room has a 12-foot clearance height.

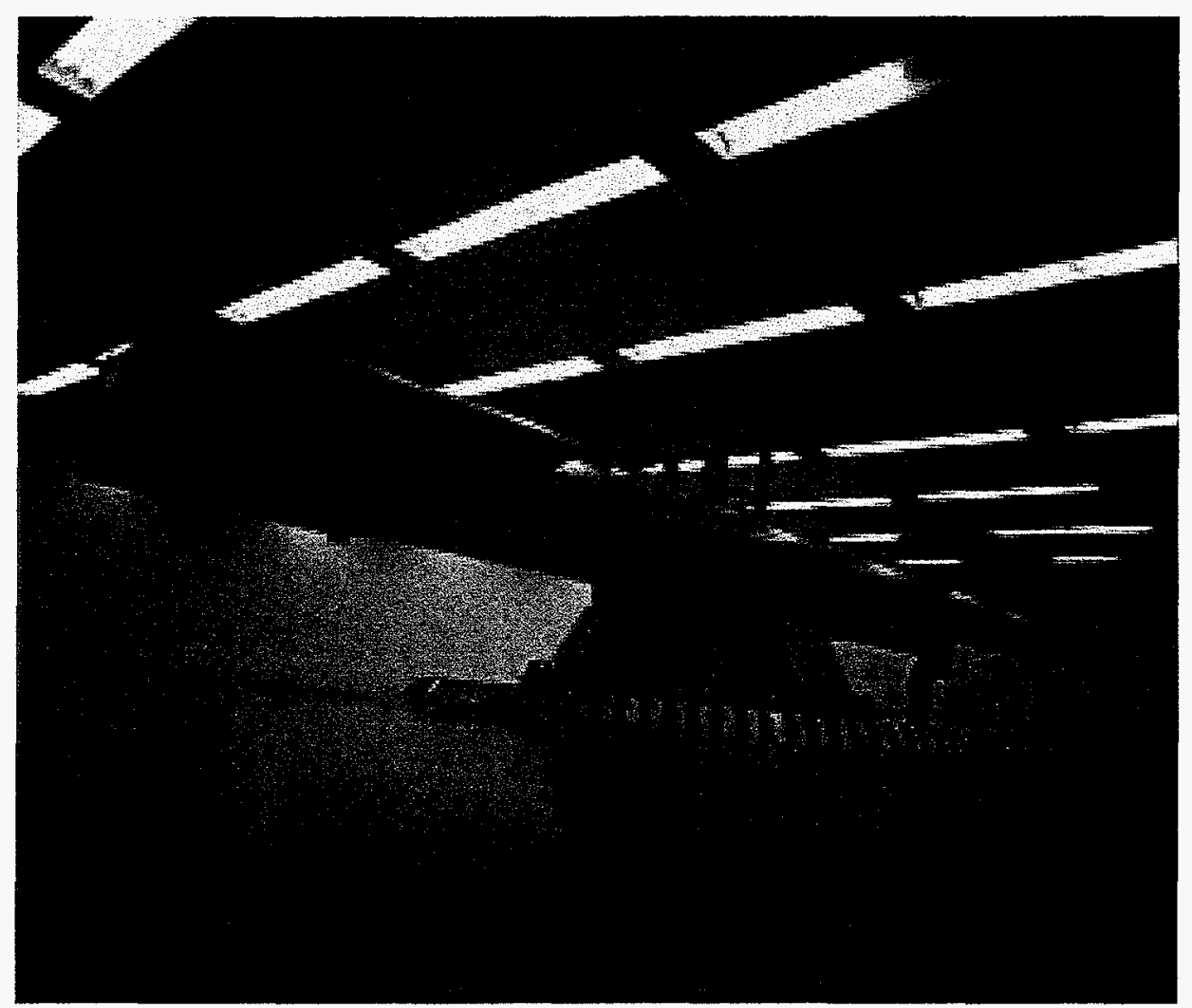

Figure 2.1. Sandia National Laboratories Large Centrifuge Facility.

The Geotechnical Swing-Arm Assembly, Figure 2.2, consists of a pair of swing-arms mounted to pivots that are attached to the centrifuge-arm load-attachment points. The swing-arms allow the geotechnic material to be inserted into a fixture in the normal earth gravitational environment, and then to swing outward due to the centripetal acceleration of the centrifuge. This design allows the resultant acceleration vector to remain in the same orientation as the experiment. The lower end of the swing-arms carry a rigid frame that can accommodate a variety of geotechnical experiment fixtures. The soil fixture used in this experiment is shown securely attached to the rigid frame in Figure 2.2. 


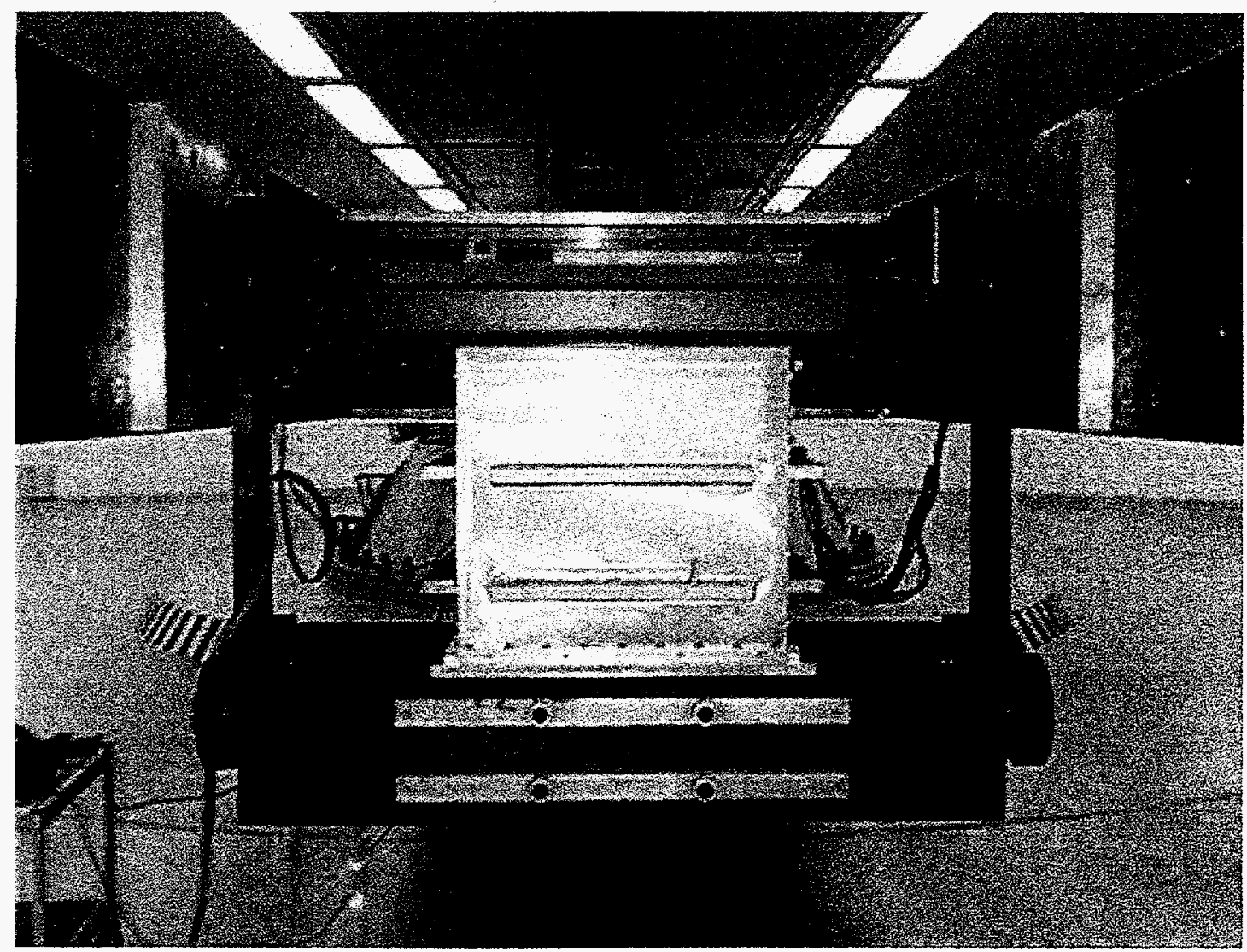

Figure 2.2. The Geotechnical Swing-Arm Assembly.

\subsection{Centrifuge Scaling Relations}

The relationship between properties in a scale model and those in a full-scale prototype is defined by a set of scaling laws. These laws are derived from dimensional analysis of the hydrodynamic equations that govern the phenomena of interest. Consistent sets of scaling laws have been derived for many such hydrodynamic problems (Gaffney 1983, Schmidt and Holsapple 1980). Table 2.1 gives the scaling factors that have been established for basic soil parameters in centrifuge tests.

Table 2.1. Centrifuge scale factors

\begin{tabular}{|c|c|}
\hline Parameter & Scale factor \\
\hline length & $1 / \mathrm{G}$ \\
\hline displacement & $1 / \mathrm{G}$ \\
\hline stress and strain & 1 \\
\hline acceleration & $\mathrm{G}$ \\
\hline time & $1 / \mathrm{G}$ \\
\hline energy & $1 / \mathrm{G}^{3}$ \\
\hline frequency & $\mathrm{G}$ \\
\hline
\end{tabular}


Similarity conditions are expressed in terms of G-values, assuming that acceleration is scaled at $\mathrm{G}$ and the model lengths are scaled at $1 / \mathrm{G}$. Note that Table 2.1 assumes that prototypic material is used in the model, i.e., particle size and density have a scale factor. Note that explosive yields will scale as $1 / \mathrm{G}^{3}$ because the specific energy is constant and the total energy is a product of density and length cubed.

In summary, all dimensions of a model needed to represent a full-scale structure scale inversely proportional to the applied $G$ field, and other quantities scale as shown in Table 2.1. For example, at $100 \mathrm{G}$ 's, one pound of force on a $1 / 100^{\text {th }}$ scale model is equivalent to 10000 pounds on a full-sized object. One gram of explosives at $100 \mathrm{G}$ 's generates an energy level that is equivalent to a million grams, or one metric ton of explosives. 
This page intentionally left blank. 


\subsection{EXPERIMENT DESIGN USING COMPUTER SIMULATIONS}

\section{1 $\quad$ ALEGRA}

The computer code ALEGRA was used in this study to design the size and thickness of the aluminum tunnel liner and the decoupled explosive charge inside the tunnel. ALEGRA is an arbitrary Lagrangian-Eulerian (ALE) wave code with specific emphasis on large distortion and shock propagation (Budge et al, 1997a, Budge et al, 1997b).

\subsection{Preliminary Calculations}

Preliminary calculations were performed using a pseudo one-dimensional ALEGRA model. This model treats the explosive, air, tunnel liner and soil as depicted in Figure 3.1. Computational cells or elements in this model are actually two-dimensional axisymmetric but the boundary conditions on the model constrain it to one-dimensional behavior such as observed when a shock wave is transmitted lengthwise through a metal rod. In this model the explosive is shown as magenta, the air blue, the aluminum tunnel liner as green and the soil as yellow. A gravitational acceleration of $100 \mathrm{G}$ in the negative $\mathrm{X}$ direction was applied to this model.

A Jones-Wilkins-Lee (JWL) equation-of-state, with a programmed burn, was employed to model the PBX-9407 explosive detonation (Budge et al, 1997a). Parameters necessary to characterize the explosive behavior during detonation are given in Table 3.1.

A soil/compressible-foam constitutive model was used to model the engineered soil compacted around the aluminum pipe. Table 3.2 contains the single-value parameters associated with the material model and Table 3.3 shows the pressure versus volumetric strain relationship necessary for the model.

Table 3.1. JWL equation-of-state parameters for PBX-9407

\begin{tabular}{|c|c|c|}
\hline Parameter & Value & Units \\
\hline reference density & 101.12 & $\mathrm{lb} / \mathrm{ft}^{3}$ \\
\hline $\mathrm{a}$ & $8.31 \mathrm{e} 7$ & $\mathrm{psi}$ \\
\hline $\mathrm{b}$ & $2.08 \mathrm{e} 6$ & $\mathrm{psi}$ \\
\hline $\mathrm{c}$ & $1.74 \mathrm{e} 5$ & $\mathrm{psi}$ \\
\hline omega & 0.32 & \\
\hline $\mathrm{r} 1$ & 4.6 & \\
\hline $\mathrm{r} 2$ & 1.4 & $\mathrm{psi}$ \\
\hline cj pressure & $3.84 \mathrm{e} 6$ & $\mathrm{ft} / \mathrm{s}$ \\
\hline detonation velocity & $2.59 \mathrm{e} 4$ & $\mathrm{~K}$ \\
\hline cj temperature & 4962 & \\
\hline
\end{tabular}


Table 3.2. Soil and crushable foam constitutive model parameters for engineered soil

\begin{tabular}{|c|c|c|}
\hline Parameter & Value & Units \\
\hline initial density & 86.95 & $\mathrm{lb} / \mathrm{ft}^{3}$ \\
\hline bulk modulus & $2.90 \mathrm{e} 3$ & $\mathrm{psi}$ \\
\hline shear modulus & $9.28 \mathrm{e}-3$ & $\mathrm{psi}$ \\
\hline $\mathrm{a} 0$ & $5.51 \mathrm{e} 5$ & \\
\hline $\mathrm{a} 1$ & 0.95 & \\
\hline $\mathrm{a} 2$ & 0.00 & \\
\hline
\end{tabular}

Table 3.3. Pressure versus volumetric strain for engineered soil

\begin{tabular}{|c|c|}
\hline Volumetric Strain & Pressure (psi) \\
\hline $6.0000 \mathrm{e}-3$ & 3.47 \\
\hline 0.0200 & 6.94 \\
\hline 0.0640 & 13.88 \\
\hline 0.1140 & 27.69 \\
\hline 0.2231 & 50.68 \\
\hline 0.2400 & 55.48 \\
\hline 0.2600 & 83.27 \\
\hline 0.2700 & 110.96 \\
\hline 1.0000 & 2154.28 \\
\hline
\end{tabular}

An elastic-perfectly-plastic constitutive model was employed to model the aluminum tube that served as the tunnel liner after the emplacement of the soil around the tube. The constitutive model parameters selected for aluminum are given in Table 3.4.

Table 3.4. Elastic-perfectly-plastic constitutive parameters for aluminum tube

\begin{tabular}{|c|c|c|}
\hline Parameter & Value & Units \\
\hline Youngs modulus & $9.99 \mathrm{e} 6$ & psi \\
\hline Poissons ratio & 0.334 & psi \\
\hline yield stress & $4.00 \mathrm{e} 4$ & psi \\
\hline hardening modulus & $1.48 \mathrm{e} 5$ & \\
\hline beta & 1.0 & \\
\hline
\end{tabular}

The final material behavior model necessary for this simulation was for the air that decoupled the explosive from the tunnel liner. This was treated as an ideal gas with parameters given in Table 3.5.

Table 3.5. Ideal gas parameters for air

\begin{tabular}{|c|c|c|}
\hline Parameter & Value & Units \\
\hline reference density & 0.08 & $\mathrm{lb} / \mathrm{ft}^{3}$ \\
\hline gamma & 1.4 & $\mathrm{~K}$ \\
\hline reference temperature & 288.2 & \\
\hline $\mathrm{cv}$ & $0.7178 \mathrm{e} 4$ & \\
\hline
\end{tabular}


Variables that could be adjusted in this model included: 1) explosive radius, 2) tunnel radius, and 3) tunnel liner thickness. A tunnel diameter of 1.5 inches was chosen based on the general physical dimensions of the existing centrifuge swing-arm which constrained the size of the container holding the tunnel experiment. The container was sized to be a cube two feet on each side. The two foot depth with the tunnel in the center dictated one foot of soil above and below the tunnel. Experience indicated that the tunnel diameter should be approximately $1 / 10$ the length of the soil surrounding it to control the influence of the upper (free) surface and lower (fixed) surface. The tunnel diameter was thus set at 1.5 inches.

A steady-state acceleration environment of $100 \mathrm{G}$ on the centrifuge produces the same stresses and deformations in the soil and tunnel as would be observed in a model with all lengths multiplied by 100 . This model thus simulates a 150 inch (12.5 feet, $3.8 \mathrm{~m}$ ) diameter tunnel buried 100 feet $(30.5 \mathrm{~m})$ deep.

Experience indicated that the diameter of the explosive would be an important explosive parameter influencing the effect on the tunnel liner. This was proven later by both the twodimensional calculations and the experiment itself. Thus, with tunnel diameter and depth-ofburial already determined, the two variables that could be adjusted in the experiment were explosive diameter and tunnel liner thickness.

It was decided that the criteria for controlling the combination of explosive diameter and tunnel liner thickness should be measurable plastic deformation of the tunnel liner resulting from explosive detonation inside the tunnel. The tunnel liner thickness and explosive diameter were both constrained by the commercially available sizes. A number of simulations were performed with several combinations of explosive diameter and tunnel thickness using ALEGRA and the pseudo one-dimensional model. The best combination arrived at was a tunnel liner thickness of 0.083 inches $(0.2108 \mathrm{~cm})$ and an explosive diameter of 0.1875 inches of PBX-9407. The length of the explosive was set at 3.75 inches $(9.525 \mathrm{~cm}$ ) which resulted in a total explosive weight of $2.75 \mathrm{~g}$. At a steady-state acceleration environment of $100 \mathrm{G}$ on the centrifuge, this small explosive is equivalent to about $6000 \mathrm{lb}$ of TNT.

\subsection{Detailed Two-Dimensional Simulations}

All of the design parameters discussed above were employed in a more detailed two-dimensional ( $\mathrm{x}$ - tunnel diameter, $\mathrm{y}$ - tunnel length) axisymmetric model of the tunnel experiment on the centrifuge. A close-up of the two-dimensional axisymmetric ALE model is shown in Figure 3.2. In this model the explosive is displayed in magenta, air blue, aluminum green and soil yellow, the same as the one-dimensional model of Figure 3.1. The material models for the explosive, air, aluminum and soil used in the two-dimensional model are exactly the same as those given above for the pseudo one-dimensional model. Detonation of the explosive is designated to occur along the line on the bottom of the explosive. This model also has a gravitational acceleration of $100 \mathrm{G}$ in the negative $\mathrm{X}$ direction. 
Pressure caused by the detonation of the explosive and the deformation of the aluminum liner from times 0.0 to 245 micro-seconds $(\mu \mathrm{s})$ is shown in Figures 3.3 through 3.12. In these plots the pressure corresponding to red has been set at $1.0 \mathrm{E} 9$ dynes $/ \mathrm{cm}^{2}$ (14500 psi) Pressures greater than this value are also plotted as red. The actual maximum pressure and its location are shown with symbols on the plot and the value corresponding to the symbol is given below the color bar. A number of very interesting phenomenon can be observed in this series of figures. In Figure 3.4 ( $25 \mu \mathrm{s})$ the air is being pushed ahead of the explosive gas because of the large density difference between the two. In Figure $3.5(29 \mu \mathrm{s})$ the air has been compressed by the explosive gas against the aluminum liner and begins to rebound as illustrated in Figures 3.6-3.7. In Figure $3.8(39 \mu \mathrm{s})$ the air is reconverging in the center of the tube where the explosive was before detonation. Thus, the shock wave marking the separation between the air and explosive gas reverberates a number of times in the tube. The majority of the energy imparted to the tube resulting in plastic deformation is expended during the initial impact of the explosively induced shock wave on the aluminum tube. Plastic deformation of the tube can be seen starting as early as $29 \mu \mathrm{s}$. Transmission of the explosively induced shock wave through the tunnel liner and into the soil is observed in Figure 3.11 and especially in Figure 3.12 where the plotting pressure range has been significantly reduced to highlight lower pressures. The final predicted deformed shape of the tube is shown in Figure 3.13. The tube flare is the same length as the explosive and the outward displacement is constant indicating that the pseudo one-dimensional ALEGRA model used for the design of this experiment was an acceptable approach. The final outward radial displacement predicted by the one-dimensional model was $0.24 \mathrm{~cm}$ compared with $0.14 \mathrm{~cm}$ calculated from the two-dimensional model. The explosive design proof test was performed with the explosive charge detonated inside an aluminum tube that was wrapped with rubber (Appendix D). The outward radial displacement of the tube in the proof test was $0.15 \mathrm{~cm}$. 


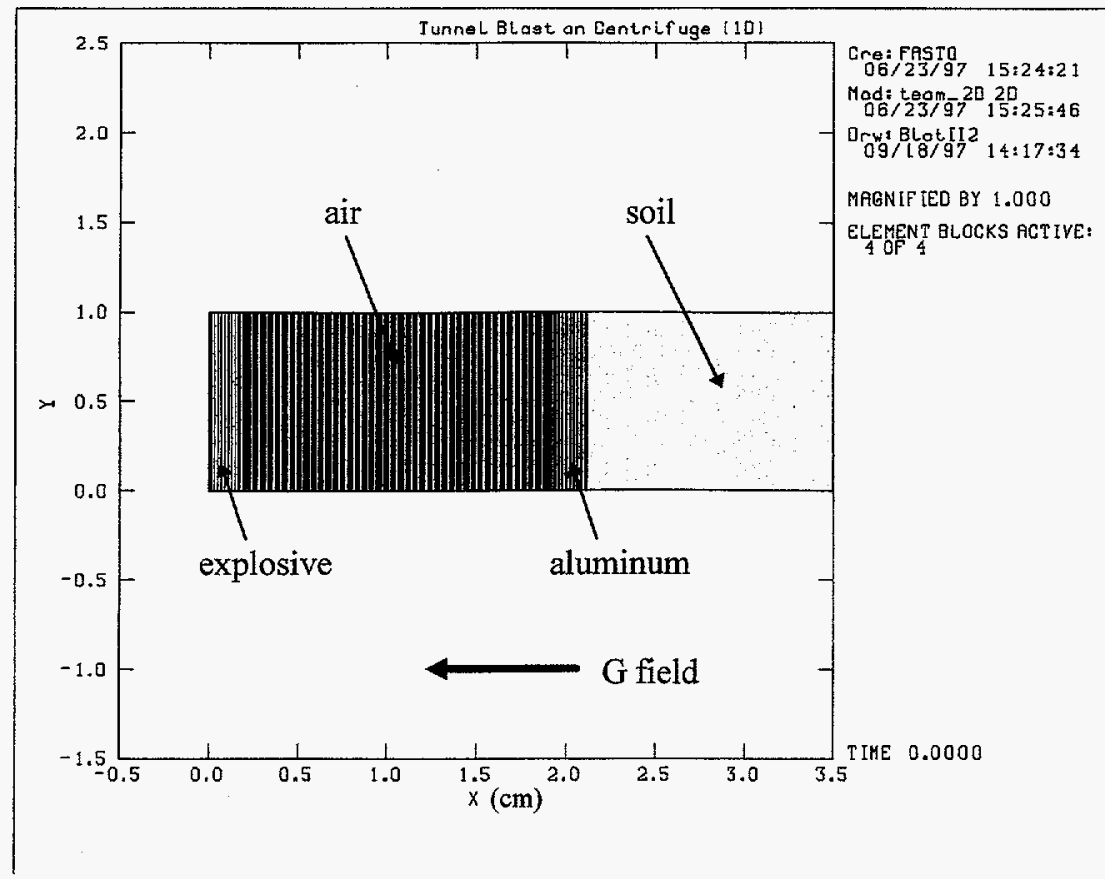

Figure 3.1. Pseudo one-dimensional ALE model used to design the decoupled explosive tunnel experiment. The explosive is represented as magenta; air, blue; aluminum, green; soil, yellow.

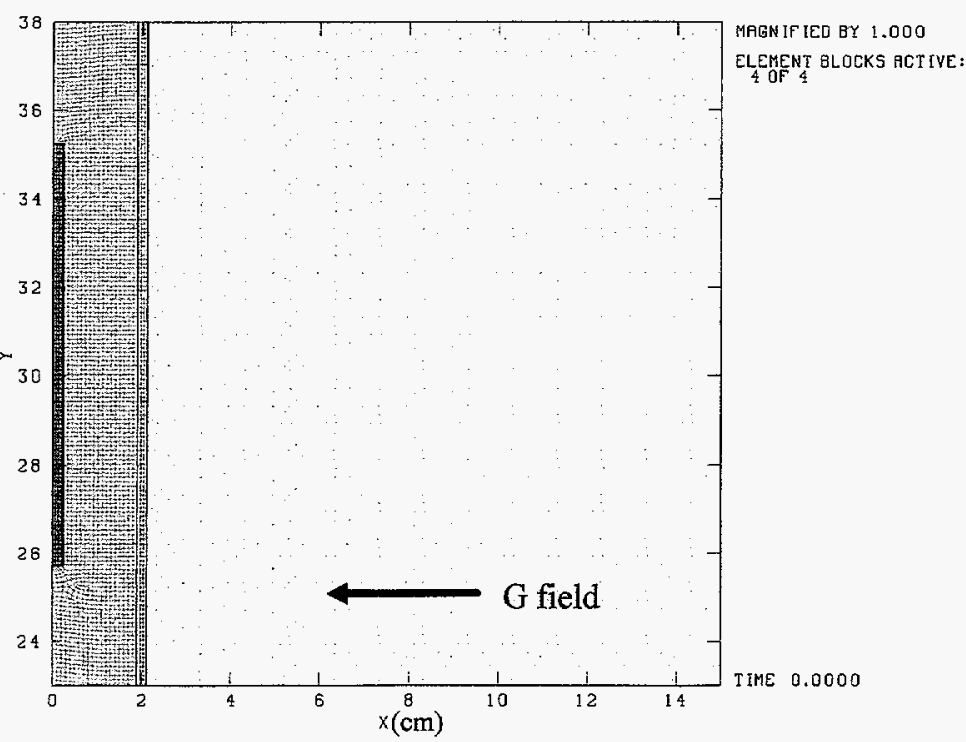

Figure 3.2. Two-dimensional ALE model used to predict the response of the model to a decoupled explosive in a tunnel. 


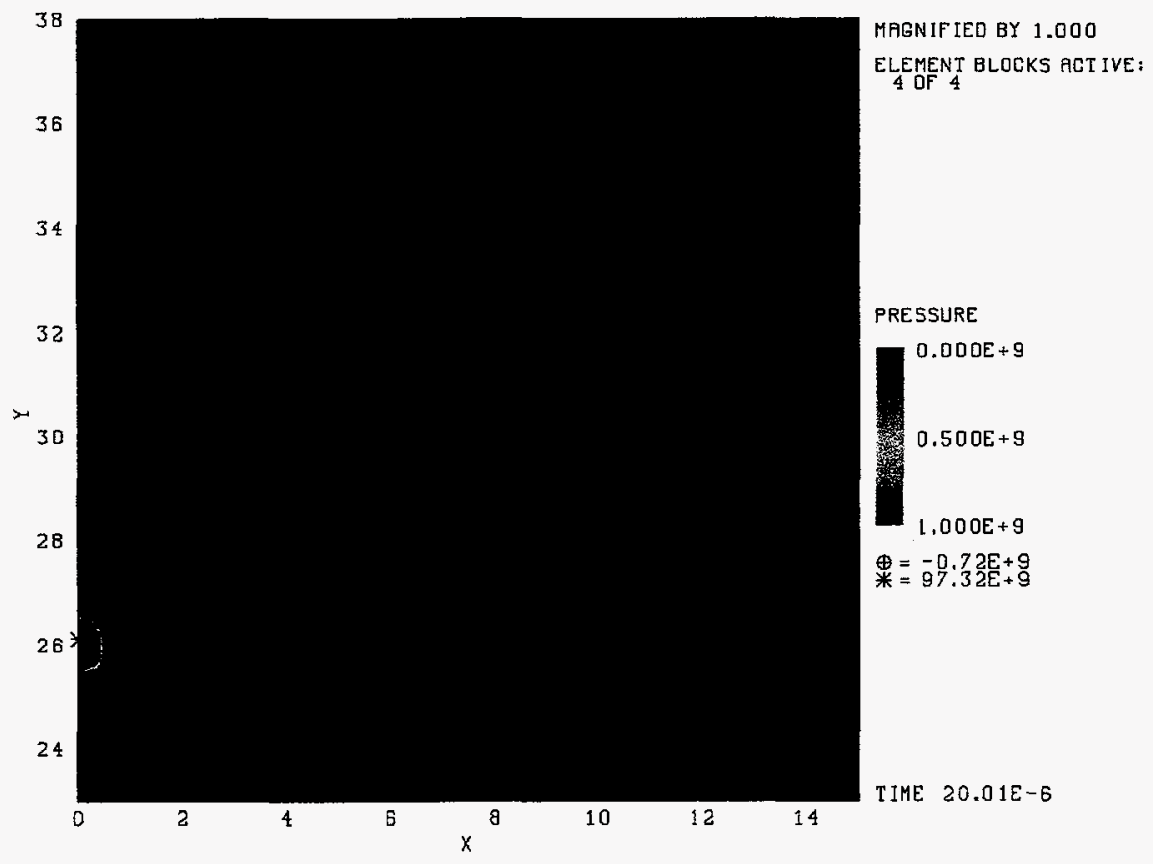

Figure 3.3. Pressure induced by the explosive at $20 \mu \mathrm{s}$.

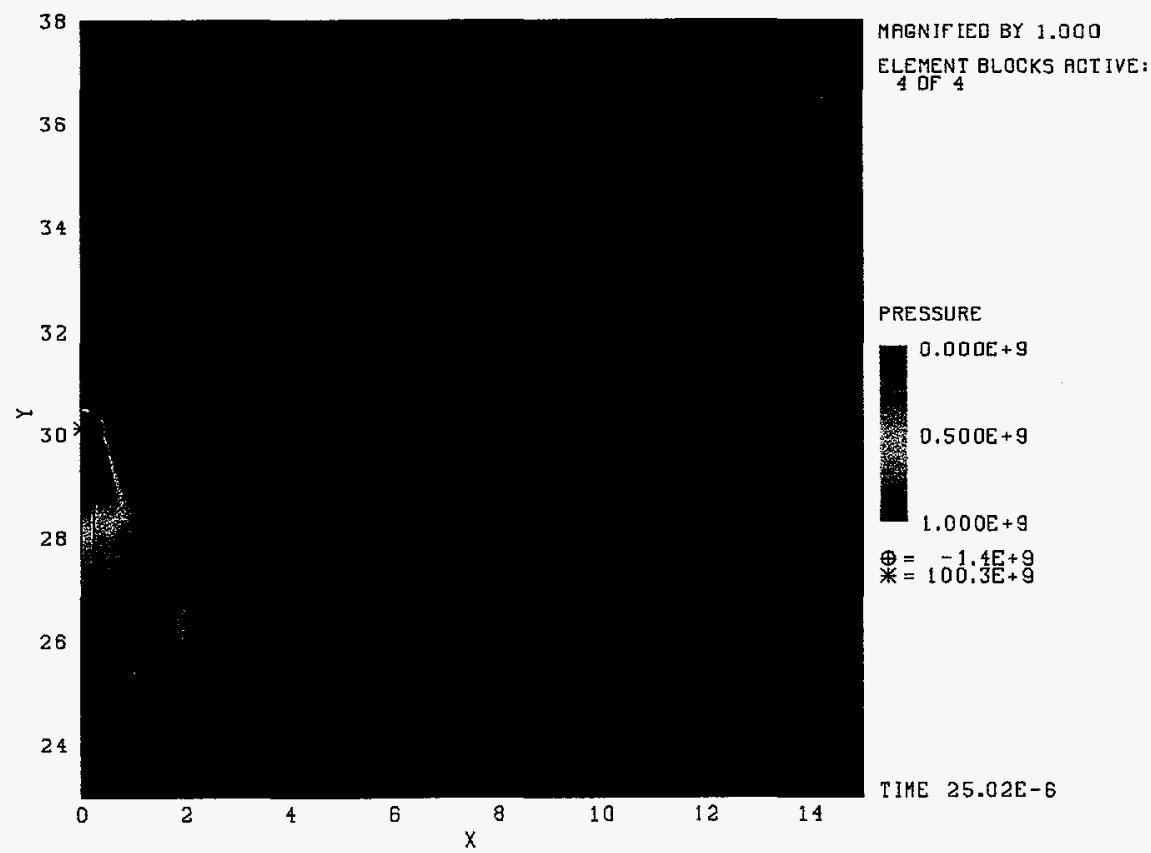

Figure 3.4. Pressure induced by the explosive at $25 \mu$ s. 


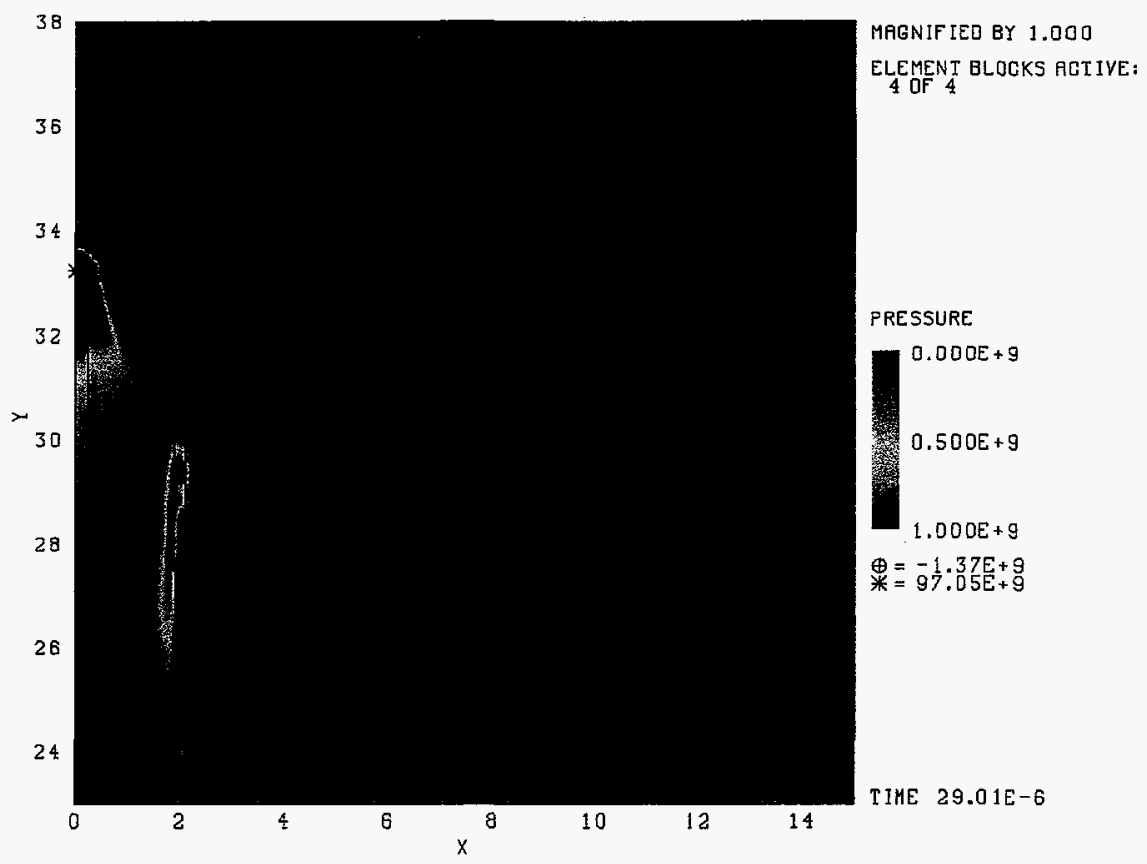

Figure 3.5. Pressure induced by the explosive at $29 \mu$ s.

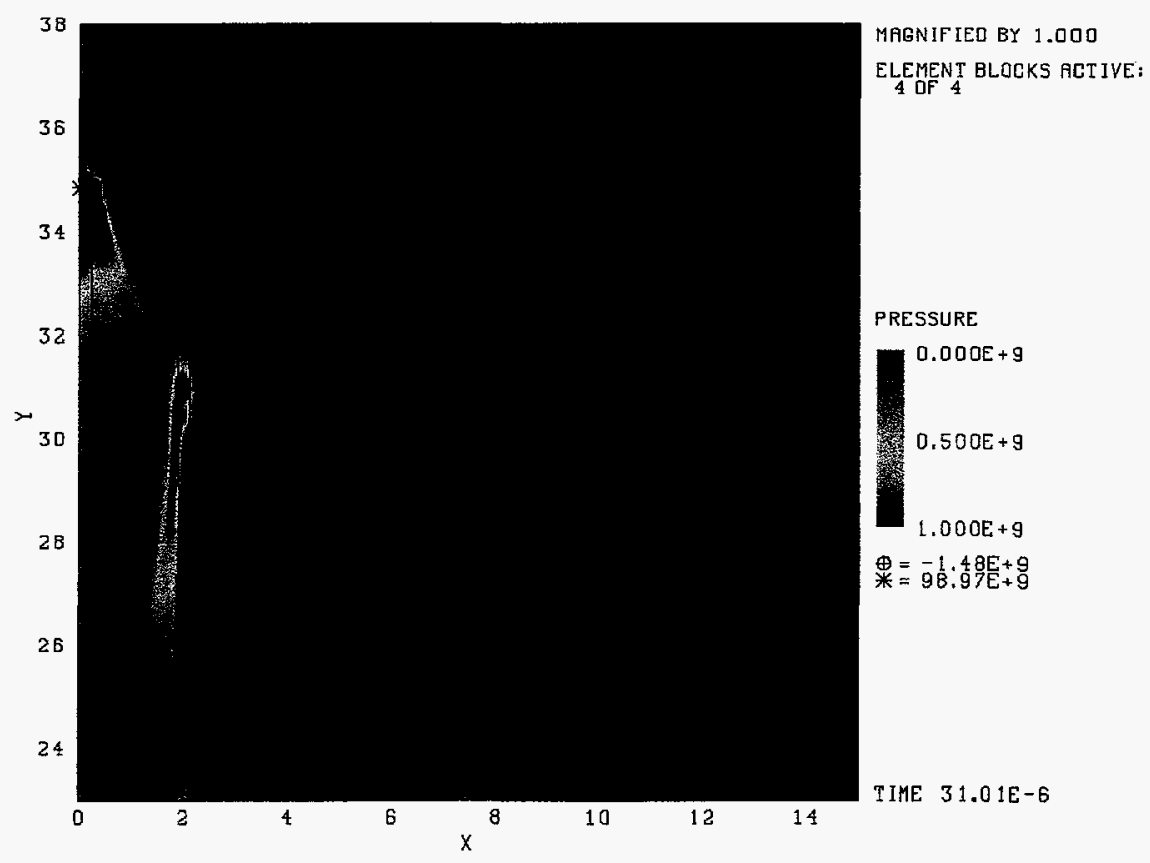

Figure 3.6. Pressure induced by the explosive at $31 \mu \mathrm{s}$. 


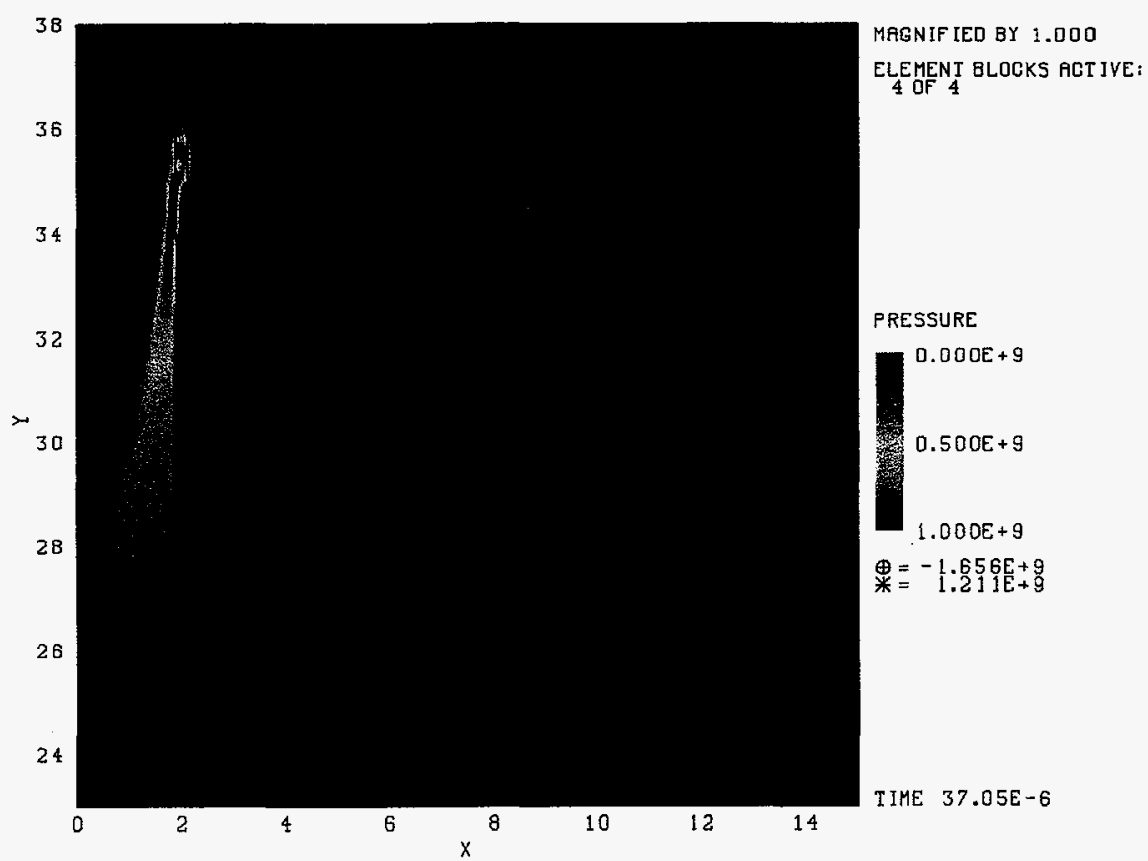

Figure 3.7. Pressure induced by the explosive at $37 \mu$ s.

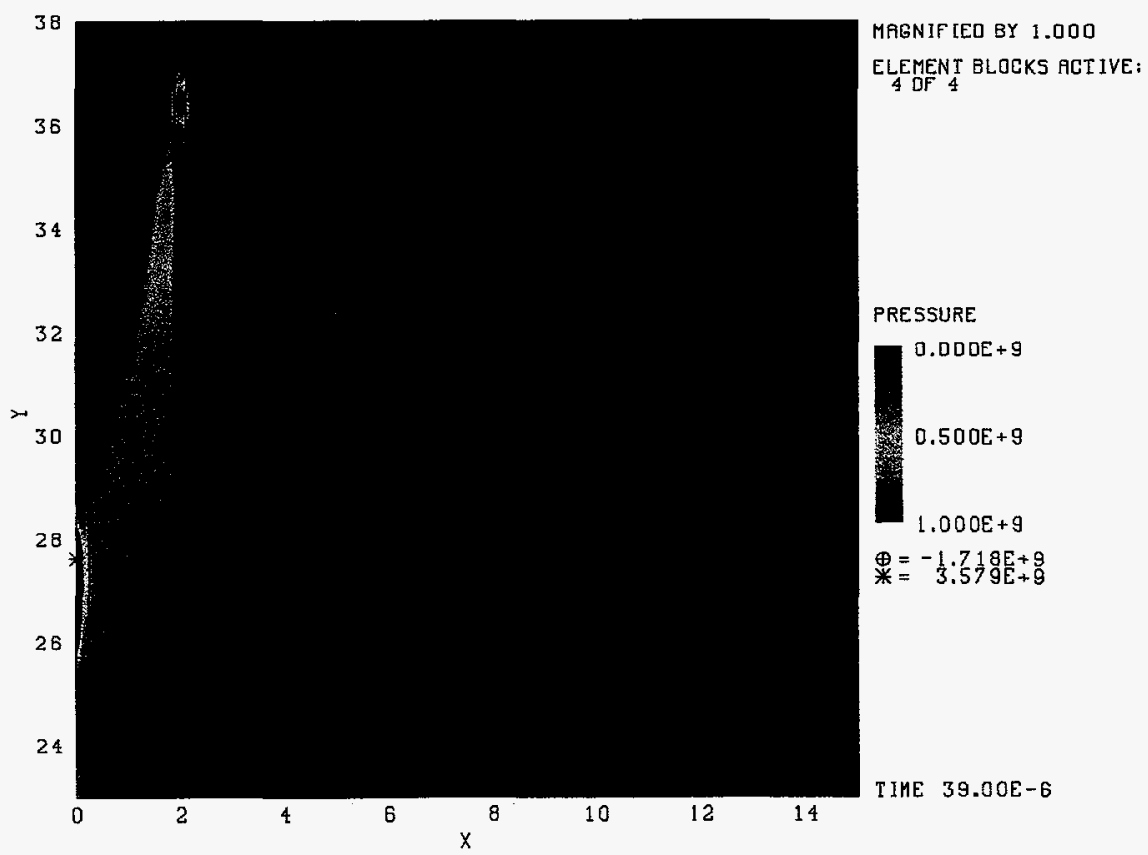

Figure 3.8. Pressure induced by the explosive at $39 \mu$ s. 


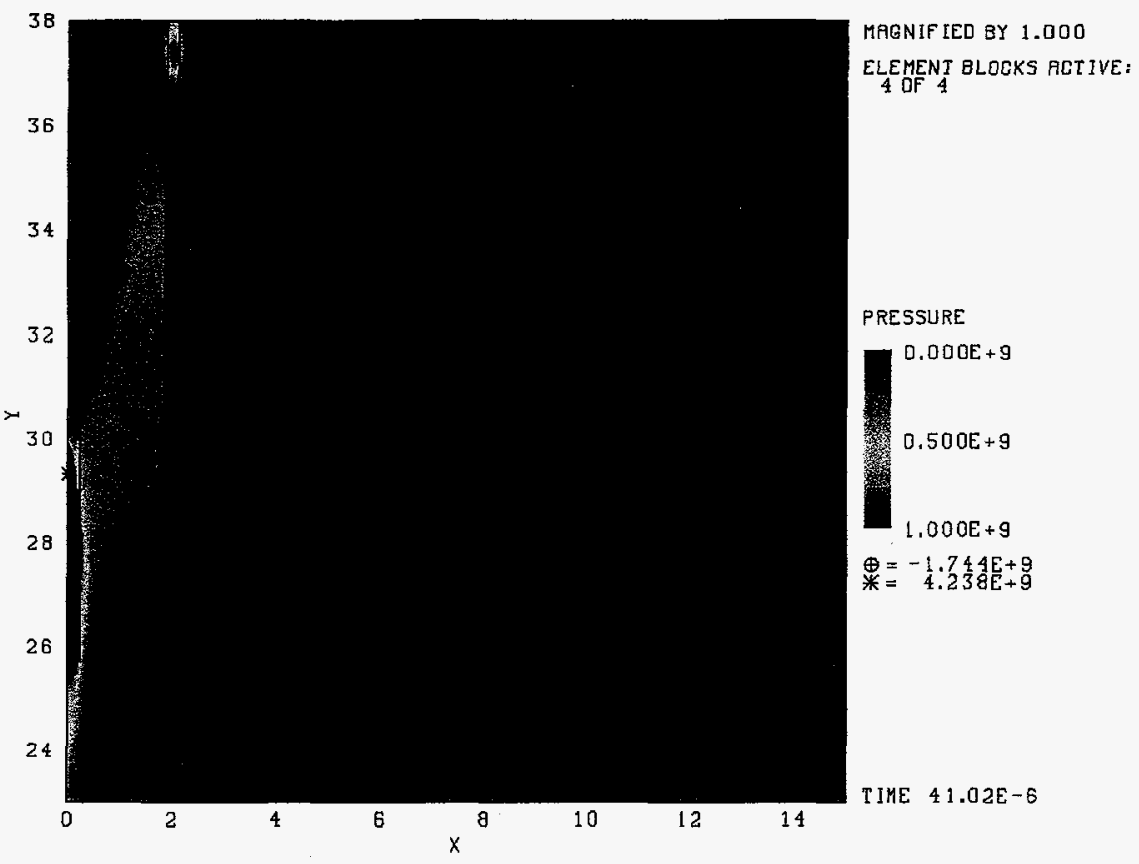

Figure 3.9. Pressure induced by the explosive at $41 \mu \mathrm{s}$.

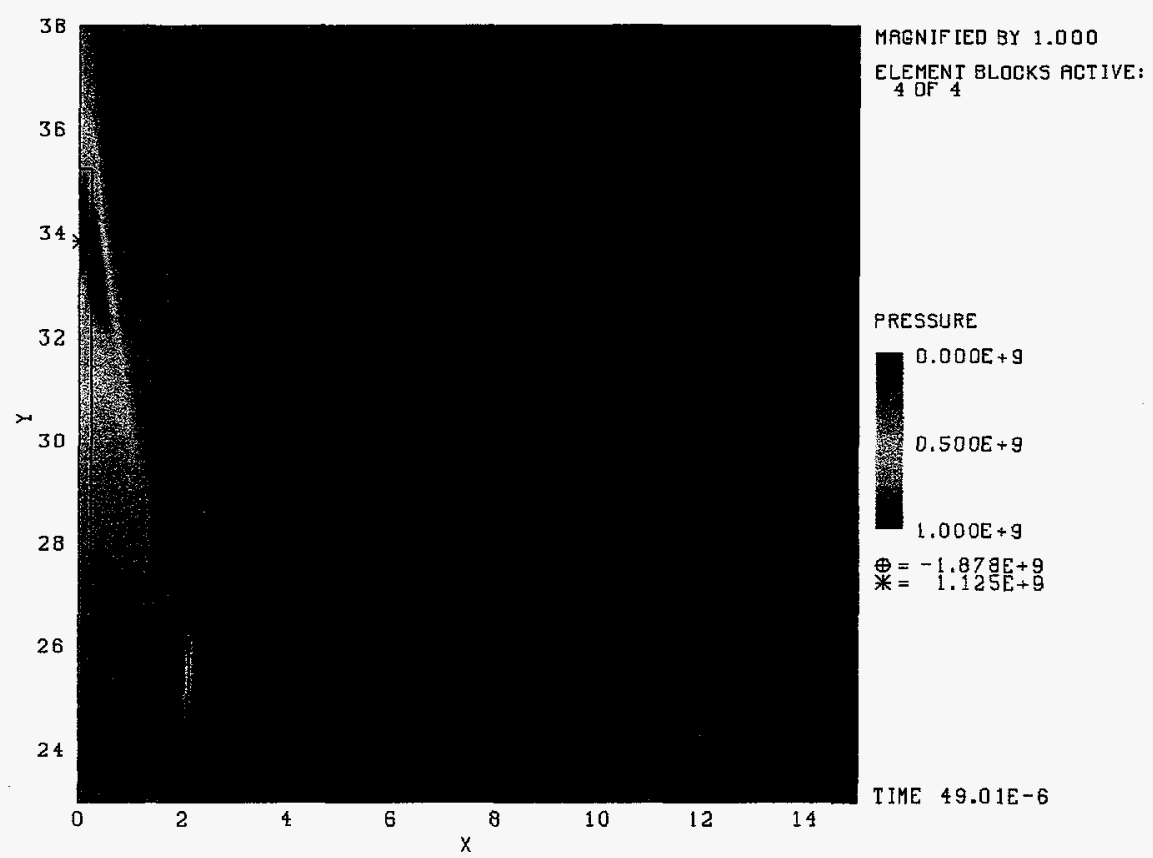

Figure 3.10. Pressure induced by the explosive at $49 \mu \mathrm{s}$. 


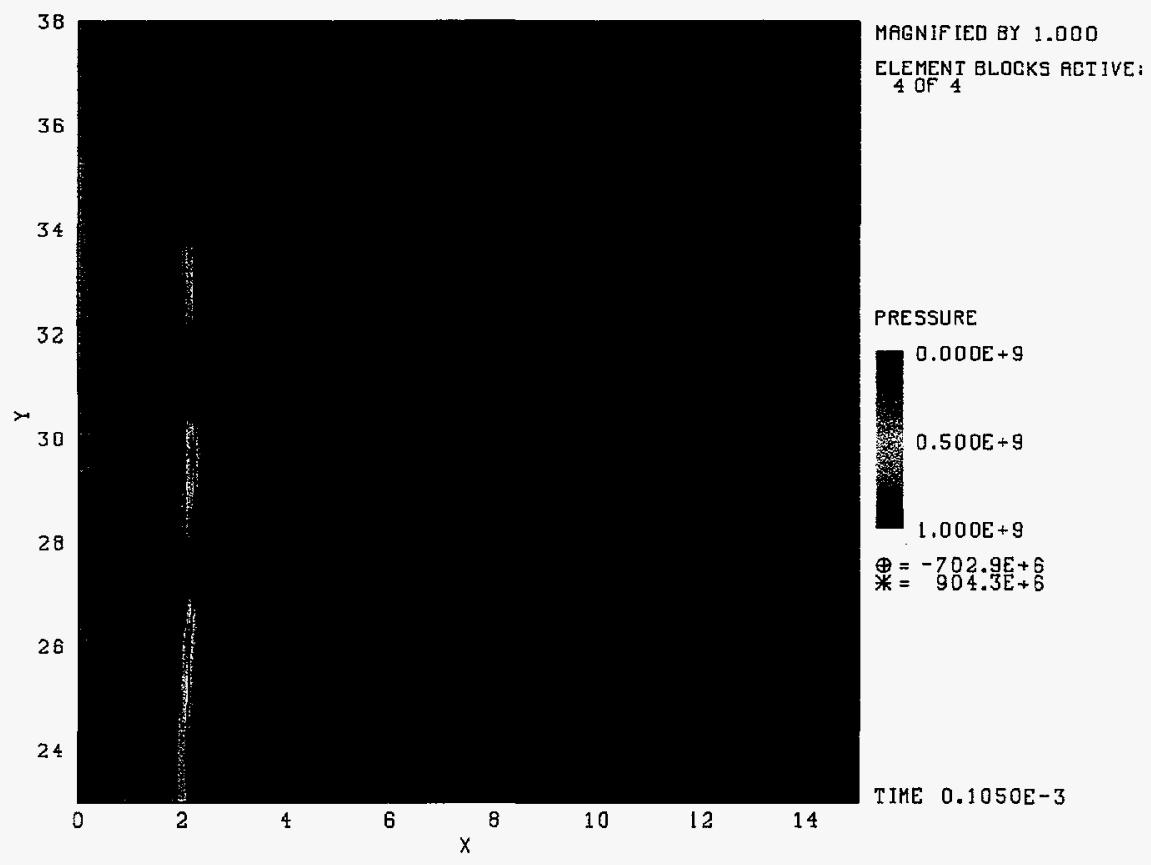

Figure 3.11. Pressure induced by the explosive at $105 \mu$ s.

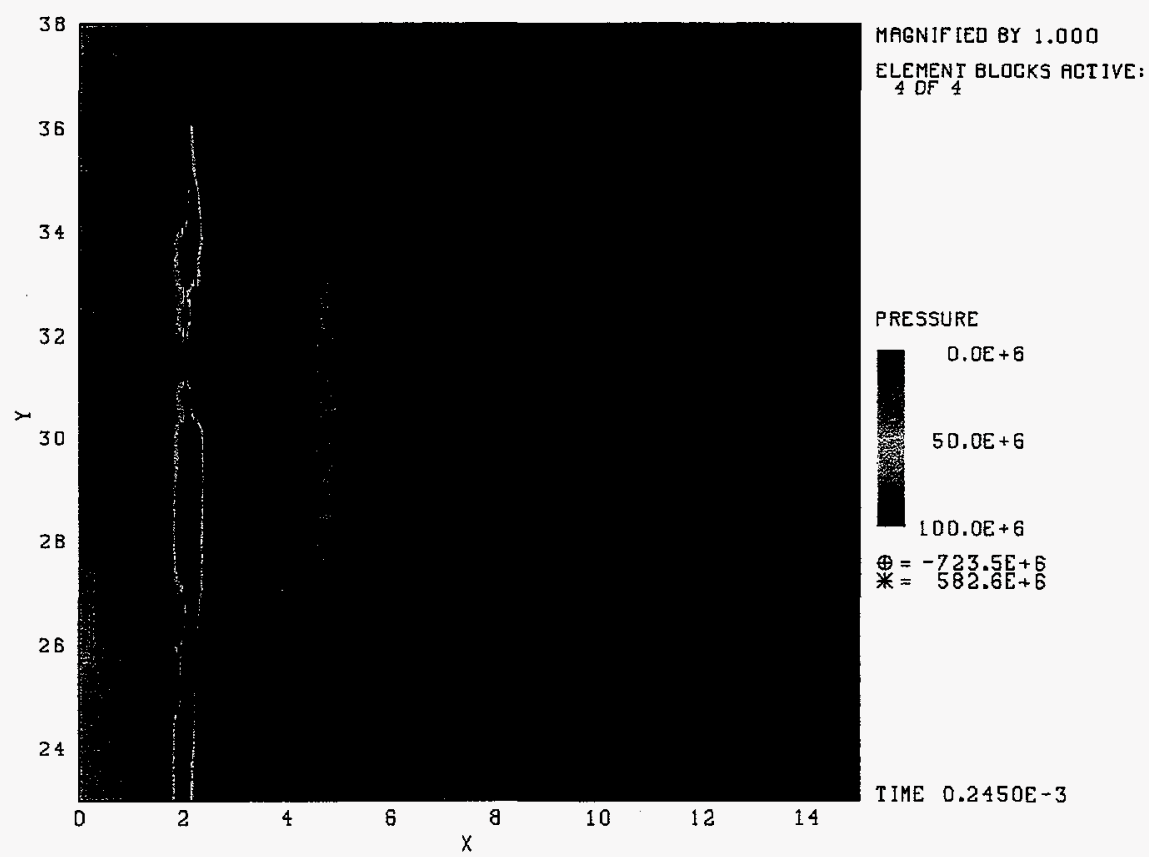

Figure 3.12. Pressure induced by the explosive at $245 \mu \mathrm{s}$. Note the difference in the pressure range to highlight the shock wave moving outward through the soil. 


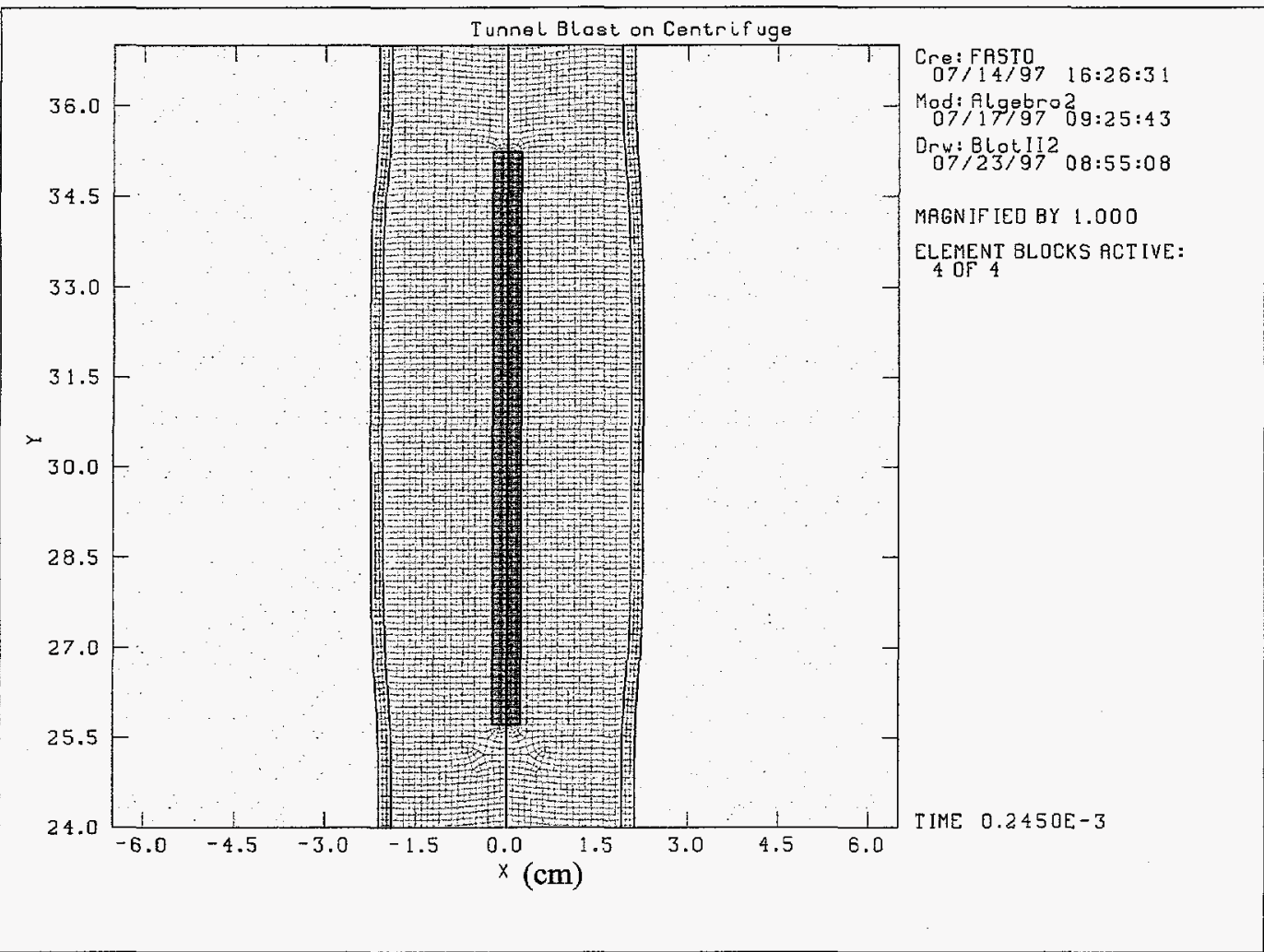

Figure 3.13. Final deformed shape predicted for the aluminum tube. 
This page intentionally left blank. 


\subsection{EXPERIMENTAL ACTIVITIES}

\subsection{Introduction}

The following sections describe the experimental activities which culminated in a fully instrumented model validation experiment conducted at $100 \mathrm{G}$ 's with a 3 gram charge. Initial efforts were dedicated to adjustments in the instrumentation system, which were necessary to minimize electrical noise induced into the measured data by the fireset pulse. A variety of nonexplosive fireset loads were used to exercise the instrumentation system, resulting in several changes being made to the initial configuration. In parallel with the instrumentation development activities, a partially instrumented soil fixture was assembled. This included an aluminum tube with two strain gages and a pressure transducer, and three accelerometers in the soil. These were assembled into the soil container as described in Appendices A, B, and C. Once the final instrumentation configuration was determined, a live detonator was fired inside this test assembly. This test served as a further verification of the adequacy of the instrumentation system, and demonstrated the ability to successfully fire the detonator. In the next test, the same test assembly was used, but a 3 gram charge was detonated, while the test assembly was subjected to a static compressive load which simulated the pressure in the soil that would result in a $100 \mathrm{G}$ acceleration field. This test provided a structural proof test for the container design, and demonstrated the ability to measure strain and acceleration data due to the explosive event. Following this test, the experiment assembly was installed on the centrifuge geotechnic swing fixture. This fixture had been resurrected from an earlier test program. Two centrifuge runs were conducted. On the first run, a detonator was fired to demonstrate proper control of the fireset through the slip rings while the centrifuge was operating. On the second run, a 3 gram charge assembly was placed into the tube, but not fired, to demonstrate the structural integrity of the explosive assembly during a $100 \mathrm{G}$ loading. Finally, the fully instrumented soil fixture was assembled as described in Appendix $\mathrm{C}$. In this experiment, a 3 gram charge was detonated in the instrumented tube, while being subjected to a $100 \mathrm{G}$ acceleration field. The measured data was compared to analytical model results.

\subsection{The Instrumentation System}

Figure 4.1 shows a schematic of the instrumentation system that was used for the experimental measurements. Figure 4.2 shows that the entire system was installed into the instrumentation rack at the center of the centrifuge, which allowed the data to be digitally acquired on-board the centrifuge, instead of passing an analog signal through the slip rings. The digital acquisition was accomplished with two Tektronix TDS744 digitizing oscilloscopes which were set at 100 Msamples/sec, with a $20 \mathrm{MHz}$ bandwidth setting. Each data record was $50 \mathrm{~K}$ points per channel. Pacific 8656 amplifiers were used to provide excitation voltage (10 volts) and amplification (gain of 10) for the accelerometers and strain gages. A pulsed power supply was used to condition the signal from the carbon pressure gage. As shown in Figure 4.1, the scopes and pulsed power supply were triggered by the attenuated fireset pulse. This trigger pulse was also conditioned by ferrite core isolator (Balun isolator). 


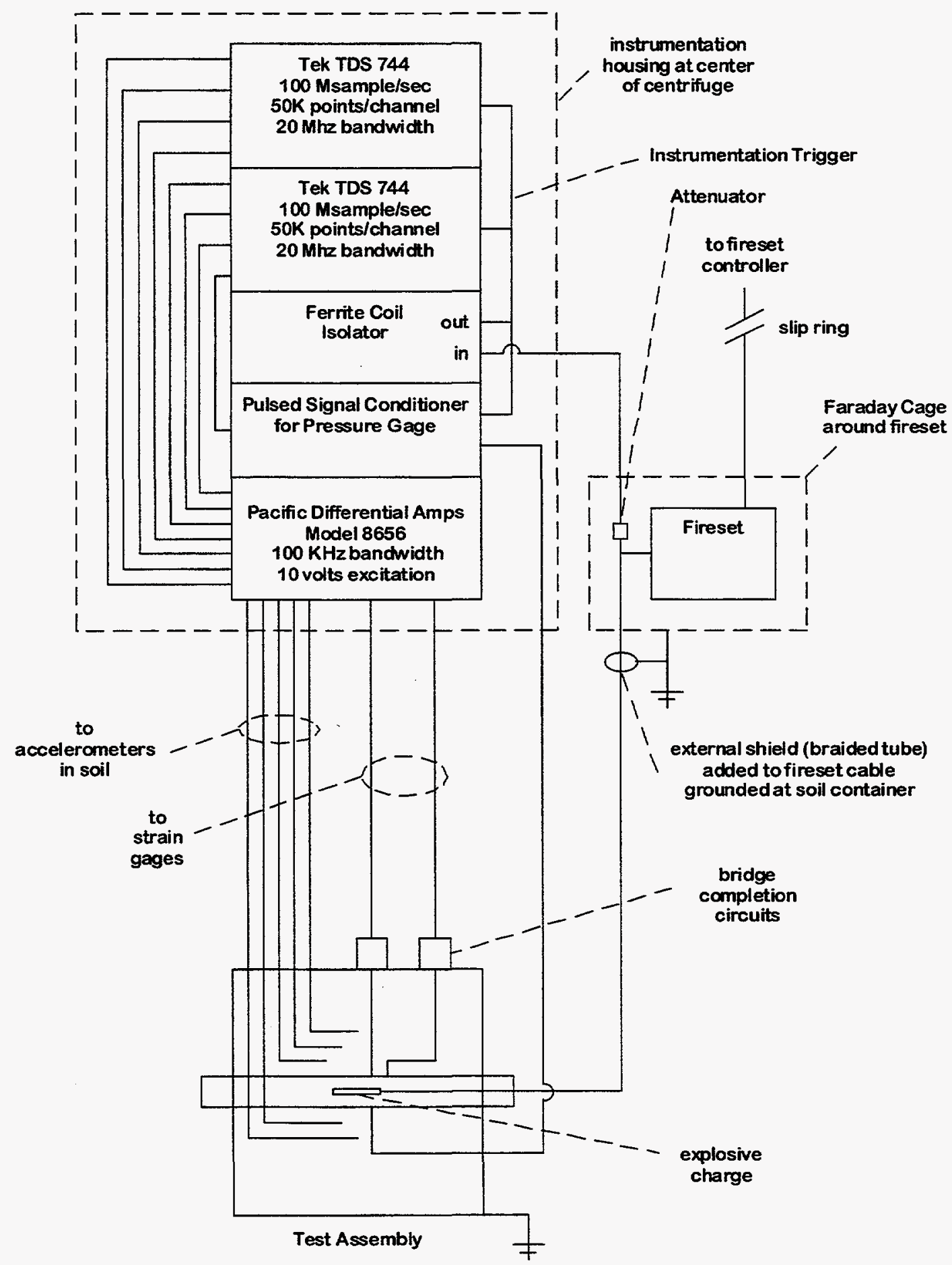

Figure 4.1 Instrumentation schematic. 


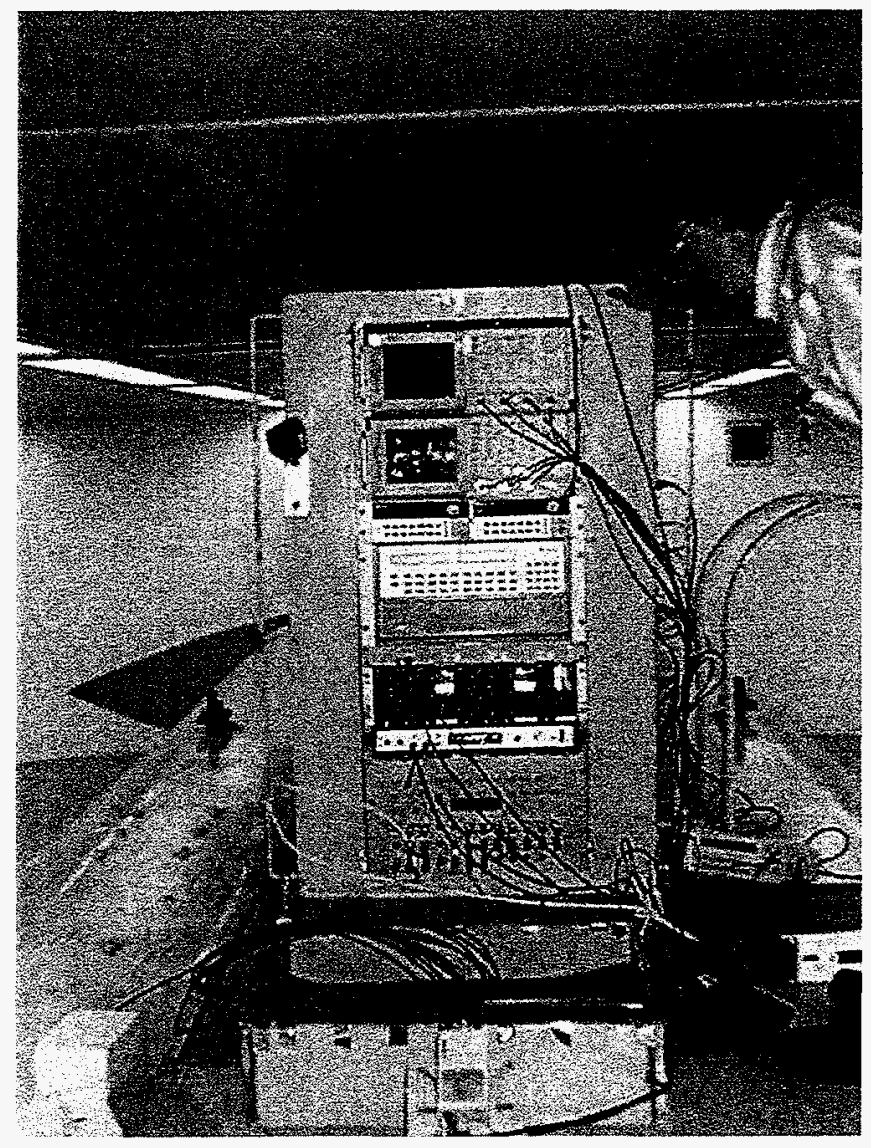

Figure 4.2 The instrumentation rack installed on the center of the centrifuge.

This final instrumentation configuration was determined only after extensive iteration. The initial configuration was evaluated by recording strain and accelerometer data during the firing of a bridgewire. Severe interference was recorded on the data channels due to electrical noise induced by the fireset pulse. The fireset pulse initially produced a large amplitude decayed resonance on the time history. Also, the FS17 fireset "re-striked" after about $200 \mathrm{ms,} \mathrm{causing}$ additional interference at this time. The re-strike problem was corrected by replacing the FS17 with a TC894 fireset. Several changes to the instrumentation system were eventually implemented which improved the initial resonance induced onto the data. The changes were determined over the course of several days in which each change was evaluated. The following is a list of changes implemented that made a noticeable improvement to the interference:

1. The DET cable and fireset were placed in a Faraday cage which was grounded at the soil container. This was accomplished by placing the fireset inside a metal electrical enclosure, and by placing the DET cable inside a tubular braided grounding strap. The fireset case and the shield of the coaxial DET cable were electrically floating (i.e. not grounded).

2. The attenuated fireset pulse which was used to trigger the scopes and pulsed power supply, was passed through a ferrite core coil (Balun). This provided significant improvement by 
increasing common mode noise rejection on the single ended scopes. Note that the signal lows and trigger low were common.

3. The negative excitation wire for each accelerometer and strain gage were electrically connected to the shield of the respective cable, as close to the transducer as possible. Each shield was then grounded at the soil container.

There was an attempt use isolation transformers to provide power to the data acquisition equipment, but these did not yield noticeable improvement.

\subsection{Transducers}

The following types of transducers were used for the indicated measurements:

Soil acceleration

Aluminum tube strain

Aluminum tube OD radial pressure
Endevco $7270 \mathrm{~A}$ piezoresistive accelerometers (200K or $60 \mathrm{~K}$ range)

Micro-Measurements EP-08-250BF-350 high

elongation strain gage

Dynasen PC300.50.EKRTE carbon pressure gage

The strain gages and carbon pressure gage were bonded to the aluminum tube near its midpoint, using Micro-Measurements M-Bond AE-10 epoxy. After the adhesive cured, the strain and pressure gages were covered with four layers of electrical tape to help distribute soil loads and minimize the possibility of damage from soil particles. The accelerometers were buried in the soil at various radial distances from the tube. The following sections contain more details on the location of the accelerometers. Particular attention was given to cable routing between the transducers and exit from the soil container, since there was several inches of movement of the tube as the soil was compressed. Figure 4.3 shows the technique used to accommodate this large movement. The lower part of each cable was taped to the $1 / 8$ inch thick end plate which moved with the tube, and the upper part was taped to the top edge of the soil container. The loops shown were lightly taped in place and covered with a sheet of polyethylene.

\subsection{Test 1 - Static Test with Detonator}

This test served as a final systems check of the instrumentation system. The partially instrumented soil assembly included two strain gages and one pressure gage on the tube, and three accelerometers in the soil. Figure 4.4 shows the gage locations for this test. An RP-2 detonator was placed inside the aluminum tube, approximately 6 inches from the end. Since this assembly was also needed for Test 2 , the detonator was placed away from the middle of the tube to avoid damaging the strain and pressure gages. In addition, the mechanical excitation of the transducers is significantly later in time (relative to the detonator pulse) than it would be for the 3 gram charge configuration. This time delay allowed any induced fireset noise to be separated 
from the actual mechanical data. The initial ringing decayed to an acceptable level in less than $10 \mu \mathrm{s}$, which was the expected time of arrival of the pressure pulse on the inside of the tube. No pressure data was measured. It is suspected that a lead wire had been damaged during assembly.

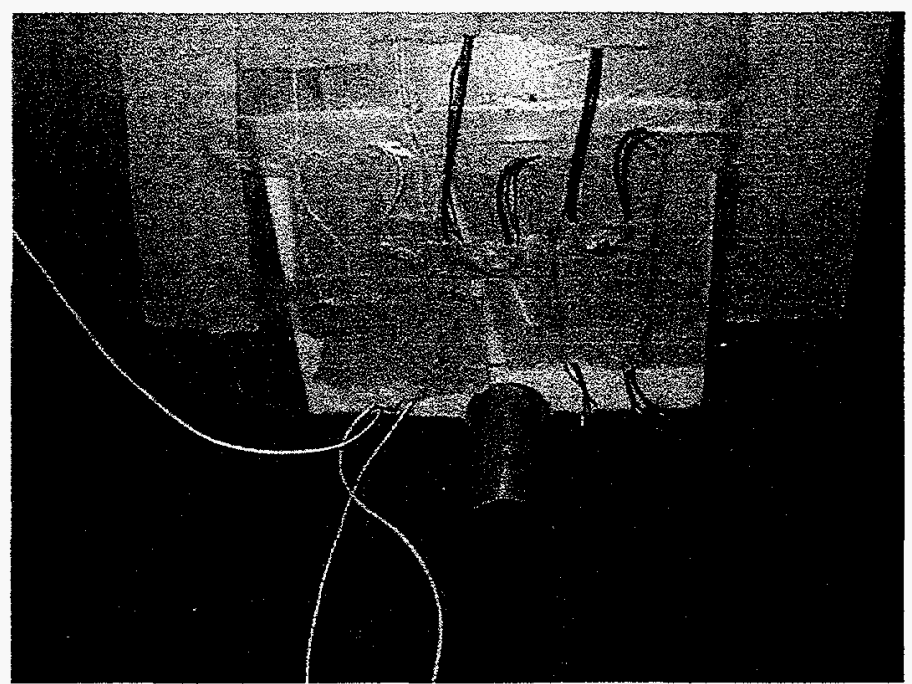

Figure 4.3. The technique used to accommodate large tube movement.

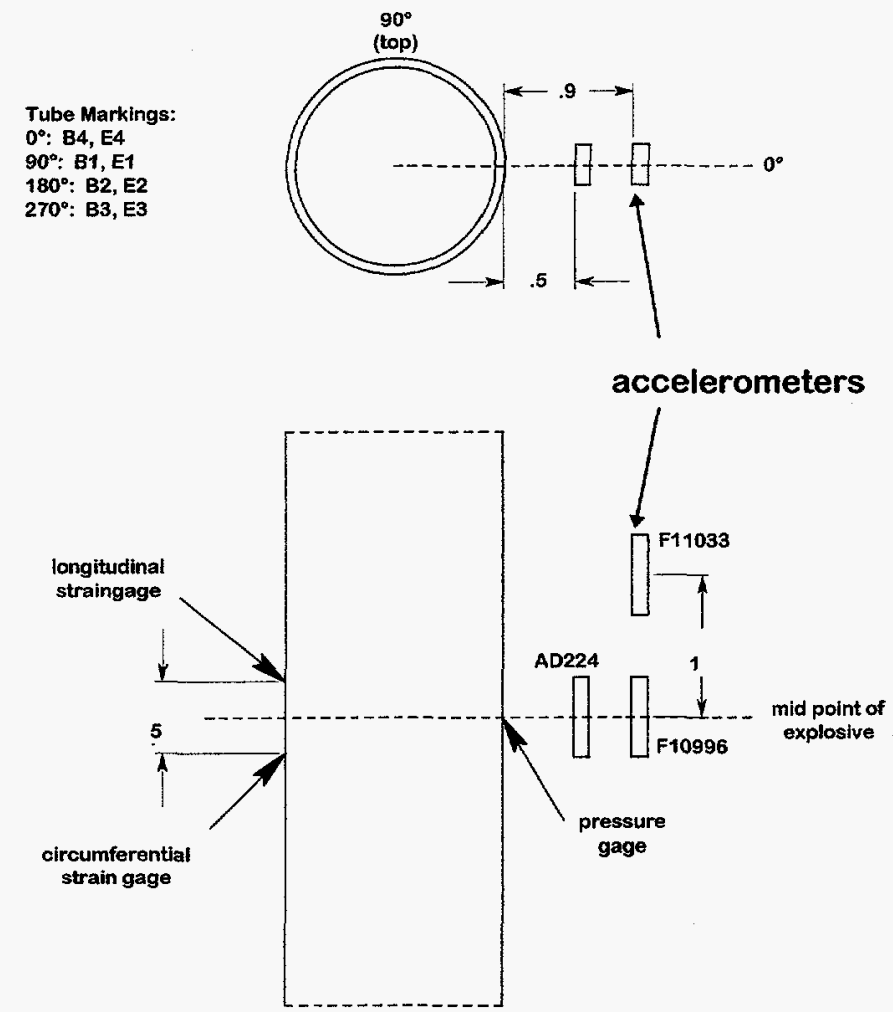

Figure 4.4. Transducer Locations for Test 1 and Test 2. 


\subsection{Test 2 - Static Test in Load Frame with 3 Gram Charge}

Following Test 1, the instrumented assembly was placed in a load frame as shown in Figure 4.5 and Figure 4.6. The soil was subjected to a $33135 \mathrm{lb}$ load with a hydraulic cylinder. This load was equivalent to a $60 \mathrm{psi}$ load which would occur at the depth of the tube if the container was subjected to $100 \mathrm{G}$ 's. During this loading, the soil compressed approximately 2.75 inches. This loading confirmed structural integrity of the soil container.

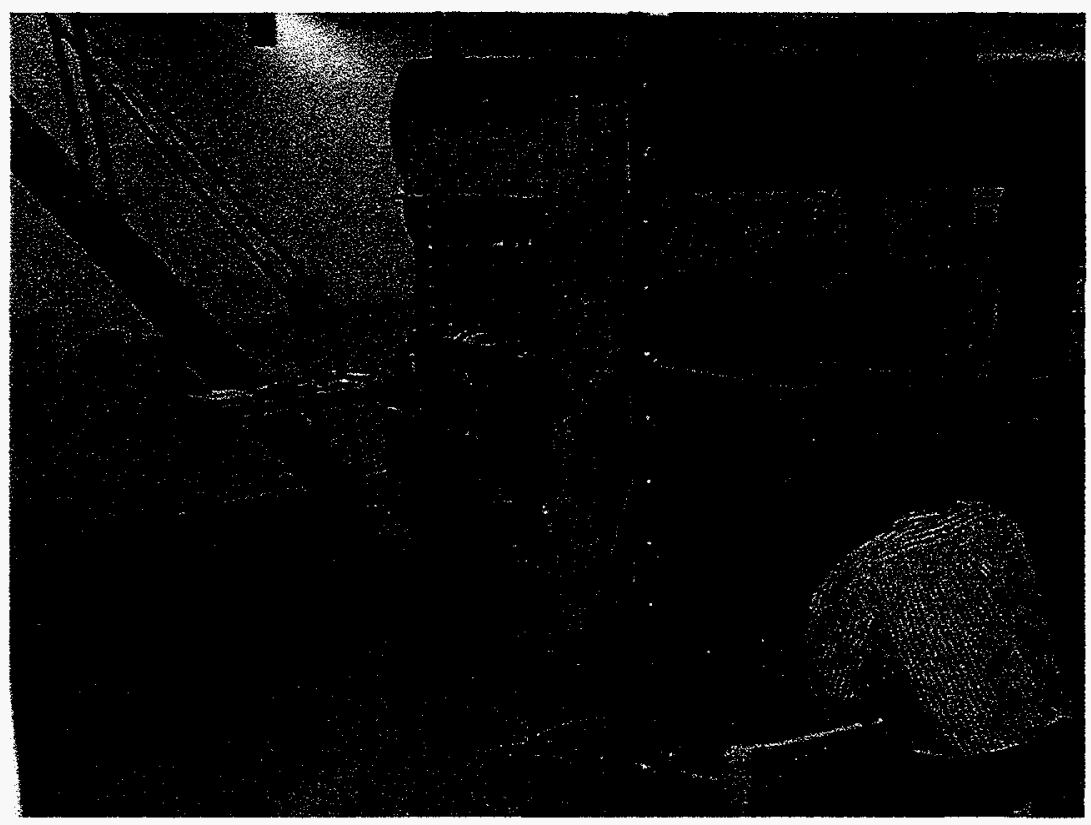

Figure 4.5. The instrumented assembly placed in a load frame.
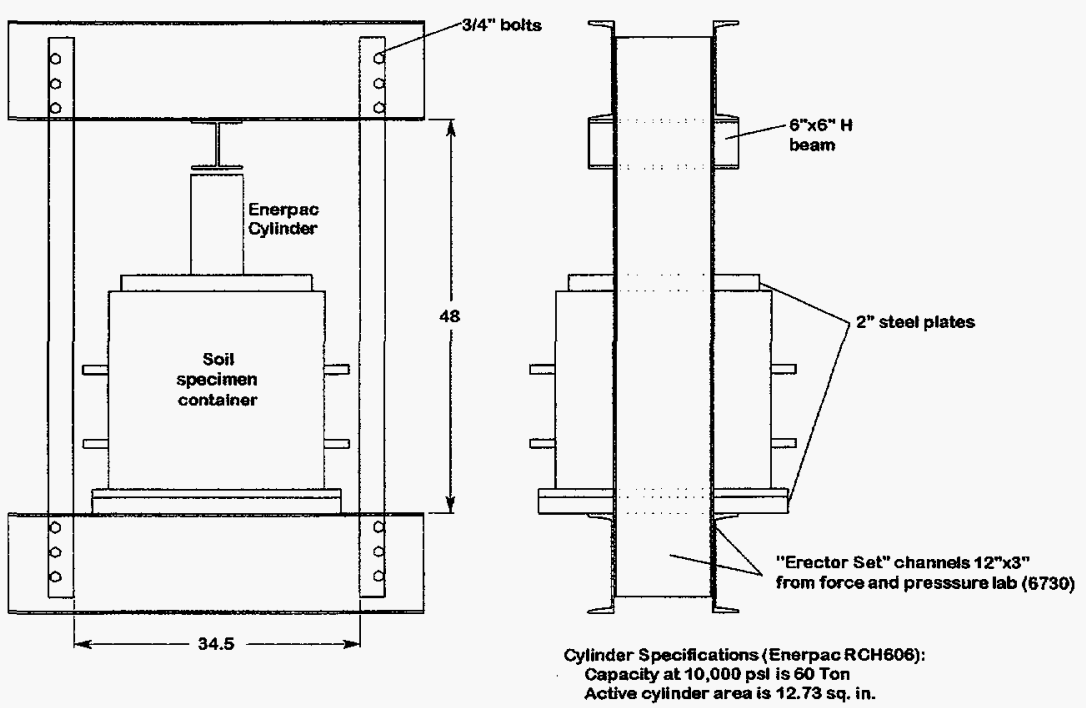

Figure 4.6. Load Frame Assembly. 
After the load was relieved, a 3 gram charge was installed into the center of the tube, the compressive load was reapplied, and the charge was detonated. Strain and accelerometer data were successfully measured (see Section 4.7). No pressure data was measured due to a failed transducer.

Before the soil was removed from the container to examine the effects of the explosive on the tube, the container was installed onto the centrifuge swing fixture. Two $100 \mathrm{G}$ centrifuge runs were conducted. On the first run, a detonator was fired to demonstrate proper control of the fireset through the slip rings while the centrifuge was operating. On the second run, a 3 gram charge assembly was placed into the tube, but not fired, to demonstrate the structural integrity of the explosive assembly during a $100 \mathrm{G}$ loading. Both runs were successful in demonstrating the required capability.

After these centrifuge runs, the soil, and aluminum tube were removed from the container. As shown in Figure 4.7, the center of the tube had been expanded by the action of the explosive charge, as was expected based on analytical predictions. However, the bottom portion of this expanded section also had a longitudinal tear, which was not expected. It was noted that the tube had a permanent bow in it from end to end. The center of the tube had deflected downward approximately 0.27 inches with respect to a straight line between the ends of the tube. Apparently, the friction between the end plates and the container had restricted the motion of the ends of the tube as the soil was being compressed (though the tube end moved downward 1.25 inches at the slip plate). This frictional force increased as the pressure in the soil increased. This restriction of the motion of the ends of the tube resulted in the soil pressure being greater on the top of the tube than on the bottom. Figure 4.8 illustrates this condition. The lower soil pressure on the bottom of the tube could explain the longitudinal tear in the bottom of the tube.

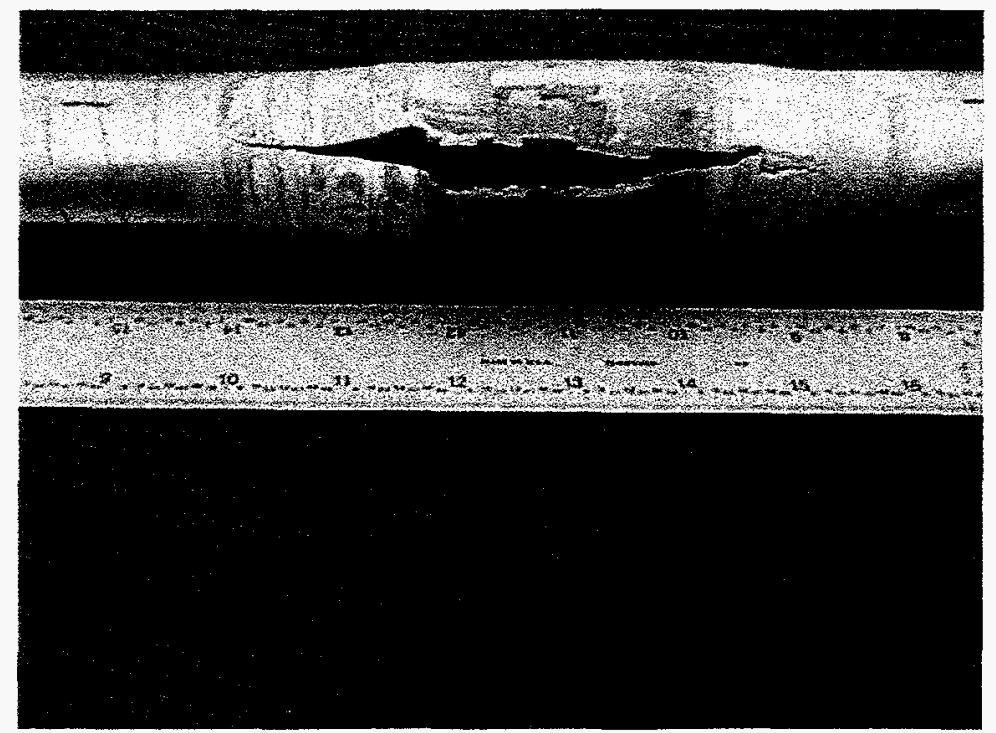

Figure 4.7. The center of the tube expanded by the action of the explosive charge. 


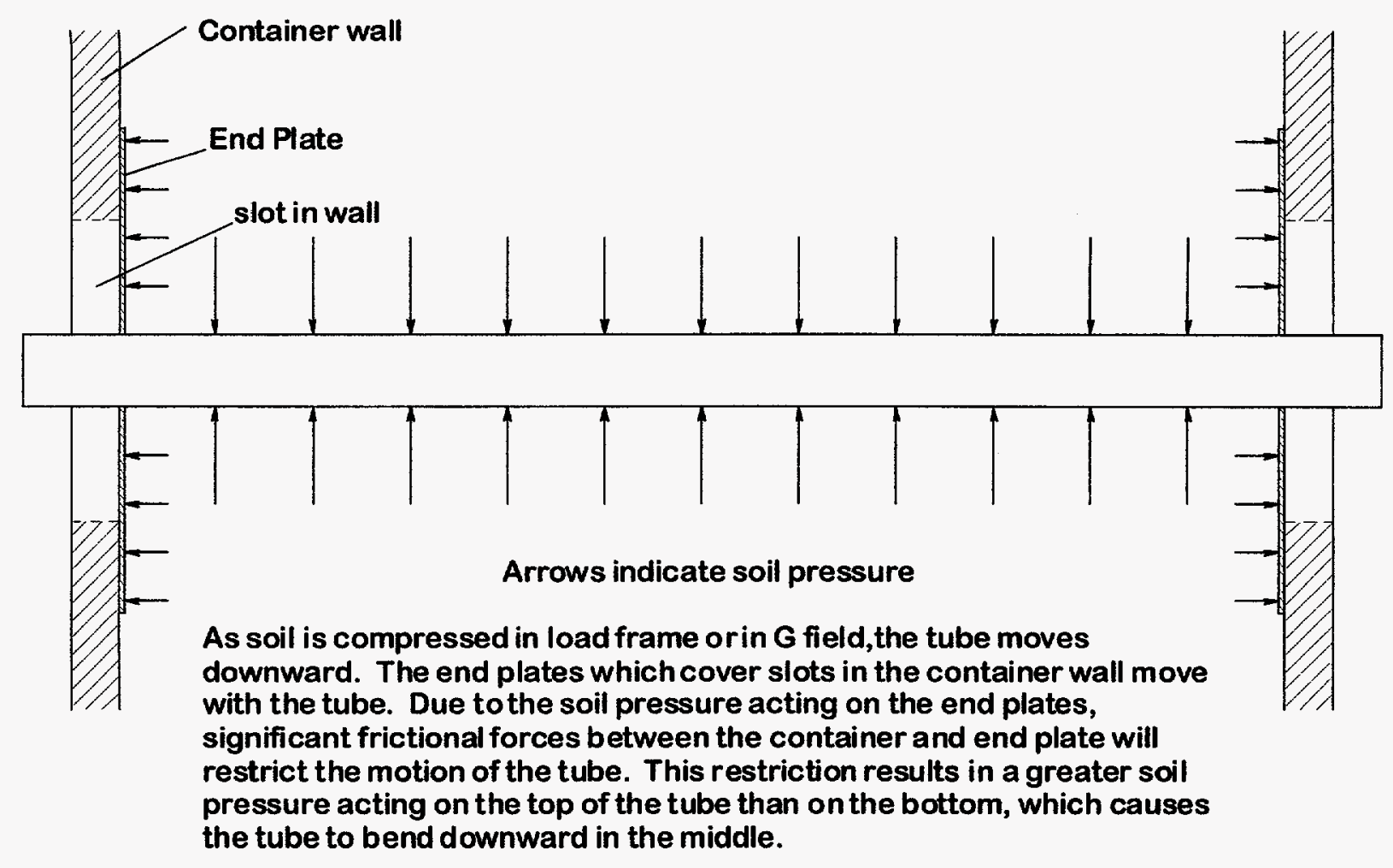

Figure 4.8. Explanation of Tube Bending.

\subsection{Test 3 - $100 \mathrm{G}$ Centrifuge Test with 3 Gram Charge}

The soil container was refilled, with two strain gages and a pressure gage on the tube, and five accelerometers in the soil. Figure 4.9 shows the location of the transducers for this assembly. The 1/8 inch aluminum end plates used in the previous assembly were replaced with Teflon plates to reduce the frictional constraints on the end of the tube. The instrumentation cables were routed with strain relief as previously described. This assembly was then attached to the centrifuge swing fixture, and a 3 gram charge was installed in the center of the tube. Next, a 100 $\mathrm{G}$ centrifuge run was conducted during which the explosive charge was detonated. Data were successfully recorded (see Section 4.7) for all transducers except for one accelerometer (F11033).

Following the test, the soil and tube were removed from the container. Figures 4.10 and 4.11 show the tube, which exhibited radial expansion, and longitudinal tearing as in the previous test. The middle of the tube was bent downward by about 0.25 inches as it was in the previous test. Apparently the Teflon end plates did not significantly reduce the end constraints on the tube. Again, the tearing is believed to have resulted from a lower soil pressure on the bottom of the tube. The tearing was not predicted by analytical model results. 


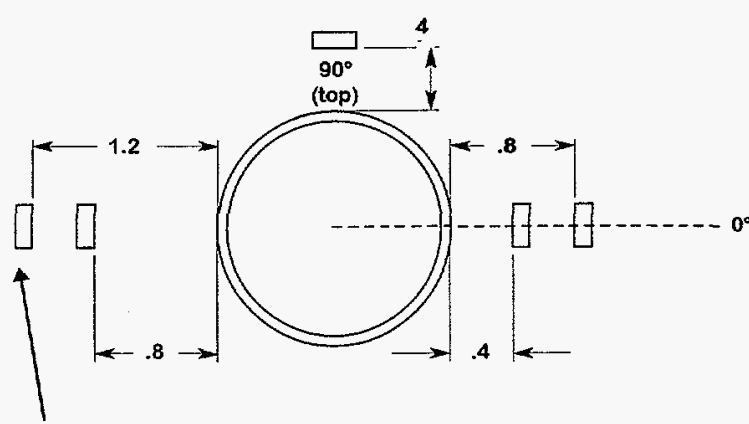

Tube Markings:

$0^{\circ}:$ A2, C2

$90^{\circ}: \mathrm{A1}, \mathrm{C1}$

$180^{\circ}: \mathrm{A4}, \mathrm{C4}$

270" $A 3, C 3$

accelerometers

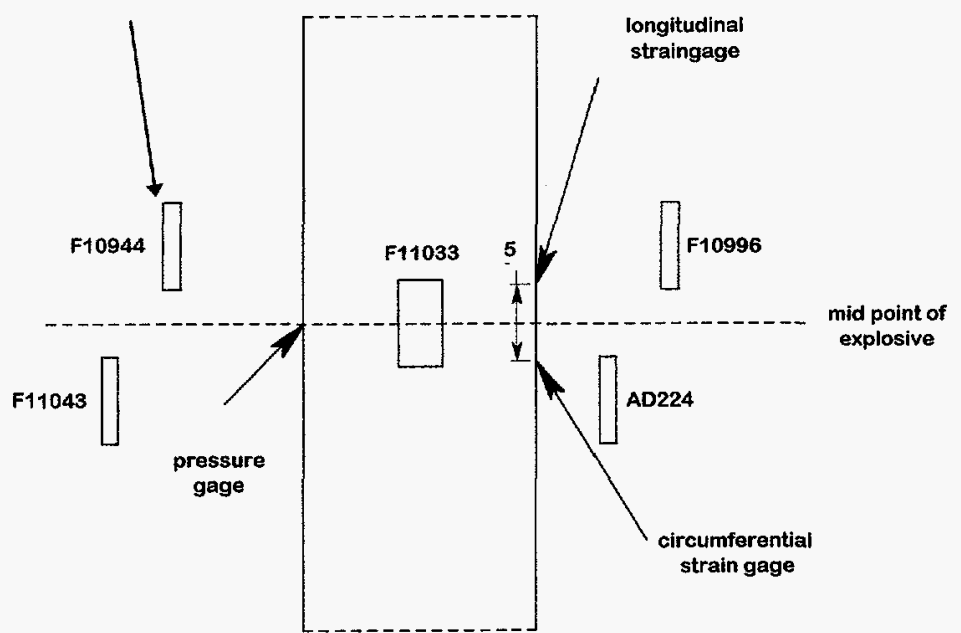

Figure 4.9. Transducer locations for Test 3.

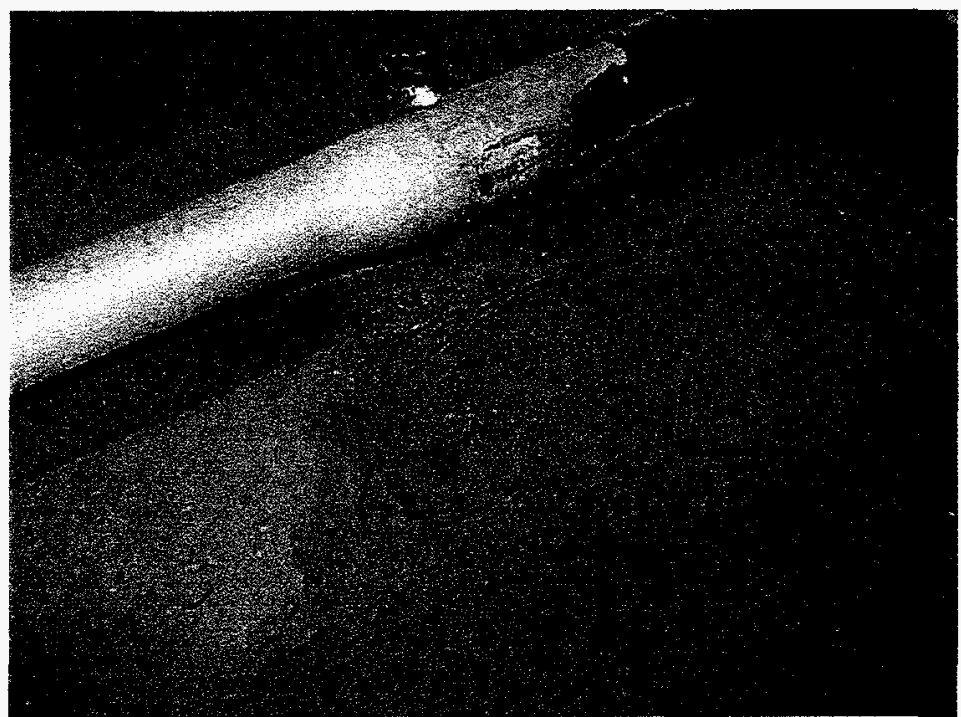

Figure 4.10. Radial expansion of the tube in Test 3. 


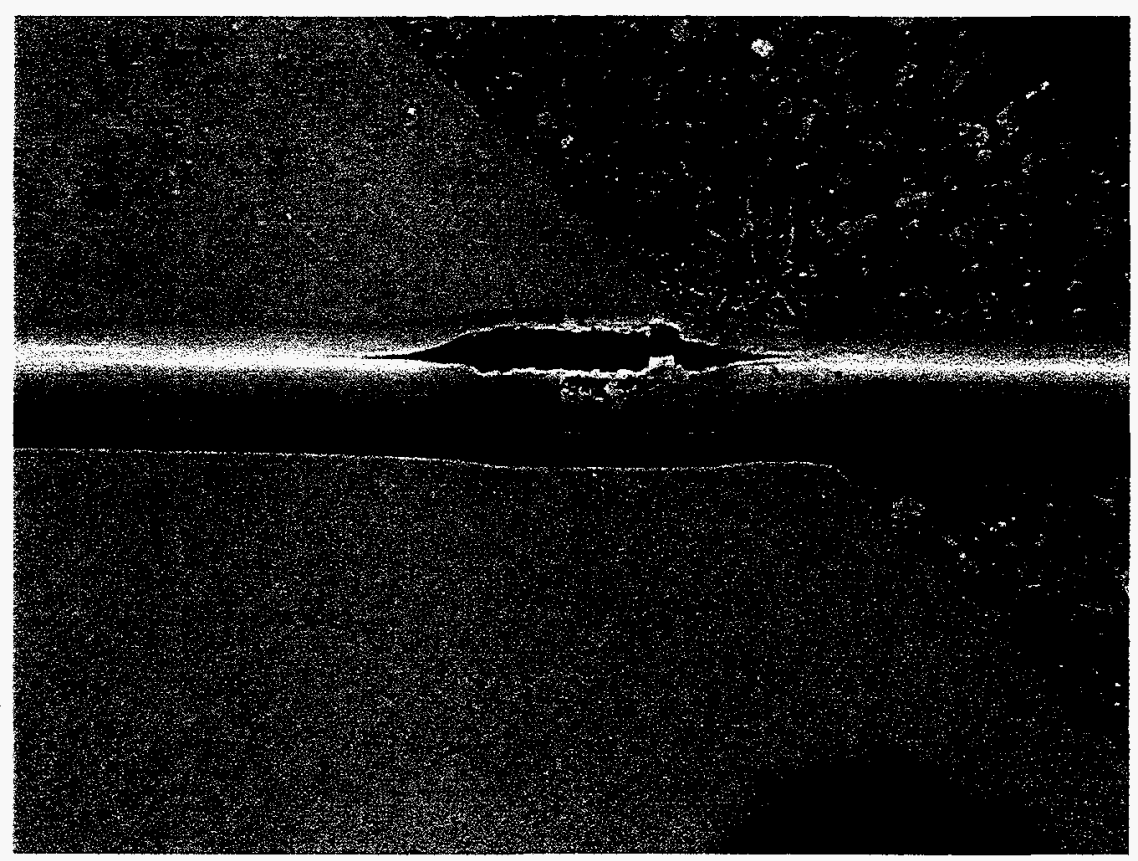

Figure 4.11. Longitudinal tearing of the tube in Test 3.

\subsection{Experimental Results and Analytical Model Predictions}

Figures 4.12, 4.13, and 4.14 show the measured Test 2 (static test in the load frame) data for acceleration, hoop strain, and longitudinal strain, respectively. Computer simulations were not performed for the Test 2 scenario.

Figures $4.15,4.16,4.17$, and 4.18 compare the measured Test 3 (dynamic test at $100 \mathrm{G}$ ) data for acceleration, hoop strain, longitudinal strain, and tube wall pressure, respectively, to that predicted by the computer simulation.

The measured accelerations shown were multiplied by a factor of 4 for the comparison with the analytical results shown in Figure 4.15. This factor was applied to the measured data because the analytical model does not include an accelerometer in the soil. The accelerometer is approximately 4 times heavier than the equivalent volume of soil, so the measured accelerations would be about 4 times lower than the analytically predicted soil accelerations. This correction assumes that the soil pressures are not significantly affected by the presence of accelerometer.

In both Test 2 (at 0.9 inches) and in Test 3 (at 0.8 inches), the measured peak acceleration were different by factors of 2-3 at transducers located close to each other. One possible reason for the difference was that the face of the transducer may have shifted from a normal axis (to the explosive) as the soil compacted prior to the detonation.

Another interesting point was that in Test 3, the measured acceleration close to the tube (at 0.4 inches) was greater than predicted by the computer model and that the measured acceleration 
father from the tube (at 1.2 inches) was less than predicted. This implied that the "damping" of the shock wave in the soil was greater than that predicted.

Figures 4.16 and 4.17 show that the measured tube hoop and longitudinal strain closely matched predicted values. Figure 4.18 shows that the measured tube wall pressure history also closely matched predicted values. As an aside, predicted soil pressure at an element next to the wall is also plotted in Figure 4.18. Note that the soil pressure is drastically decreased, due to the collapsing behavior exhibited by the soil.

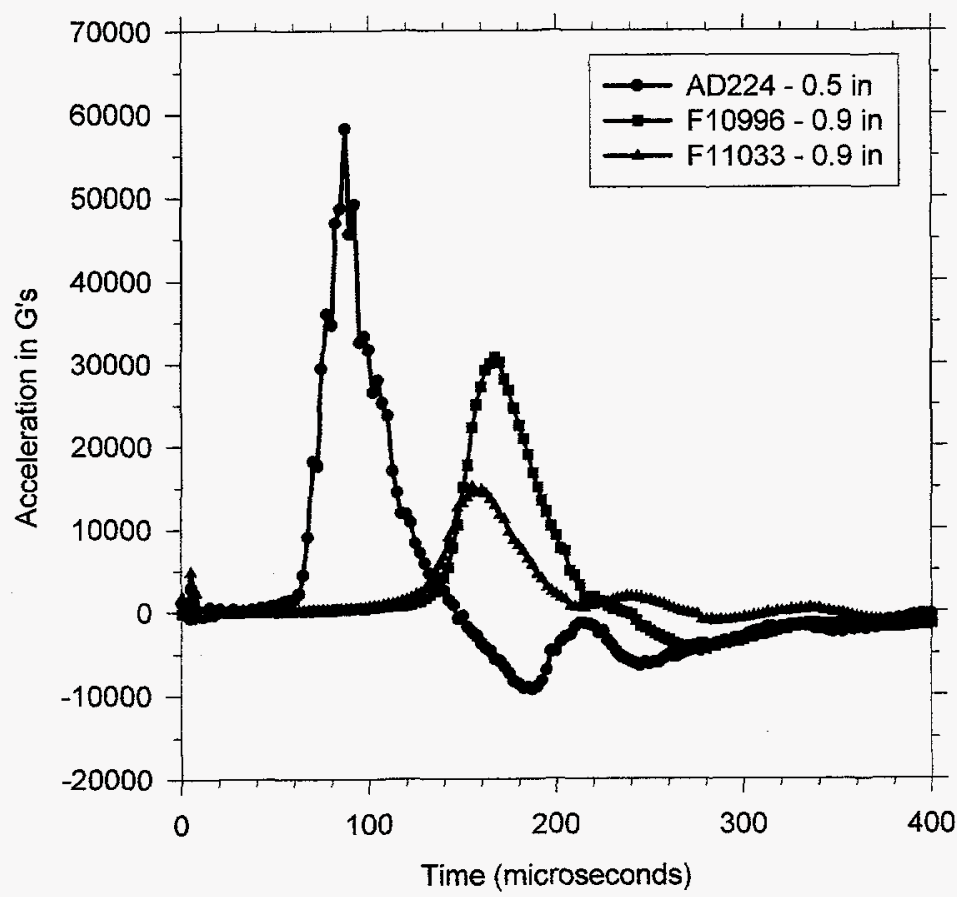

Figure 4.12. Acceleration versus time at various locations in Test 2. 


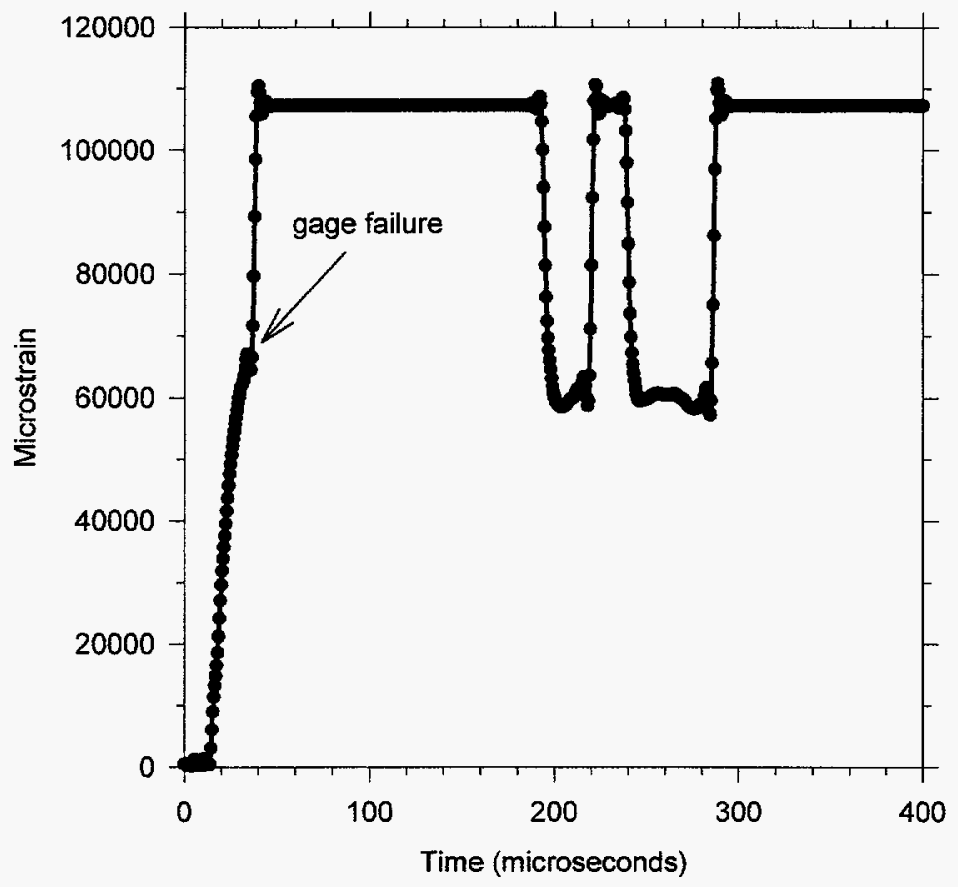

Figure 4.13. Hoop strain versus time in Test 2.

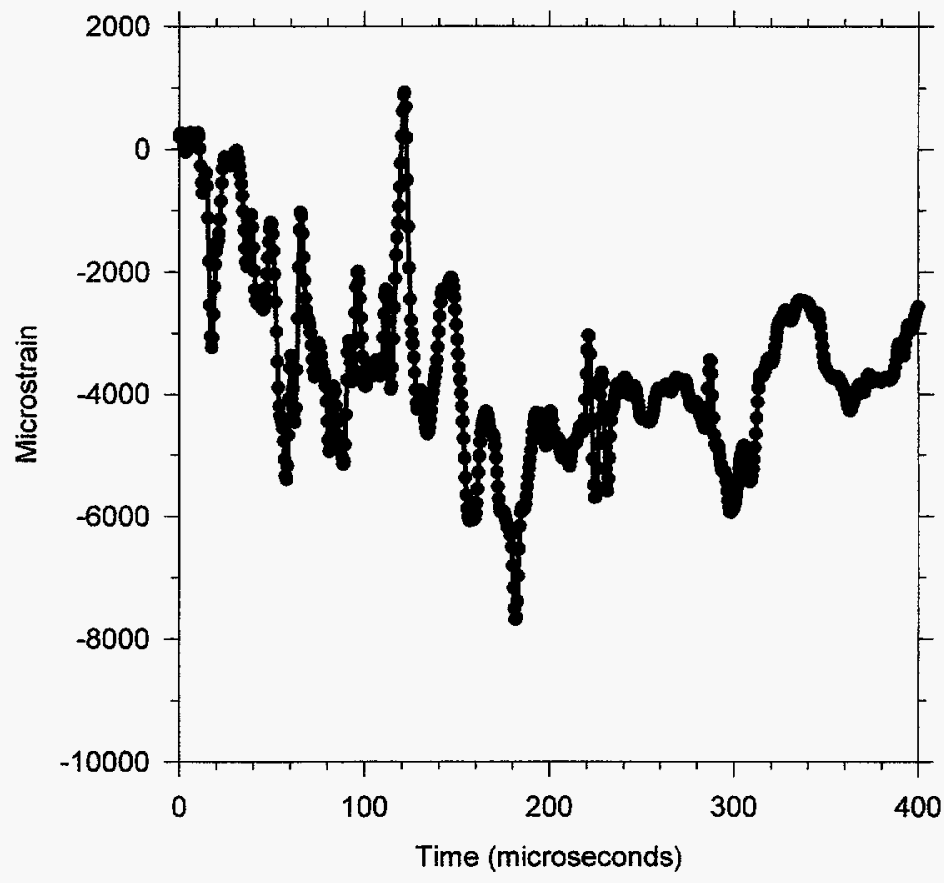

Figure 4.14. Longitudinal strain versus time in Test 2. 


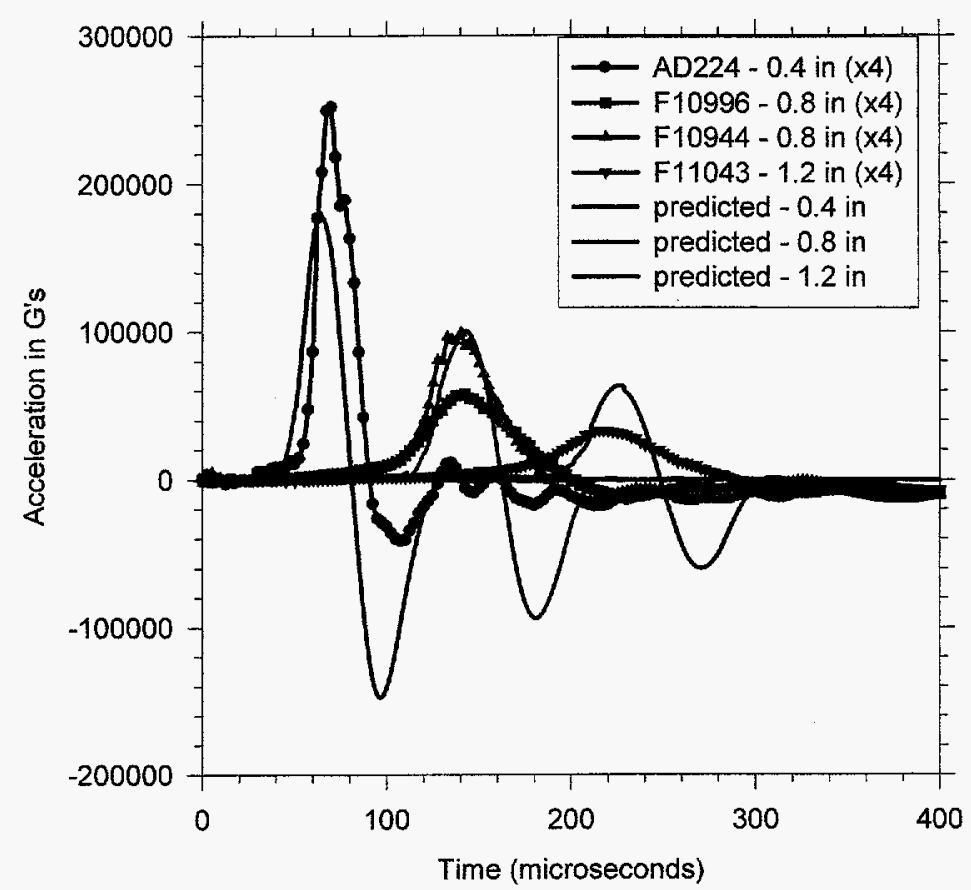

Figure 4.15. Acceleration versus time at various locations in Test 3.

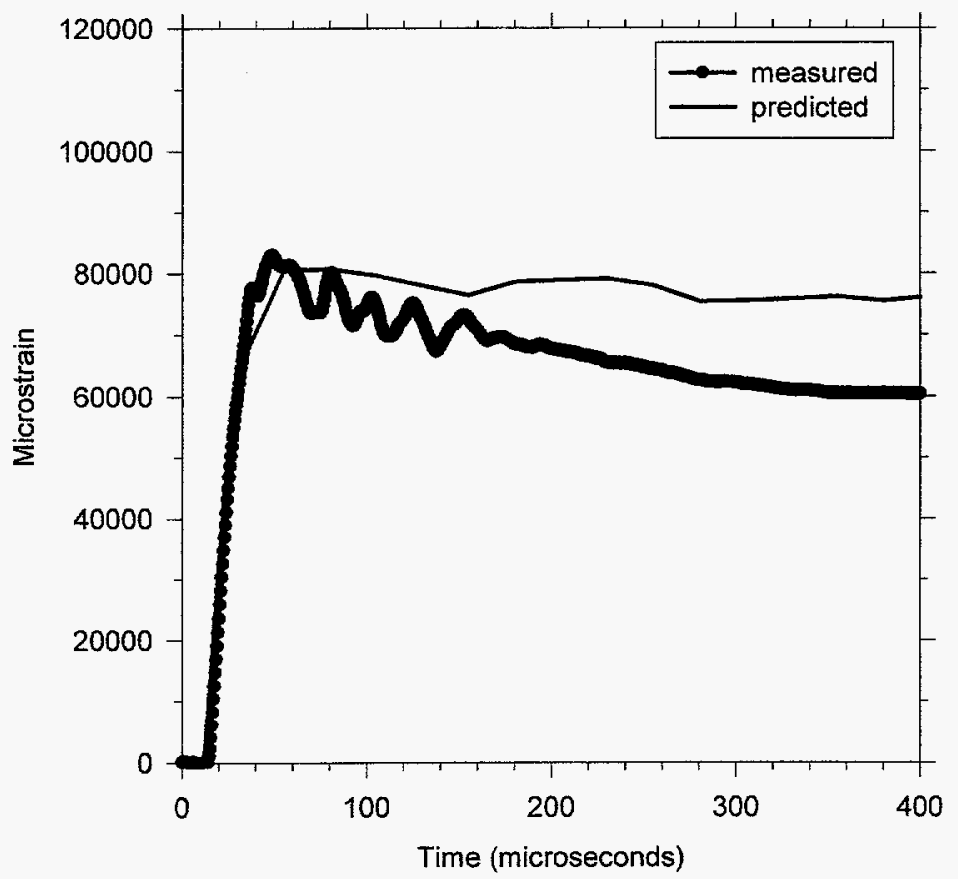

Figure 4.16. Hoop strain versus time in Test 3. 


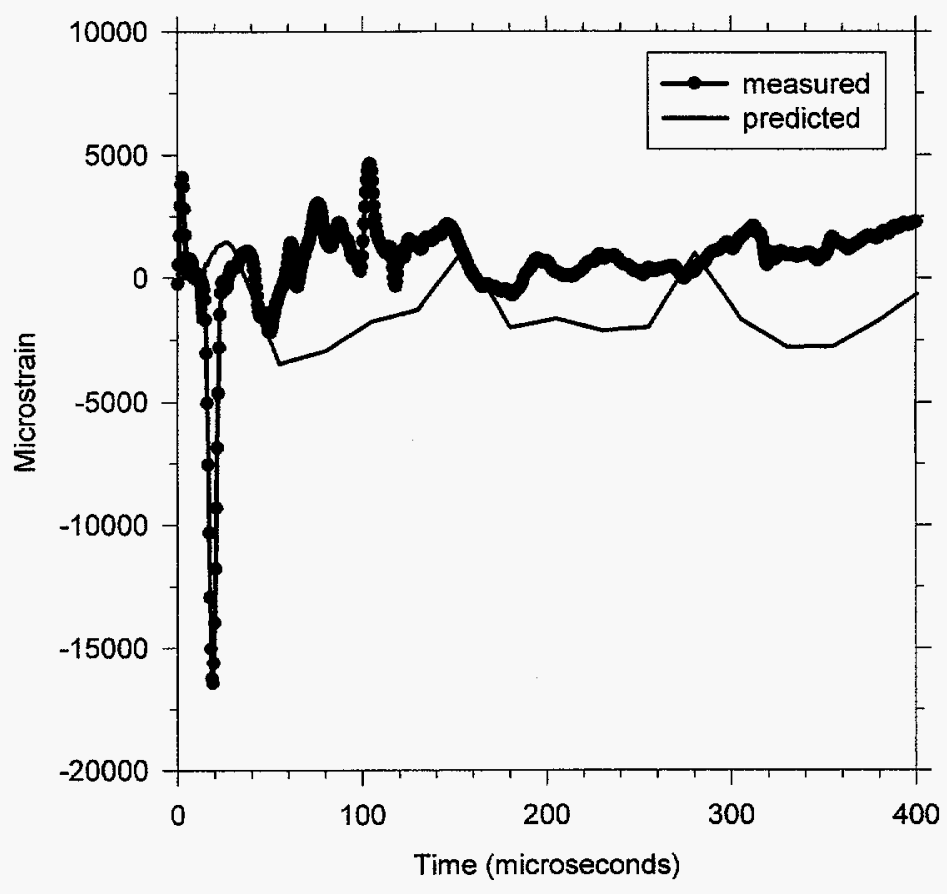

Figure 4.17. Longitudinal strain versus time in Test 3.

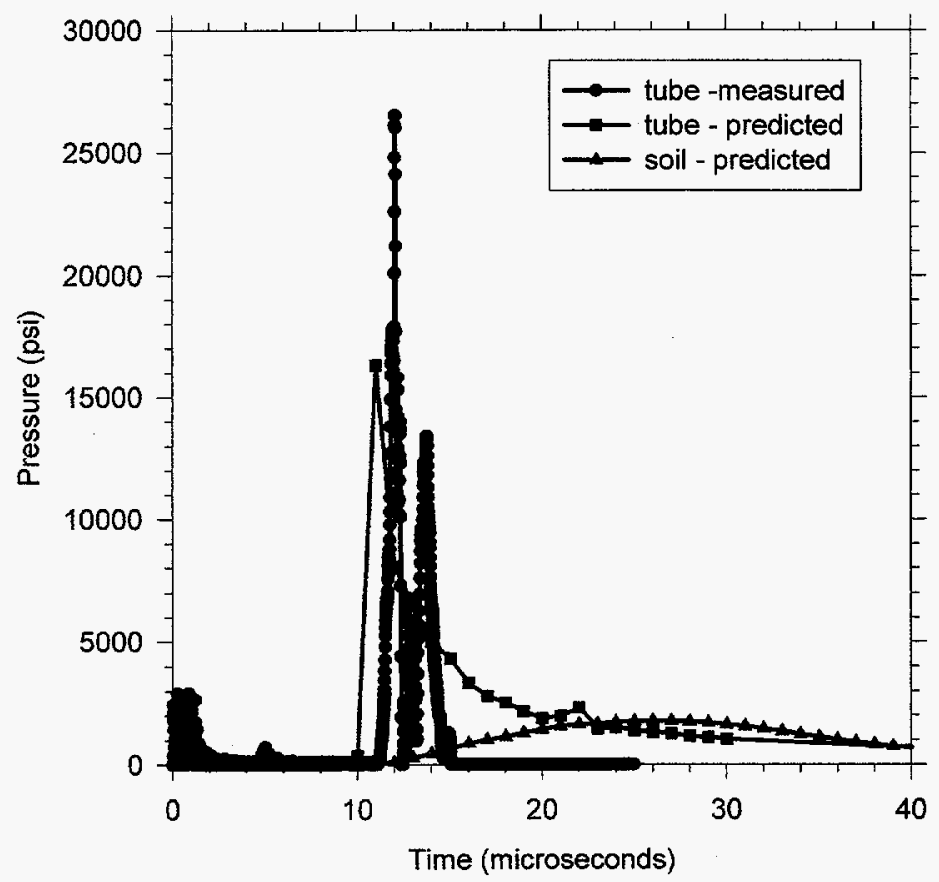

Figure 4.18. Wall pressure versus time in Test 3 


\subsection{CONCLUSIONS}

Expertise in the testing of explosive loading of scaled geotechnical structures, utilizing the unique capabilities of the Large Centrifuge Facility, was developed. Considerable dialog and cooperation between computational and experimental departments resulted in a successful program. Sandia's hydrocodes, dynamics codes, and soil models were key elements in the experimental design. Pretest numerical calculations were performed to assess the vulnerability of a metal-lined subterranean tunnel that was subjected to an explosive event. An experiment was performed that validated the following hypothesis: gravity scaling laws are valid for explosive loading of scaled structures in a steady-state centrifugal acceleration field. The experiment provided data to help evaluate the predictive capabilities of existing hydrocodes and structural dynamics codes developed at Sandia.

Most of the measured data compared very well with the pretest blind computer simulations. Discrepancies between observed and predicted soil acceleration magnitudes were explained by the different equivalent masses between the soil and the physical transducers (that were not explicitly modeled in the computer simulations). This error could be reduced by either modeling the instruments or by using transducers with similar mass properties for the media of interest.

The experimental techniques and core knowledge developed under this program can now be applied to more sophisticated problems. Realistic geotechnical structures, such as underground bunkers, tunnels and building foundations, and actual stress fields produced by the gravity load on the structure and/or any overlying strata can be tested. These stress fields may be reproduced on a scale model of the structure by proportionally increasing the gravity field through the use of a centrifuge. Further study would focus on both buried structures such as hardened storage bunkers or tunnels in rock mediums and also above ground structures. This technology can then be used to assess the vulnerability of various geotechnical structures to explosive loadings (such as car bombs in basement garages). Applications of this technology include assessing the effectiveness of earth penetrating weapons, evaluating the vulnerability of various structures, counter-terrorism, and model validation. 
This page intentionally left blank. 


\section{APPENDIX A}

THE SCALED TUNNEL MODEL HARDWARE

The tunnel model utilized 6061-T6 aluminum seamless tubing, manufactured by VAW. The tube had a nominal diameter of 1.5 inches outer diameter (OD) and a wall thickness of 0.083 inches. Detailed measurements on the pipe showed that the pipe was relatively round and the thickness was fairly uniform. Table 5.1 shows the measurements on sections of the tube used in two separate tests. Tube 2 was used in Test 2 (the static test) and tube 3 was used in Test 3 (the dynamic test). Each tube end was marked at 90 degree intervals and measured. The layout was such that E1 was opposite to B1 and so on.

Table A.1. Aluminum tube measurements

\begin{tabular}{|c|c|c|c|c|c|}
\hline $\begin{array}{c}\text { Tube 2 } \\
\text { Location }\end{array}$ & $\begin{array}{c}\text { Tube 2 } \\
\text { Diameter } \\
\text { (inches) }\end{array}$ & $\begin{array}{c}\text { Tube 2 } \\
\text { Wall Thickness } \\
\text { (inches) }\end{array}$ & $\begin{array}{c}\text { Tube 3 } \\
\text { Location }\end{array}$ & $\begin{array}{c}\text { Tube 3 } \\
\text { Diameter } \\
\text { (inches) }\end{array}$ & $\begin{array}{c}\text { Tube 3 } \\
\text { Wall Thickness } \\
\text { (inches) }\end{array}$ \\
\hline E1 \& E3 & 1.502 & & $\mathrm{~A} 1$ \& A3 & 1.503 & \\
\hline E2 \& E4 & 1.503 & & $\mathrm{~A} 2 \&$ A4 & 1.502 & \\
\hline B1 \& B3 & 1.503 & & $\mathrm{C} 1 \&$ C3 & 1.503 & \\
\hline B2 \& B4 & 1.502 & & $\mathrm{C} 2 \&$ C4 & 1.502 & \\
\hline E1 & & 0.086 & $\mathrm{~A} 1$ & & 0.088 \\
\hline E2 & & 0.088 & $\mathrm{~A} 2$ & & 0.088 \\
\hline E3 & & 0.088 & $\mathrm{~A} 3$ & & 0.087 \\
\hline E4 & & 0.088 & $\mathrm{~A} 4$ & & 0.0875 \\
\hline B1 & & 0.088 & $\mathrm{C} 1$ & & 0.089 \\
\hline B2 & & 0.088 & $\mathrm{C} 2$ & & 0.088 \\
\hline B3 & & 0.089 & $\mathrm{C} 3$ & & 0.087 \\
\hline B4 & & 0.087 & $\mathrm{C} 4$ & & 0.088 \\
\hline
\end{tabular}

The tube was stamped WWT 700/6F ASTM B210. The following engineering mechanical properties were obtained (Popov, 1990) and utilized in the computer simulation: density $0.1 \mathrm{lb} / \mathrm{in}^{3}$, elastic modulus $10 \times 10^{6} \mathrm{psi}$, yield strength (tension) $35 \mathrm{ksi}$, and ultimate strength (tension) $38 \mathrm{ksi}$. 
This page intentionally left blank. 


\section{APPENDIX B THE SOIL CONTAINER}

The soil container (Figure B.1) was designed to contain 8 cubic feet of conditioned soil that is accelerated to a steady state level of $100 \mathrm{G}$. The resulting design was a square box that measured 26 inches high, 26 inches wide and 25 inches tall. The internal dimensions were 24 x $24 \times 24$ inches and the wall thickness was one inch. The soil had a density of $1.393 \mathrm{~g} / \mathrm{cc}$ $\left(86.95 \mathrm{lb} / \mathrm{ft}^{3}\right)$ which results in a maximum pressure of 120 psi acting over the base of the container. At $100 \mathrm{G}$, the resultant loading on each side plate was $34795 \mathrm{lb}$ and the resultant loading on the bottom plate was $69560 \mathrm{lb}$. These are the maximum loads based on the conservative assumption that the soil behaves as a fluid. Based on the data from the explosive model, the transient loading from the explosive charge is small in comparison with the steady-state loading; therefore, only the steady-state loading was considered in the analyses described below.

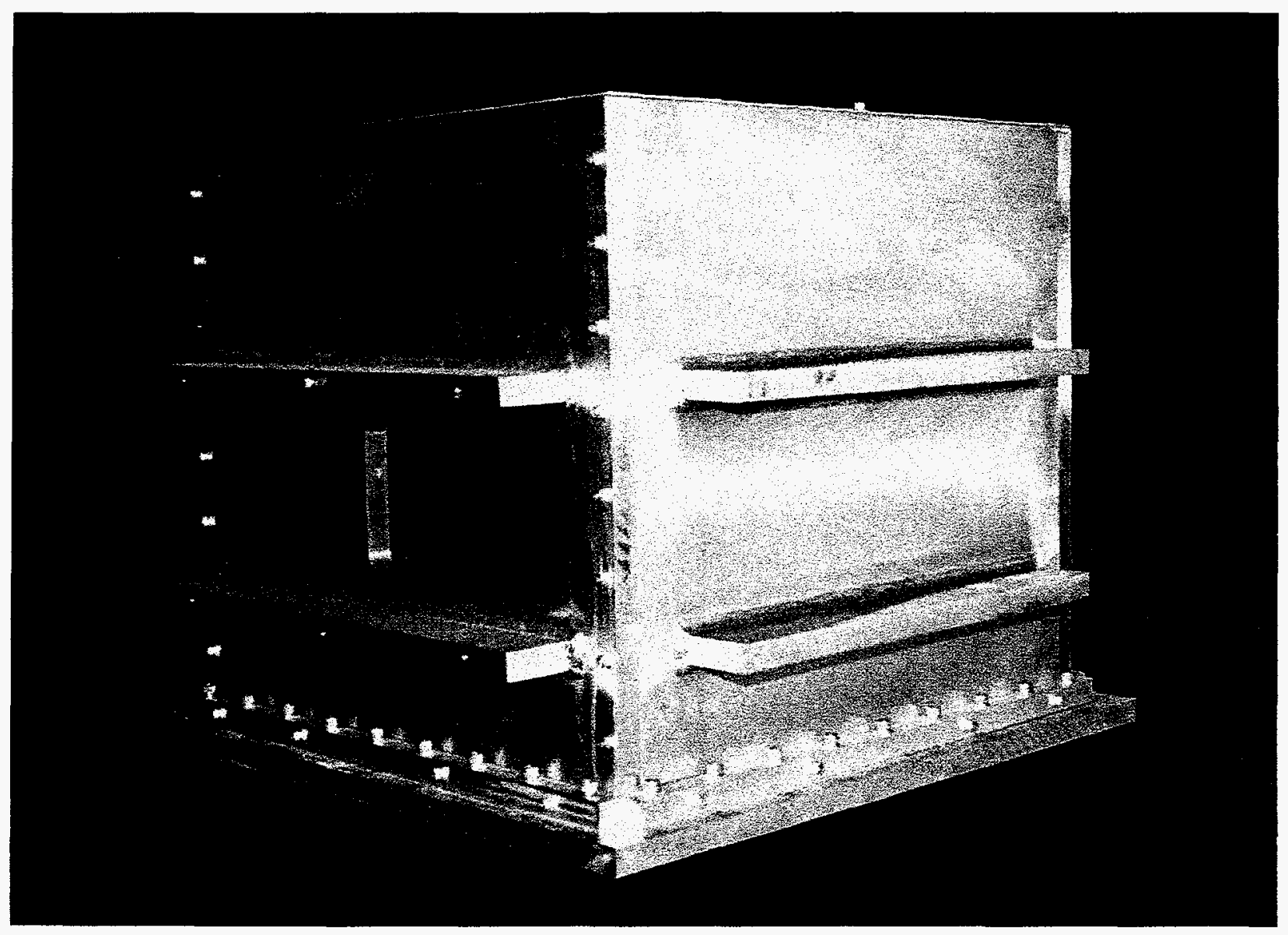

Figure B.1. The soil container.

The design on the container walls assumed that each wall was supported by the bolts on the vertical edges only. This resulted in two sides with through holes and two sides with threaded inserts. Sixteen 5/16-18 socket head cap screws with heavy duty keenserts were used. Keenserts were chosen because of their superior wear characteristics with repeated 
assembly/disassembly procedures as compared to tapped aluminum holes. In addition, the Keenserts used a larger diameter thread size which has a higher pull-out strength. The 6061T6 aluminum has a shear strength of 30000 psi and a KNH 518 keensert has a pull out-force of $10052 \mathrm{lb}$. This yielded a total load capacity of $160832 \mathrm{lb}$ for a plate that is bolted to the inserts. The tensile stress on each $5 / 16$ screw for the $34795 \mathrm{lb}$ load was 47900 psi. Each screw would be subjected to shear and tensile stresses, but the combined loading did not approach the yield strength of the screws. Grade 8 socket head cap screws have a tensile strength of $150 \mathrm{ksi}$ and a yield strength of $130 \mathrm{ksi}$, well within the maximum load capacity. A wall thickness of at least 1 inch was required due to the 0.5 inch diameter hole required by the keensert.

Although the panels were 1 inch thick, the loading would cause significant bending in the plates; therefore, reinforcing flanges were welded to the plates for added stiffness. The analysis split each plate into three horizontal T-beams ( 8 inches wide and 26 inches long). The bottom panel has a maximum and minimum load pressure of 120 psi and 80 psi. The average loading was $800 \mathrm{lb} /$ inch along the top of the T-beam. This produced a tensile stress at the outer edge of a $3 \times 1$ inch flange of 22963 psi. The weld at this section can be subjected to a shear loading of $5176 \mathrm{lb} /$ inch. A $1 / 2$ inch fillet weld was specified for the flange; however, the weld material was only rated for 0.3 times the yield strength of the parent metal, 6061-T6 aluminum. This resulted in a shear loading capacity of $10500 \mathrm{lb} / \mathrm{inch}$ for the weld and a safety factor of 2.03 .

The middle 8 inch section was subjected to a loading $480 \mathrm{lb} /$ inch and would be subjected to less stress at the weld and flange. However, an identical flange with $1 / 2$ inch fillet welds was specified in order to mount a blast shield at the end of the tube. The top 8 inch section was analyzed but the loading did not warrant another flange.

In order to place the aluminum tube into the container, two slots had to be machined into opposite sides of the container. The slot width was oversized by 0.1 inch to allow the 1.5 inch diameter tube to move up and down as the soil was compressed due to the $G$ loading. The slot was machined such that the tube could move a total of 4.5 inches between centers. A thin aluminum slip plate was used to cover the full length of the slot so that the dirt did not spill out. The aluminum slip plate and the tube were expected to move downward with the soil as the soil compressed under the increased G load.

Each side plate was bolted to the base using a $1 \times 1$ inch aluminum bar as a footer. The footer was bolted to the base with nine 3/8-16 socket head cap screws (SHCS) and the side plate was attached to this footer using four 5/16-18 SHCS. Since the side plate was compressed onto the footer, these bolts were not be subjected to any tensile loading.

The fixture was clamped to the geotechnic swing arm using four clamps. These clamps were primarily used to position and stabilize the fixture under static conditions. Under test conditions, the increased normal force due to the centripetal acceleration would provide sufficient frictional forces to prevent any motion of the test fixture relative to the swing arm. 
In order to perform a preliminary proof test, the fixture was assembled in a test configuration and placed in a load frame. The aluminum tube was placed into the fixture and the fixture was filled with soil. A 23.5 inch square plate ( 2 inch thick) was placed on top of the soil and loaded to simulate the conditions at the tube depth due to $100 \mathrm{G}$. A charge was placed into the tube and detonated (Figure B.2). The test fixture was unaffected by the explosive and was not deformed in any way. This test is discussed in detail in Section 4.5. The test did show that the dynamic pressure due to the explosive detonation was minor and that the fixture could withstand the combined steady-state and dynamic soil loads.

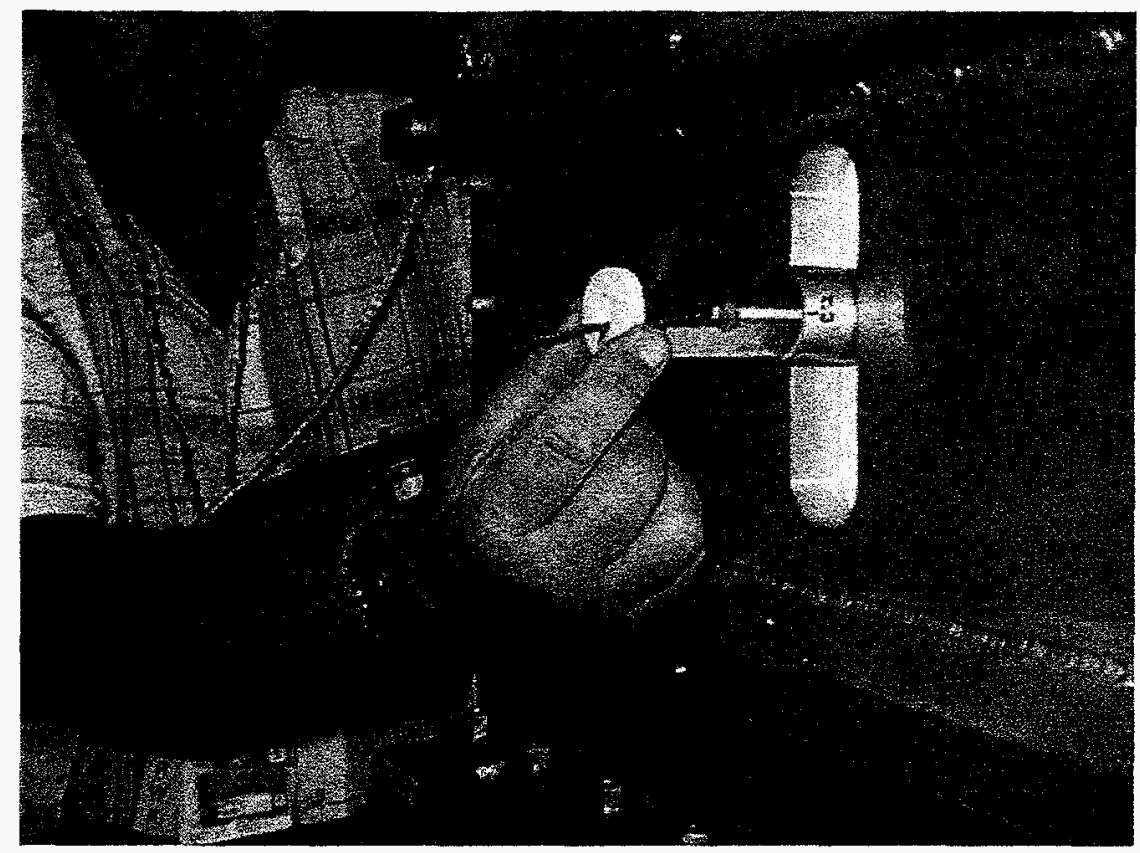

Figure B.2. Inserting an explosive charge into an assembled test fixture. 
This page intentionally left blank. 


\section{APPENDIX C MANUFACTURED SOIL AND EXPERIMENT ASSEMBLY}

The soil used for this experiment was a sand/clay mixture indigenous to Albuquerque, NM (Figure C.1). The soil was extracted from remnants of soil collected for an earlier Sandia project "Fuel Dispersal in High-Speed Aircraft/Soil Impact Scenarios" (Tieszen and Attaway, 1996). This soil was used because it was already characterized, well understood by analysts, and ample supplies remained.

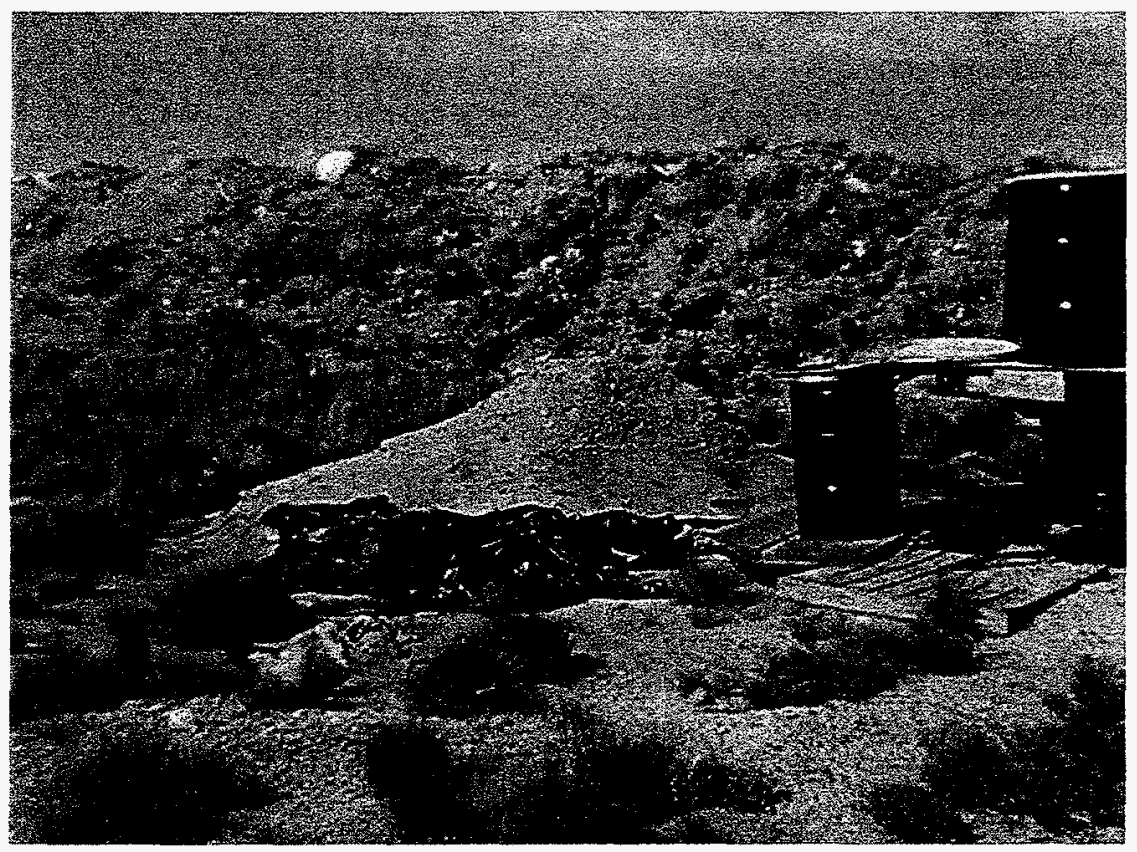

Figure C.1. The Albuquerque sand/clay soil mixture.

The soil was sieved through a No. 8 screen (Figure C.2) and then placed into six 55 gallon drums (Figure C.3), each one-third full to allow for mixing. The soil-drums were mixed end-over-end five times, then laid on their sides and rolled for ten revolutions. Soil moisture content measurements were made in accordance with ASTM D2216-92 for each soil drum (Figure C.4). Table C. 1 shows the data used to determine the initial moisture content of each of the six drums. Moisture was added to bring the moisture to $6.3 \%+/-0.6 \%$ of the soil mass. The individual drums were re-mixed with the mixing procedure indicated above.

The pre-mixed moist soil was laid into the soil fixture using 1-inch uncompressed lifts. Figure C. 5 shows a $10.2 \mathrm{~cm}$ diameter aluminum roller was used to roll the soil. The soil was rolled along parallel paths in one direction, then in the perpendicular direction, and finally in the initial direction again. The soil was hand packed in close proximity to the center-tube since the geometry of the setup precluded the use of the roller. A total of 34 lifts were required to fill the two foot deep soil container. 


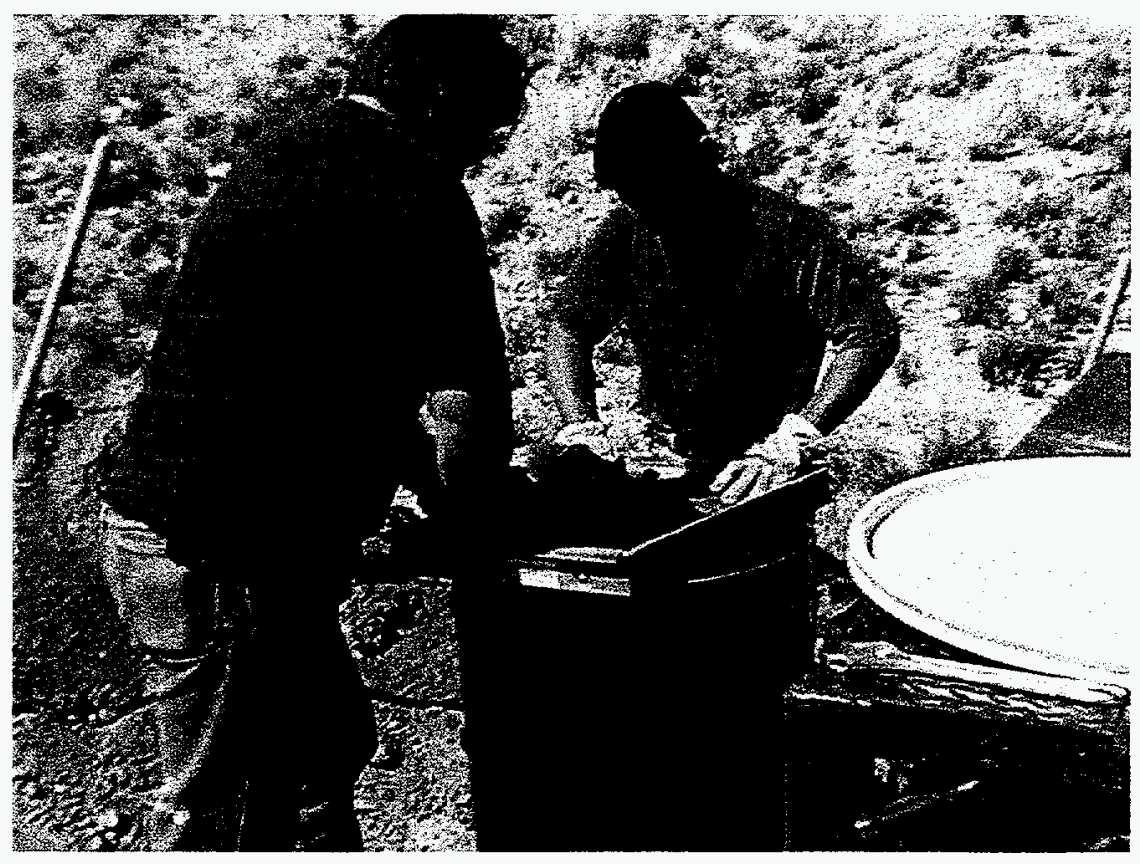

Figure C.2. Sieving the soil with a \#8 screen.

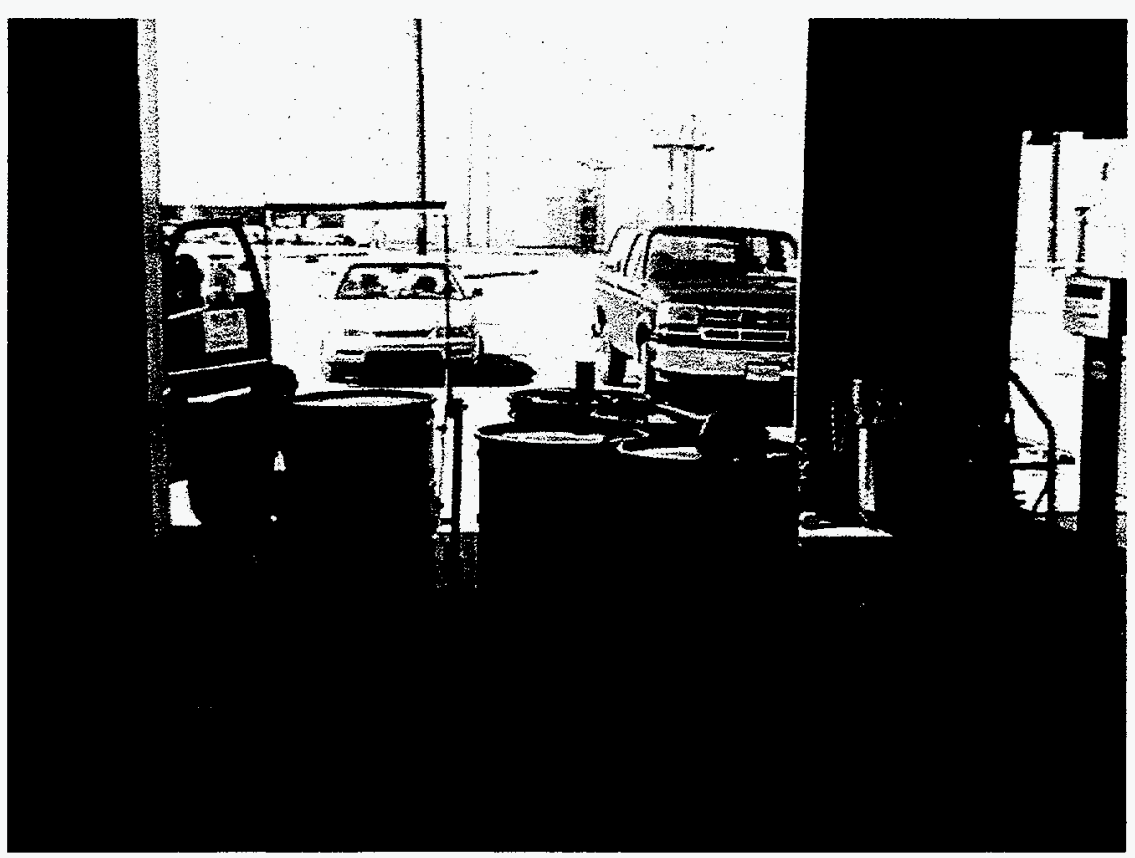

Figure C.3. Partial filling the drums with sieved soil. 


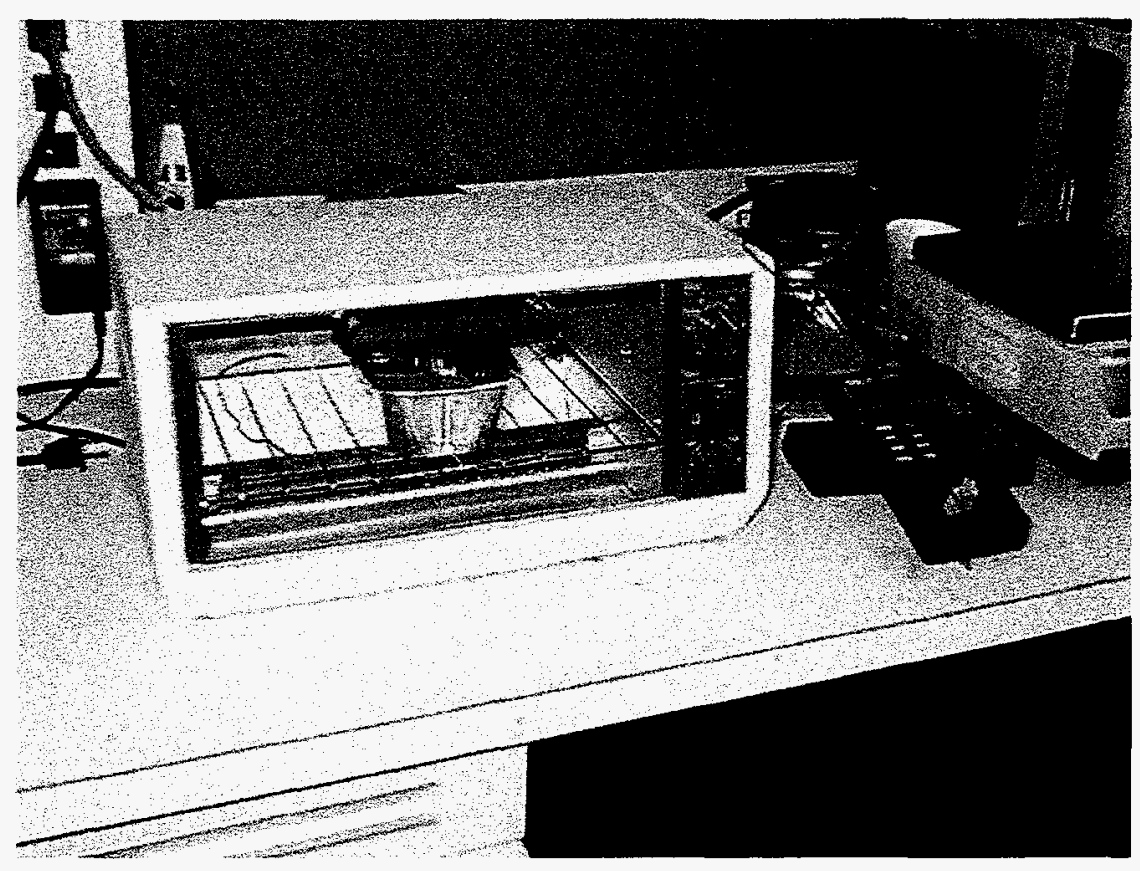

Figure C.4. Performing the soil moisture content analysis.

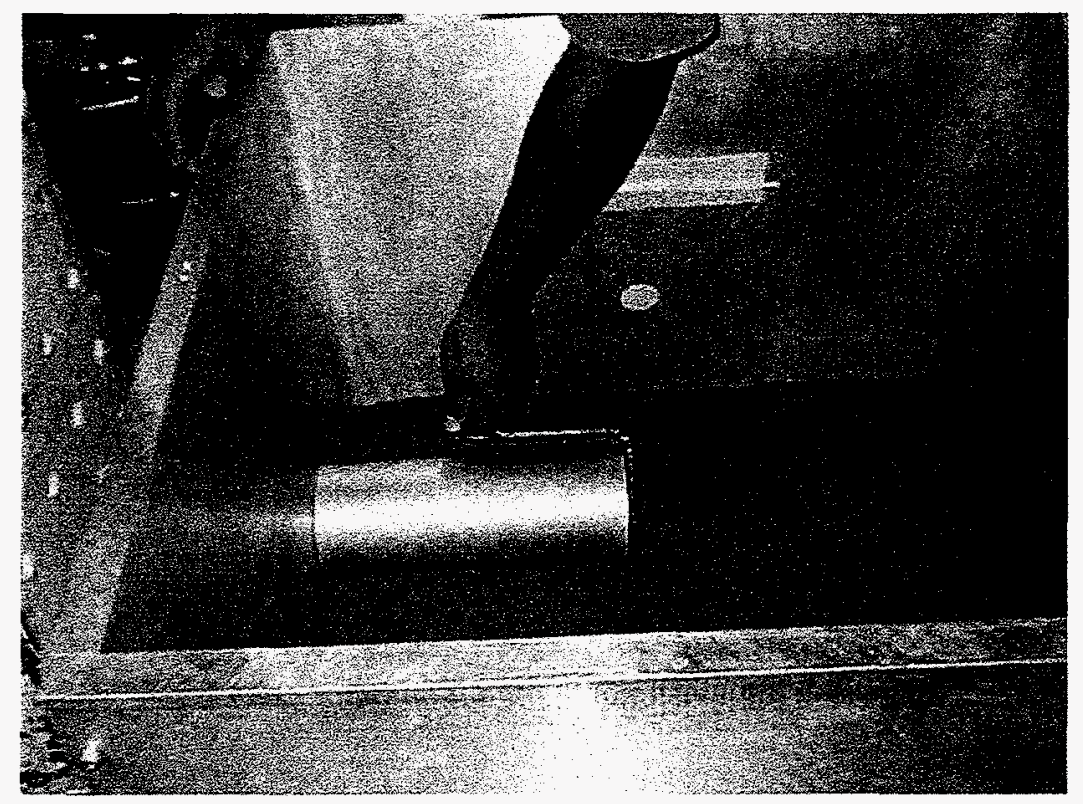

Figure C.5. Rolling the soil inside the explosive container.

Every effort was made to keep the soil drums and the soil fixture closed to prevent moisture evaporation. Table C.1 also presents moisture content measurements that were made following both the static press test (Test 2) and the centrifuge test (Test 3). The moisture mass was measured to be about $6.5 \%$ of the soil mass for both post test moisture content measurements. 
The post-static press test (in the load frame) soil sample was collected from approximately 4 inches from the top of the soil in the soil fixture and the post-centrifuge test soil sample was taken near the center tube.

The following equipment was used in determination of the soil moisture content: Mettler PC 8000 Scale, V953507, (0.1g readout), Black N Decker Toaster Oven, Model TRO400, Fluke Model 52 Thermometer, S/N 494033, Toledo Model 8140 Large Scale, S/N 4165838-4WN, (0.1 lb readout). The soil samples were heated until no further reduction in weight occurred. Note that the oven maintained a constant temperature of $230^{\circ} \mathrm{F} \pm 9^{\circ} \mathrm{F}$.

The following calculation was used to determine the soil moisture content (W\%)

$$
W \%=\frac{M_{T W S}-M_{T D S}}{M_{T D S}-M_{T}} \times 100
$$

where,

$\mathrm{M}_{\mathrm{TwS}}=\quad$ mass of tin and wet sample (g)

$\mathrm{M}_{\mathrm{TDS}}=\quad$ mass of tin and dry sample $(\mathrm{g})$

$\mathrm{M}_{\mathrm{T}}=$ mass of drying tin (g).

Table C.1. Soil moisture content data

\begin{tabular}{|c|c|c|c|c|c|c|}
\hline Drum & $\begin{array}{c}\text { Wet Soil Weight } \\
(\mathrm{lb})\end{array}$ & $\begin{array}{c}\mathrm{M}_{\text {TwS }} \\
(\mathrm{g})\end{array}$ & $\begin{array}{c}\text { Time Heated } \\
(\mathrm{min})\end{array}$ & $\begin{array}{c}\mathrm{M}_{\text {TDS }} \\
(\mathrm{g})\end{array}$ & W\% & $\begin{array}{c}\text { Water Added } \\
(\mathrm{g})\end{array}$ \\
\hline 1 & 193.5 & 111.1 & 55 & 104.8 & 6.5 & 0 \\
\hline 2 & 207.4 & 109.7 & 45 & 104.2 & 5.7 & 530.7 \\
\hline 3 & 156.0 & 111.5 & 45 & 106.1 & 5.5 & 535.1 \\
\hline 4 & 205.5 & 110.2 & 45 & 105.4 & 4.9 & 1241.0 \\
\hline 5 & 155.7 & 110.9 & 45 & 105.9 & 5.1 & 802.8 \\
\hline 6 & 205.3 & 110.6 & 50 & 105.7 & 5.0 & 1152.0 \\
\hline Static & na & 111.7 & 45 & 105.4 & 6.45 & na \\
\hline Centrifuge & na & 111.1 & 45 & 104.8 & 6.4 & na \\
\hline
\end{tabular}

General Weights:

Weight of empty 55 gal drums (w/o lid): $\quad 35.7 \mathrm{lb}$

Weight of empty sample tin: $\quad 7.8 \mathrm{~g}$ 


\section{APPENDIX D THE EXPLOSIVE SYSTEM}

\section{D.1 Introduction}

Computer simulations (described in Section 3) determined the sizing and configuration of the explosive system. Explosive pellets supplied by the Explosive Components Facility (ECF) were expected to produce the desired effects. The explosive charges were built and proof tested at the ECF. The following describes the design and performance of the explosive system.

\section{D.2 The Explosive System}

The explosive system consisted of 15 pressed pellets of 9407 explosive, each with a density of $1.62 \mathrm{gm} / \mathrm{cm}^{3}$. The length of each pellet was 0.250 inch and the diameter was 0.1875 inches. The pellets were glued "end to end" for a total charge length of 3.75 inches and were contained in a tube made of 0.001 inch thick Mylar (for strength). An RP-2 detonator was placed at one end to initiate the pellets.

The explosive system was fabricated at the ECF. A 4 inch long phenolic tube $(0.200$ inch ID) was first lined with 0.001 inch thick Mylar to keep the glue from adhering to the phenolic tube and to provide strength to the final pellet cylinder. A thin layer of "Barco Bond" 15-minute epoxy was smeared on each end of the pellets. The pellets were then stacked in the phenolic tube. This tube and explosive assembly was then placed in a small press for 24 hours to allow the glue to cure. After curing, the pellet train was pushed out of the phenolic tube with the 0.001 inch thick Mylar attached. A 0.250 inch long phenolic cylinder was glued to one end of the explosive train to provide support and alignment for the RP-2 detonator. Figure D.1 is a diagram that shows the gluing and pressing process.

Figure D.2 is a sketch of the finished charge. A total of 16 pellets were used for the charges that were tested at the centrifuge. The charge was positioned on a cardboard pedestal that was designed for supporting the charge in the tube. Figure D.3 shows a photo of the explosive charge attached to the pedestal. The rigid foam discs at the end of the pedestal were designed to keep the charge centered in the tube during the centrifuge tests. 


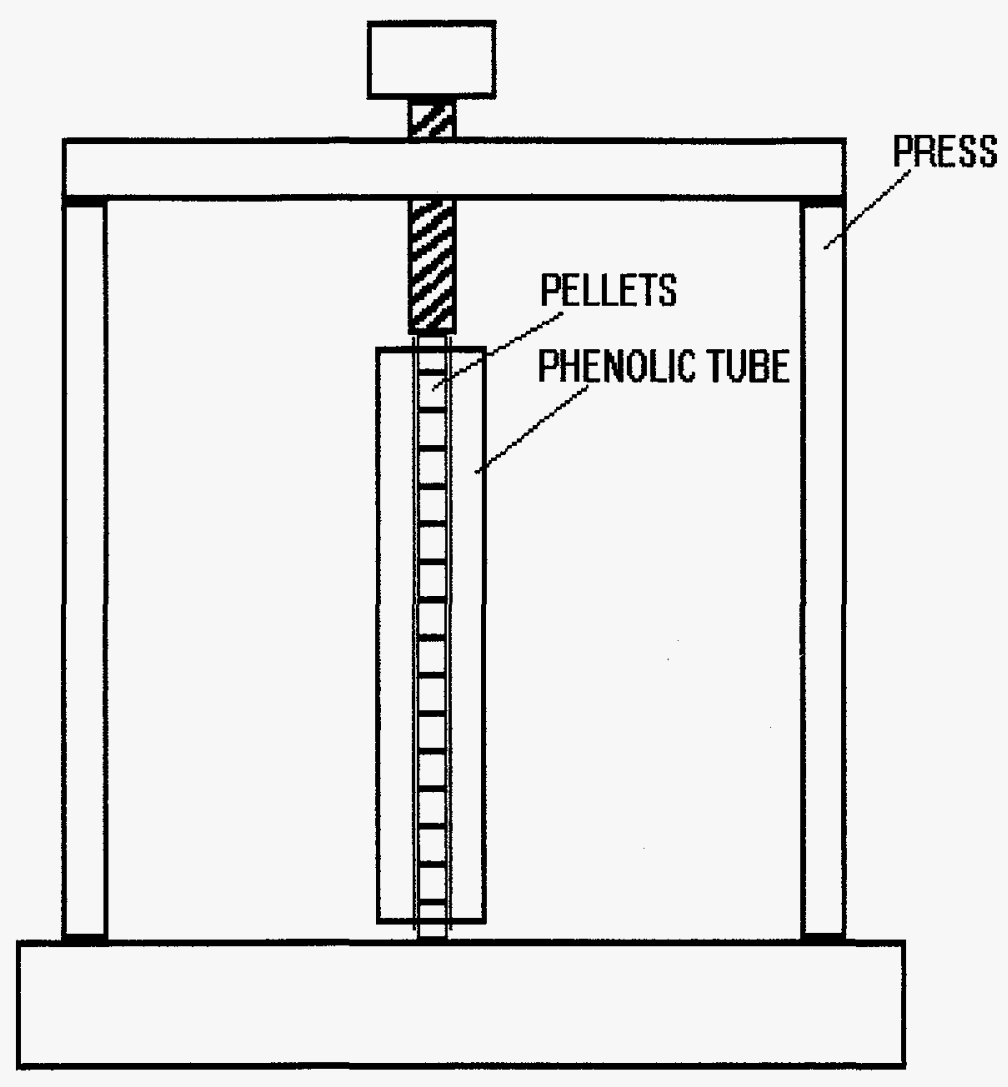

Figure D.1 Explosive fabrication setup.

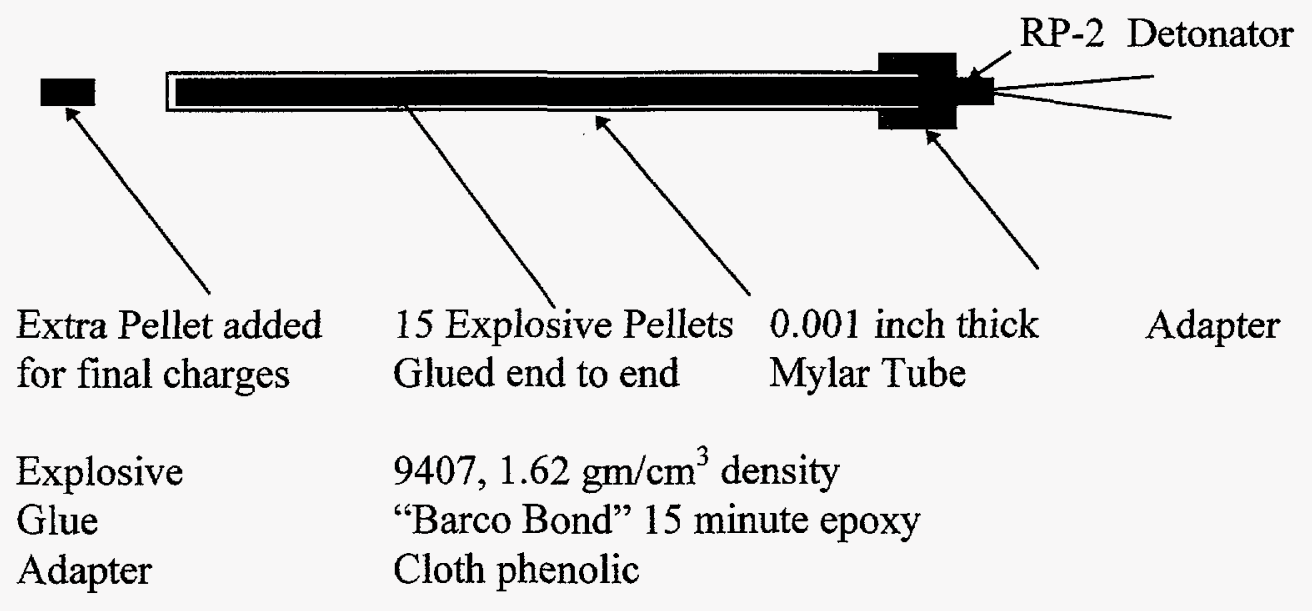

Figure D.2. Sketch of explosive system. 
Figure D.3. Explosive system minus the detonator, mounted on the support pedestal.

\section{D.3 The Explosive System Checkout Test}

To demonstrate the performance of the explosive system, one charge was built and attached to the pedestal. The entire system was centered inside a tube (1.5 inch OD and $\sim 20$ inch long). The tube had five layers of 0.25 inch thick, hard rubber wrapped around the outside of the tube. The rubber was needed to back-up the carbon gage to simulate the dirt packing in the centrifuge test (so that a representative pressure pulse would be obtained). Figures D.4 and D.5 show the test configuration.

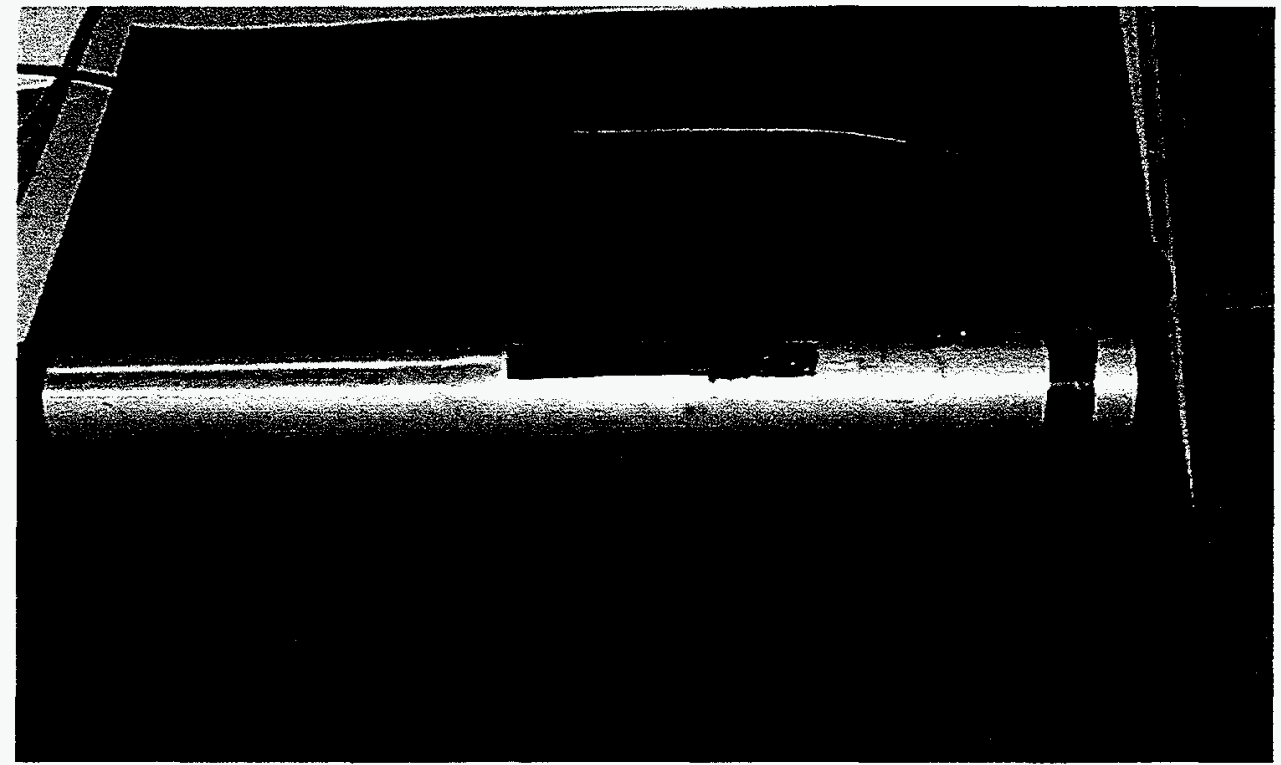

Figure D.4. Tube with the thin film carbon gage in place (electrical tape was placed over the gage). 


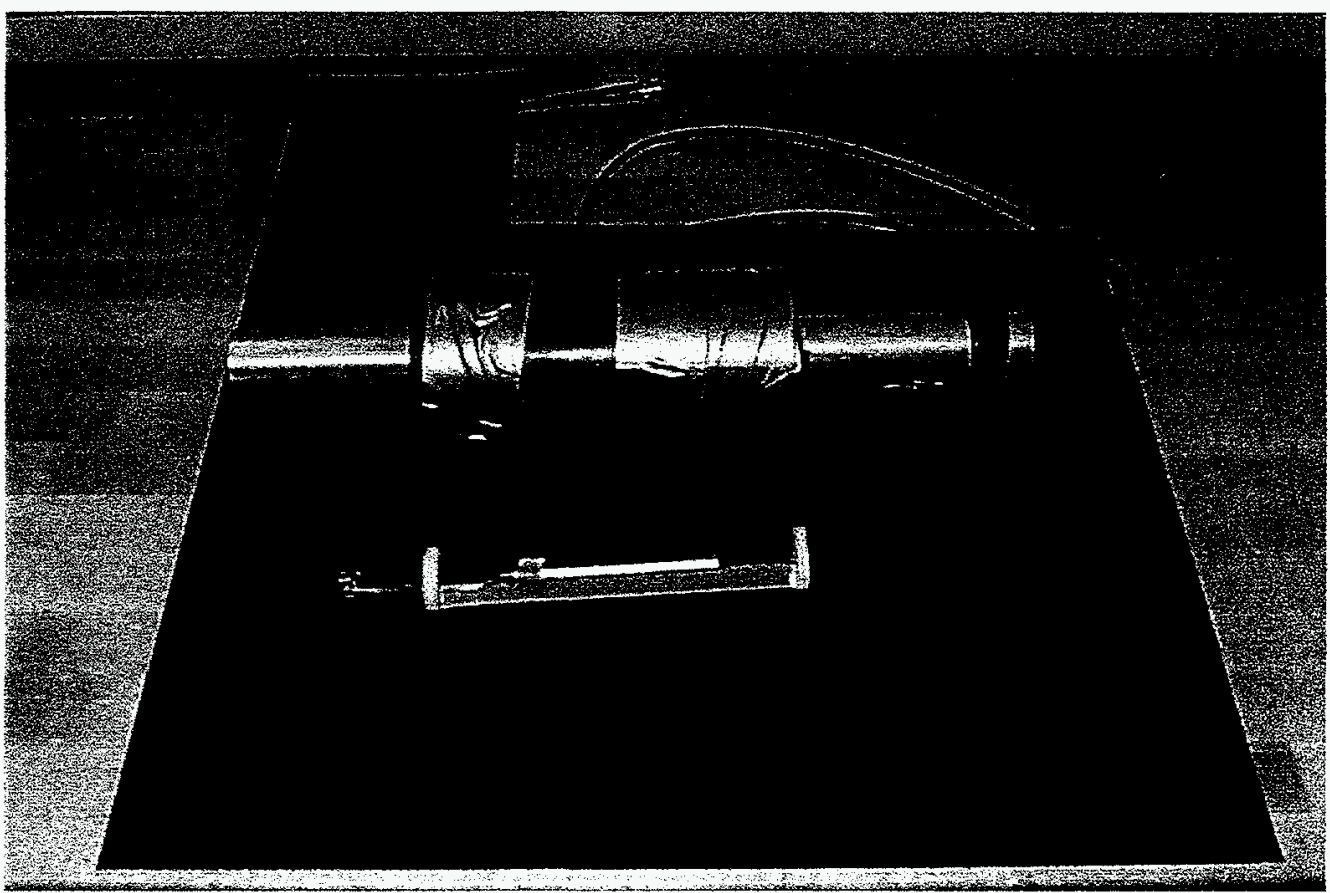

Figure D.5. Explosive charge on the pedestal with the rubber covered tube.

\section{D.4 The Instrumentation used in the Explosive System Checkout Test}

A thin film carbon stress gage was mounted on the pipe to measure the pressure profile at the interface of the pipe and the rubber sheet that was wrapped around the pipe. The carbon gage is comprised of a small ( $0.05 \times 0.05$ inch) graphite element electrically connected to copper leads and encapsulated in Kapton. The element thickness is 0.0005 inch. The sensitive element of the gage was epoxied to the pipe using a fast bonding cyanoacrylate epoxy (910). The remainder of the Kapton with the integral leads was left unbonded. Four pieces of electrical tape were placed between the lead attachment point on the carbon gage and the pipe as a means of mitigating stresses on the gage leads. Magnet wire (38 gage) was attached to the exposed copper tabs to reduce spallation and handling problems associated with large lead attachments. The leads were tightly twisted (to reject electrical "noise" pickup from the fire set discharge) and approximately 4 inches long. The magnet wire was attached directly to a 4 foot long RG-58 C/U coaxial cable. The entire gage assembly was then covered with a piece of electrical tape. A sheet of 0.25 inch thick rubber was wrapped tightly around the pipe (five layers thick) in the loaded area as a simulation of the earth's mechanical impedance.

The carbon gage was conditioned by a pulsed power supply (PPS) which contained circuitry for $50 \mathrm{ohm}$ bridge completion, bridge balancing, triggering and a battery powered excitation source ( 2 each, 30 volt batteries placed in series). The output of the PPS was connected to a Digital Storage Oscilloscope (DSO) with an RG-58 C/U instrumentation cable. The DSO was operated 
in the high input impedance mode. The sample interval on the DSO was $8 \mathrm{~ns} / \mathrm{pt}$. The vertical sensitivity on the DSO was 0.08 volt/division and the trace was offset to the bottom of the CRT.

The carbon gage used for this experiment is very small and heating of the element due to current flow causes a baseline shift (temperature induced resistance change) of the transducer. To compensate for this baseline shift, the PPS was triggered after the test setup was in place and the resulting baseline shift was recorded without the explosive being detonated. This data was then subtracted from the data obtained during the actual explosive test to compensate for the PPS temperature effects.

\section{D.5 The Initiation System used in the Explosive System Checkout Test}

The RP-2 detonator used in this experiment was initiated by a TC1061 fireset using a TC1062 voltage divider/current viewing assembly. This fireset was chosen because of its' low noise characteristics and its' proven performance in component testing. This resulted in data being obtained that was free from fireset interference at the time of pressure response.

\section{D.6 The Explosive System Checkout Test Results}

Figure D.6 shows the experimental setup inside the test chamber before the test. Figure D.7 shows the material after the test. Figure D.8 shows the bulge in the tube after the test.

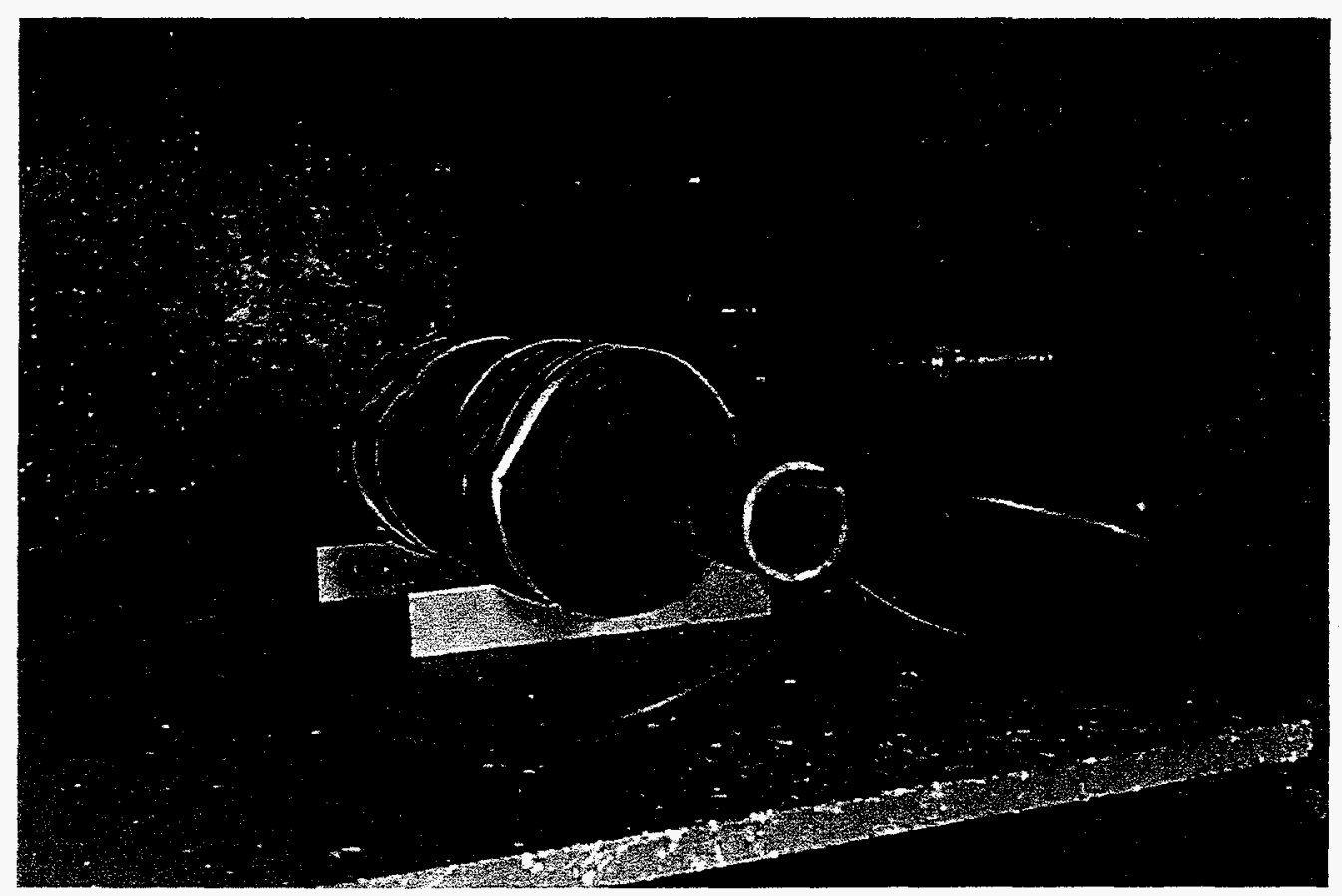

Figure D.6. Test Setup In Test Chamber, Pre-Test. 


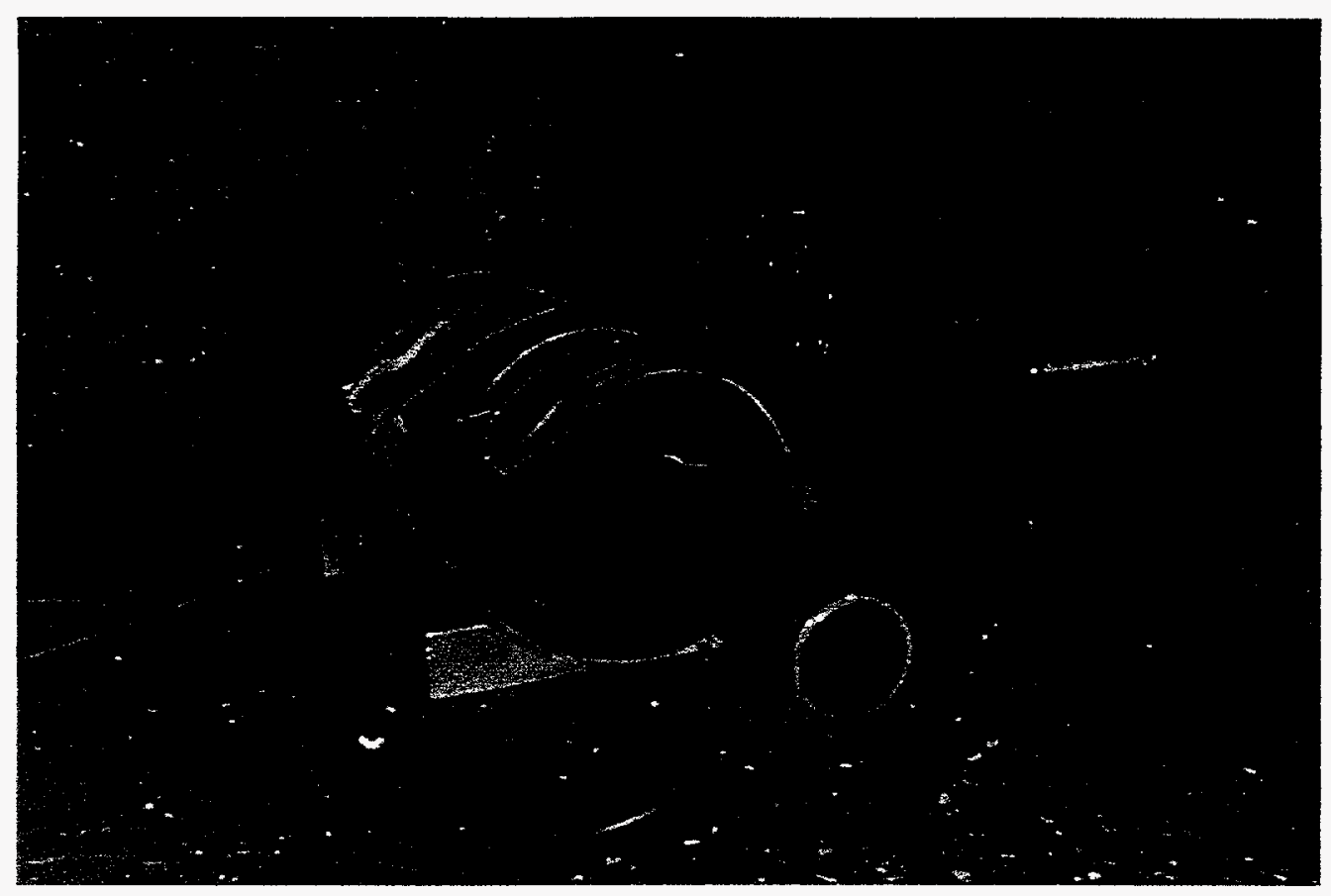

Figure D.8. Test Setup In Test Chamber, Post-Test.

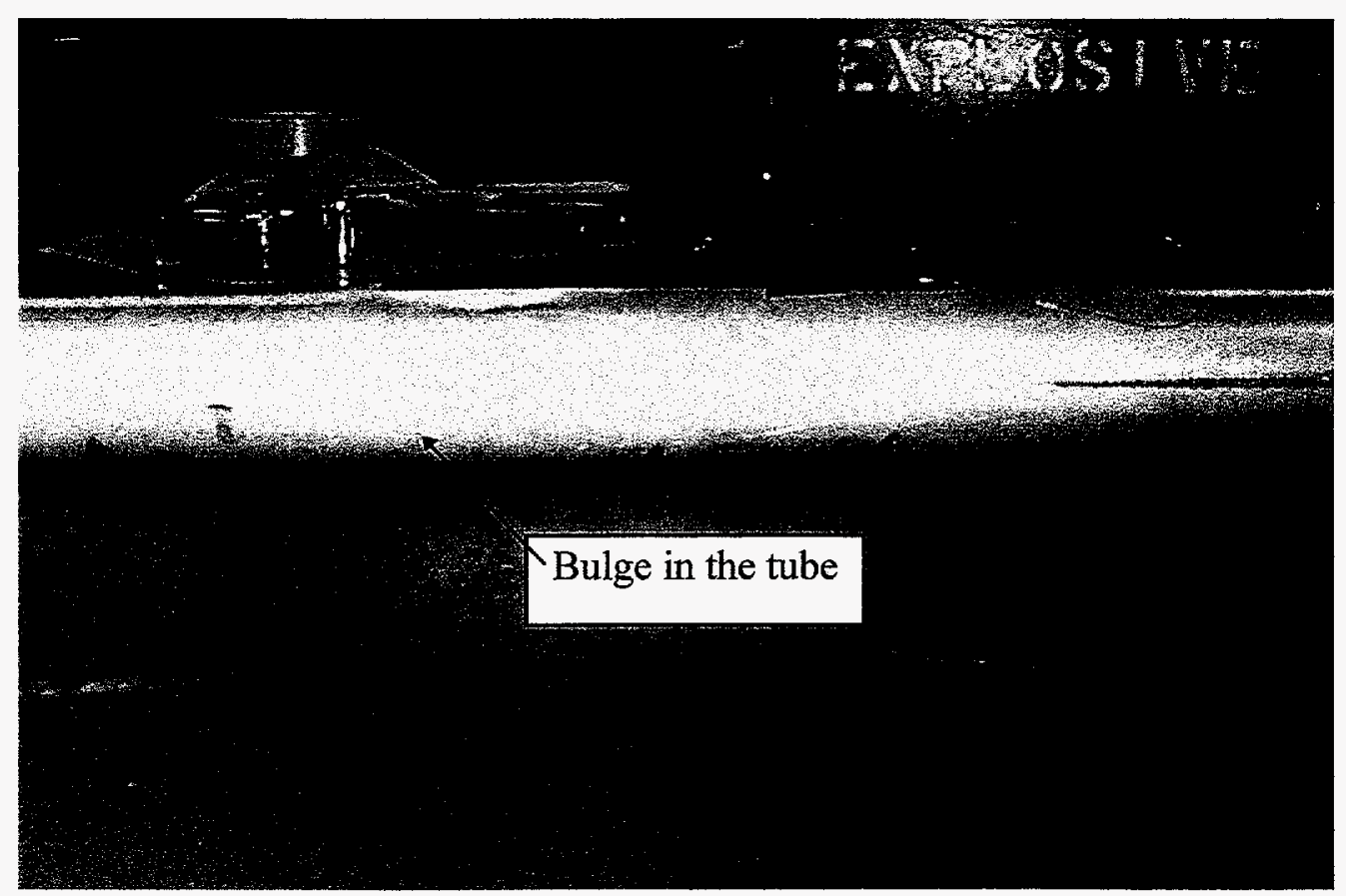

Figure D.8. Tube, showing the bulge after the test.

Figure D.9 shows the explosive test data superimposed on the pre-test gage heating data. The baseline shift during the explosive test matches the pre-test heating data. Figure D.10 is the 
pressure data in engineering units (minus the pre-test heating) with the integration of this data superposed on the same output history. The pressure pulse recorded from this test shows a peak of 13,600 psi. The integration of the pressure gives the impulse imparted to the tube wall, $0.067 \mathrm{psi}$-s. The enveloping design calculation yielded an impulse of $0.084 \mathrm{psi}$-s, slightly greater than measured but within the expected range.

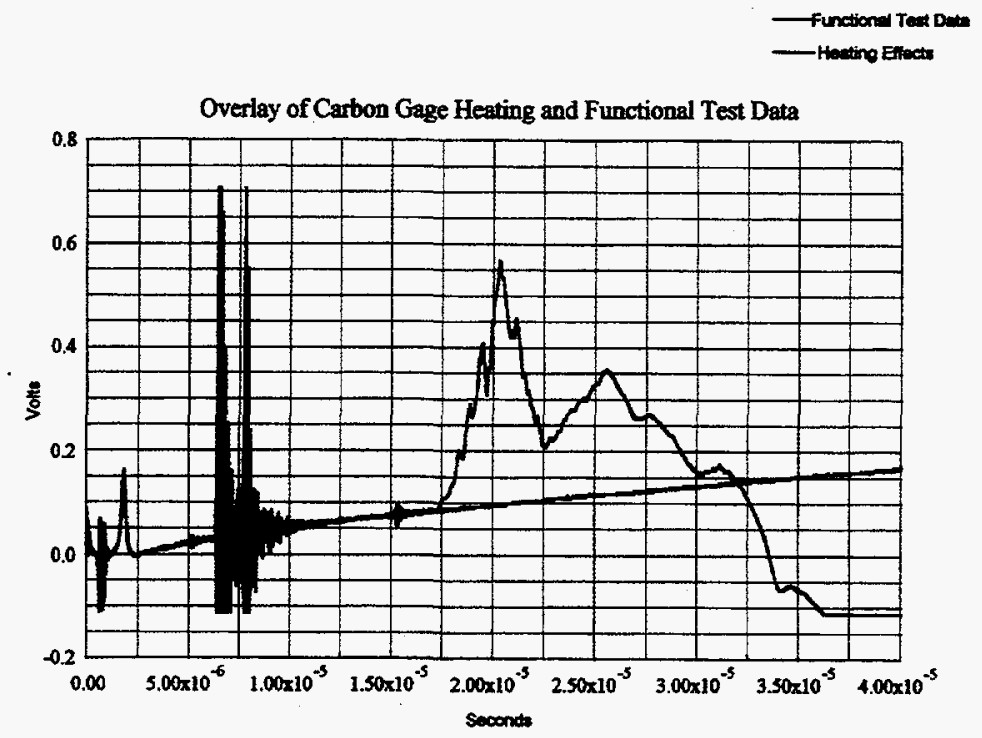

Figure D.9. Data from pretest heating superimposed on the explosive test data.

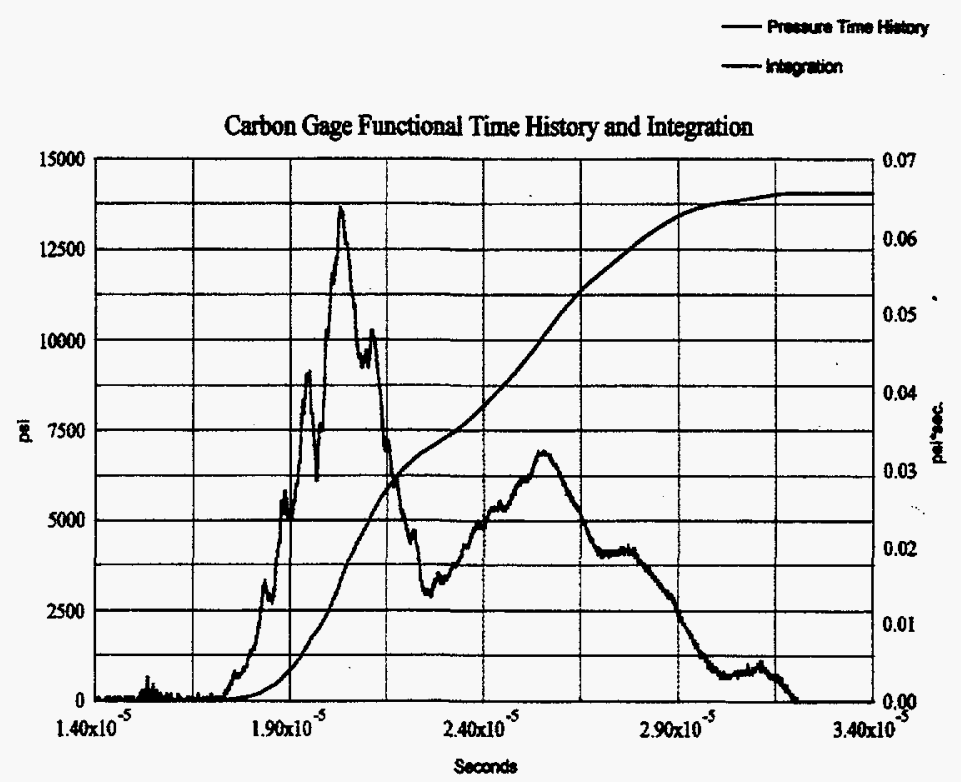

Figure D.10. Corrected Data from the Explosive Test along with the Integrated Data. 
This page intentionally left blank. 


\section{References}

Budge, K.G., Peery, J.S., Robinson, A. C., Summers, R. M., and Wong, M.K., "ALEGRA: User Input and Physics Descriptions," In Preparation, Sandia National Laboratories, 1997.

Budge, K. G., Peery, J.S. Trucano, T.G. and Wong, M. K., "ALEGRA: Verification Test Suite," In Preparation, Sandia National Laboratories, 1997.

Gaffney, E. S., "Containment Science on a Centrifuge," Proceedings of the 2nd Symposium on Containment of Underground Nuclear Explosives," CONF-830882, Volume 12, pp. 365-378, Kirtland Air Force Base, Albuquerque, New Mexico, August 2-4, 1983.

Popov, E., P., Engineering Mechanics of Solids, Prentice-Hall, 1990.

Mosaic, "What's Fail-safe for a Dam," National Science Foundation, Volume 10, Number 4, July/August 979.

Schmidt, R. M. and Holsapple, K. A., "Theory and Experiments on Centrifuge Cratering," Journal of Geophysical Research, Volume 85, Number B1, pp. 235-252, January 10, 1980.

Tieszen, S. R. and Attaway, S. W., "Fuel Dispersion in High-Speed Aircraft/Soil Impact Scenarios," SAND96-0105, Sandia National Laboratories, January, 1996. 
Distribution:

\begin{tabular}{|c|c|c|c|c|}
\hline $\begin{array}{l}\text { No. of } \\
\text { Copies }\end{array}$ & Mail Stop & Last Name & $\begin{array}{l}\text { First Name \& } \\
\text { Initial }\end{array}$ & Organization \\
\hline 1 & 1453 & Benham, & Robert A. & 01553 \\
\hline 1 & 1453 & Braaten, & Floyd $\mathrm{H}$. & 01553 \\
\hline 1 & 1453 & Duggins, & Benjamin D. & 01553 \\
\hline 1 & 1454 & Bonzon, & Lloyd L. & 01554 \\
\hline 1 & 1454 & Wackerbarth, & David E. & 01554 \\
\hline 1 & 0315 & McDowell, & Jerry L. & 02411 \\
\hline 1 & 0419 & Rogers, & Jonathan D. & 05412 \\
\hline 1 & 0761 & Luetters, & Frederick $\mathrm{O}$. & 05800 \\
\hline 1 & 0761 & Matalucci, & Rudolph V. & 05822 \\
\hline 1 & 0751 & Costin, & Laurence S. & 06117 \\
\hline 1 & 0751 & Preece, & Dale S. & 06117 \\
\hline 1 & 1139 & Blanchat, & Thomas K. & 06423 \\
\hline 1 & 1139 & Reil, & Kenneth $\mathrm{O}$. & 06423 \\
\hline 1 & 0151 & Yonas, & Gerold & 09000 \\
\hline 1 & 0151 & Skocypec, & Russell D. & 09002 \\
\hline 1 & 0828 & Thomas, & Robert K. & 09104 \\
\hline 1 & 0437 & Attaway, & Stephen W. & 09118 \\
\hline 1 & 0819 & Peery, & James S. & 09231 \\
\hline 1 & 0819 & Weatherby, & Joe $R$. & 09231 \\
\hline 1 & 0820 & Yarrington, & Paul & 09232 \\
\hline 1 & 1165 & Polito Jr., & Joseph & 09300 \\
\hline 1 & 1157 & Baca, & Michael L. & 09333-1 \\
\hline 1 & 1156 & Stofleth, & Jerome $\mathrm{H}$. & 09333 \\
\hline 1 & 1156 & Tachau, & Robert & 09333 \\
\hline 1 & 0469 & McCaughey, & Kathleen G. & 09700 \\
\hline 1 & 0557 & Baca, & Thomas J. & 09741 \\
\hline 1 & 0555 & Bateman, & Vesta & 09742 \\
\hline 1 & 0555 & Brown & Fred & 09742 \\
\hline 1 & 0555 & Floyd, & Dennis W. & 09742 \\
\hline 1 & 1135 & Calderone, & James J. & 09761 \\
\hline 10 & 1135 & Davie, & Neil T. & 09761 \\
\hline 5 & 1135 & Garcia, & John R. & 09761 \\
\hline 1 & 1135 & Togami, & Thomas C. & 09761 \\
\hline 1 & 0188 & \multicolumn{2}{|l|}{ LDRD Office } & 04523 \\
\hline 1 & 9018 & \multicolumn{2}{|c|}{ Central Technical Files } & $08940-2$ \\
\hline 2 & 0899 & \multicolumn{2}{|c|}{ Technical Library } & 04916 \\
\hline 2 & 0619 & \multicolumn{2}{|c|}{ Review \& Approval Desk } & 12690 \\
\hline 3 & 0161 & \multicolumn{2}{|c|}{ Patents and Licensing } & 11500 \\
\hline
\end{tabular}

